\title{
Synthesis and Structure Revision of Calyxin Natural Products
}

Xia Tian, James J. Jaber and Scott D. Rychnovsky*

\section{SUPPORTING INFORMATION}

Table of Contents................................................... S1

General Experimental Section.................................. S1

Experimentals..................................................... S2-S10

Tabulated NMR data.................................................. S11-S16

Optical Rotation of Calyxin L................................. S17

Proton and Carbon NMR Spectra.............................. S18-S63

General Experimental. ${ }^{1} \mathrm{H}$ NMR spectra were recorded at $500 \mathrm{MHz}$ and ${ }^{13} \mathrm{C}$ NMR spectra were recorded at $125 \mathrm{MHz}$. Chemical shifts of the ${ }^{1} \mathrm{H}$ NMR spectra were referenced to residual chloroform at $7.26 \mathrm{ppm}$. Chemical shifts of ${ }^{13} \mathrm{C}$ NMR spectra were referenced to $\mathrm{CDCl}_{3}$ at $77.0 \mathrm{ppm}$. Coupling constants ( $J$ values) are reported in $\mathrm{Hz}$. Tetrahydrofuran (THF), $\mathrm{Et}_{2} \mathrm{O}$, and $\mathrm{CH}_{2} \mathrm{Cl}_{2}$ were dried by filtration through alumina. Liquid chromatography was performed using forced flow (flash chromatography) of the indicated solvent system on silica gel. Enantiomeric excess was determined by HPLC utilizing a chiralcel OD-H column. Moisture sensitive reactions were performed under an atmosphere of argon using flame or oven dried glassware, and standard syringe/septa techniques. 
Instrumentation: ${ }^{1} \mathrm{H}$ NMR spectra were recorded at 500 or $400 \mathrm{MHz} .{ }^{13} \mathrm{C}$ NMR spectra were recorded on at 125 or $100 \mathrm{MHz}$. Chemical shifts were recorded as $\delta$ values in ppm and are referenced to residual solvent peaks. Coupling constants are reported in $\mathrm{Hz}$.

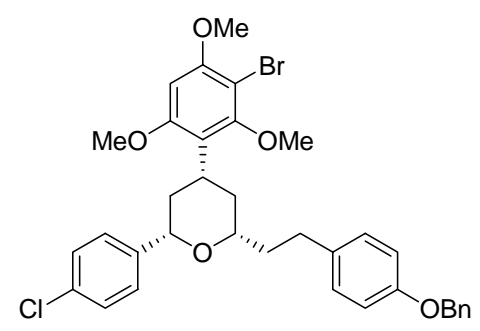

Tetrahydropyran 7: To a cooled solution $\left(0{ }^{\circ} \mathrm{C}\right.$ ) of $\alpha$-acetoxy ether 5 (200 mg, 0.43 mmol, 1 equiv) and bromo-2,4,6-trimethoxybenzene 6 (212 mg, $0.86 \mathrm{mmol}, 2$ equiv) in $\mathrm{CH}_{2} \mathrm{Cl}_{2}(4.5 \mathrm{~mL})$ was added $\mathrm{BF}_{3} \cdot \mathrm{OEt}_{2}(5.5 \mu \mathrm{L}, 0.043 \mathrm{mmol}, 10 \mathrm{~mol} \%)$. The resulting solution was stirred at $0{ }^{\circ} \mathrm{C}$ for $1 \mathrm{~h}$, whereupon saturated aqueous $\mathrm{NaHCO}_{3}$ was added. The reaction mixture was warmed to rt, extracted with $\mathrm{CH}_{2} \mathrm{Cl}_{2}$, washed with brine, dried over anhydrous $\mathrm{MgSO}_{4}$, filtered and concentrated in vacuo. The crude product was purified by MPLC on silica gel (5-10\% EtOAc/hexanes x 2) to afford $167 \mathrm{mg}$ (60\%) of 7 as a white solid: $[\alpha]^{25}{ }_{\mathrm{D}}=-1.7\left(\mathrm{c} 1.0, \mathrm{CH}_{2} \mathrm{Cl}_{2}\right)$; m. p. 47-50 ${ }^{\circ} \mathrm{C}$; IR (thin film) 2930, 2853, 1570, $1509 \mathrm{~cm}^{-1} ;{ }^{1} \mathrm{H}$ NMR (400 MHz, $\left.\mathrm{CDCl}_{3}\right) \delta$ 7.45-7.30 (m, $\left.9 \mathrm{H}\right), 7.12$ (d, $J=8.6 \mathrm{~Hz}$, 2 H), 6.91 (d, $J=8.6$ Hz, 2 H), 6.31 (s, 1 H), 5.04 (s, 2 H), 4.47 (d, J 9.7 Hz, 1 H), 3.98 (s, 3H), 3.88 (s, 6 H), 3.62-3.55 (m, 1 H), 3.54-3.45 (m, 1 H), 2.83-2.70 (m, 2 H), 2.27 (q, $J=12.4 \mathrm{~Hz}, 1 \mathrm{H}), 2.15$ (q, $J=12.4,1 \mathrm{H}), 2.05-2.01(\mathrm{~m}, 1 \mathrm{H}), 1.80-1.76(\mathrm{~m}, 2 \mathrm{H})$, 1.59-1.56 (m, $1 \mathrm{H}) ;{ }^{13} \mathrm{C}$ NMR (100 MHz, $\left.\mathrm{CDCl}_{3}\right) \delta$ 159.0, 156.9, 156.4, 155.6, 141.8, 137.2, 134.6, 132.6, 129.4, 128.5, 128.2, 127.8, 127.4, 127.2, 119.9, 114.6, 98.3, 93.3, 78.7, 77.4, 70.0, 61.7, 56.4, 55.5, 38.0, 37.2, 35.3, 34.1, 30.9; HRMS (ESI) $m$ / $z$ calcd for $\mathrm{C}_{35} \mathrm{H}_{36} \mathrm{BrClO}_{5} \mathrm{Na}[\mathrm{M}+\mathrm{Na}]^{+}$650.1435, found 650.1427. 


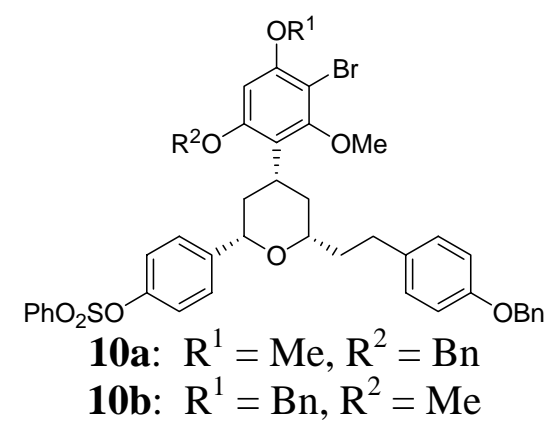

Tetrahydropyran 10a and 10b: To a cooled solution $\left(0^{\circ} \mathrm{C}\right)$ of $\alpha$-acetoxy ether 8 (586 mg, 1.05 mmol, 1 equiv) and bromo-4-benzyloxy-2,6-dimethoxybenzene 9 (500 mg, 2.10 mmol, 2.5 equiv) in $\mathrm{CH}_{2} \mathrm{Cl}_{2}(5 \mathrm{~mL})$ was added $\mathrm{BF}_{3} \cdot \mathrm{OEt}_{2}(13 \mu \mathrm{L}, 0.05 \mathrm{mmol}, 10 \mathrm{~mol} \%)$. The resulting solution was strired at $0{ }^{\circ} \mathrm{C}$ for $30 \mathrm{~min}$, then an additional $10 \% \mathrm{BF}_{3} \cdot \mathrm{OEt}_{2}$ was added. The reaction was allowed to stir at $0{ }^{\circ} \mathrm{C}$ for an additional $1 \mathrm{~h}$, whereupon saturated aqueous $\mathrm{NaHCO}_{3}$ was added. The reaction mixture was warmed to rt, extracted with $\mathrm{CH}_{2} \mathrm{Cl}_{2}$, washed with brine, dried over anhydrous $\mathrm{MgSO}_{4}$, filtered and concentrated in vacuo. The crude product was purified by MPLC (Biotage) on silica gel (15-30\% EtOAc/hexanes) to afford $500 \mathrm{mg}$ (56\%) of a white solid as a 1:2 mixture of 10a and 10b: m. p. 56-59 ${ }^{\circ} \mathrm{C}$; IR (thin film) 3034, 2930 1590, $1509 \mathrm{~cm}^{-1}$; ${ }^{1} \mathrm{H}$ NMR (500 $\left.\mathrm{MHz}, \mathrm{CDCl}_{3}\right) \delta$ 7.87-7.80 (m, $4 \mathrm{H}$ ), 7.67 (dt, $\left.J=7.4,1.4 \mathrm{~Hz}, 2 \mathrm{H}\right), 7.55-7.30(\mathrm{~m}, 26 \mathrm{H}$ ), 7.20 (d, $J=8.6 \mathrm{~Hz}, 2 \mathrm{H}$ ), 7.11 (d, $J=8.6 \mathrm{~Hz}, 2 \mathrm{H}$ ), 7.08 (d, $J=8.6 \mathrm{~Hz}, 2 \mathrm{H}$ ), 6.95-6.86 (m, 8 H), 6.38 (s, 1 H), 6.34 (s, 1 H), 5.15-5.05 (m, 8 H), 4.45-4.39 (m, 2 H), 3.83 (s, 3 H), 3.80 (s, 3 H), 3.79 (s, 3 H), 3.71 (s, 3 H) 3.60-3.45 (m, 4 H), 2.80-2.60 (m, 4 H), 2.302.07 (m, 4 H), 2.00-1.85 (m, 2 H), 1.80-1.65 (m , 4 H), 1.56-1.55 (m, $2 \mathrm{H}) ;{ }^{13} \mathrm{C}$ NMR (125 MHz, $\mathrm{CDCl}_{3}$ ) $\delta$ 159.1, 158.2, 157.2 (2), 156.7 (2), 155.9, 155.1, 148.7 (2), 142.9, 142.6, 137.4, 136.7, 136.6, 135.8, 134.9, 134.8, 134.4 (2), 129.6 (2), 129.4 (3), 129.3, 128.9 (2), 128.8 (4), 128.4, 128.3, 128.1 (2), 127.7 (2), 127.5, 127.3 (3), 122.2 (2), 120.6, 120.5, 114.9 (2), 99.5, 99.0, 95.6, 94.8, 79.1, 78.9, 77.8, 77.3, 71.5, 70.9, 70.3 (2), 62.0 (2), 56.6, 55.8, 38.3 (2), 37.8, 37.4, 35.5, 35.1, 34.4, 34.2, 31.1, 30.8; HRMS (ESI) $\mathrm{m} / \mathrm{z}$ calcd for $\mathrm{C}_{47} \mathrm{H}_{45} \mathrm{BrO}_{8} \mathrm{SNa}[\mathrm{M}+\mathrm{Na}]^{+}$871.1917, found 871.1942. 


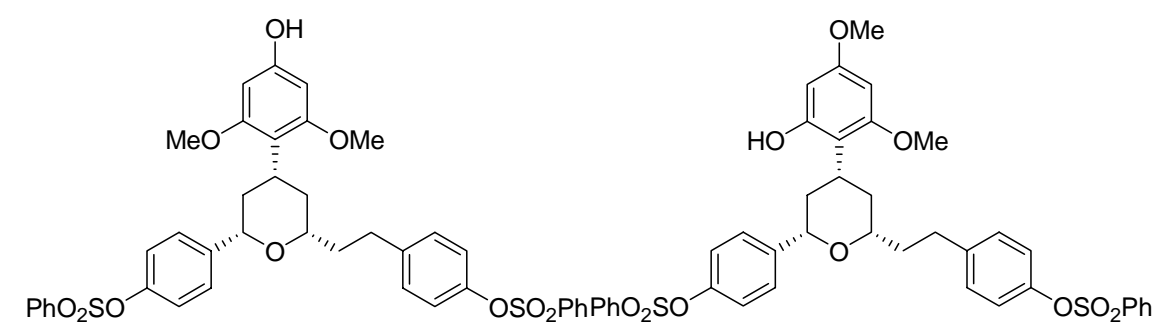

Isomeric prins products (S1, symmetrical) and (S2, unsymmetrical): To a solution of $\alpha$-acetoxy ether $16(1 \mathrm{~g}, 1.57 \mathrm{mmol})$ and arene $9(1 \mathrm{~g}, 3.14 \mathrm{mmol})$ in $3 \mathrm{~mL}$ of dichloromethane at $0{ }^{\circ} \mathrm{C}$ was added $\mathrm{BF}_{3} \cdot \mathrm{OEt}_{2}(0.157 \mathrm{mmol}, 20 \mu \mathrm{L})$ dropwise, upon which the solution darkens, and the bath was removed. Stirring was continued for $3 \mathrm{~h}$ at rt in the dark. The reaction was quenched by dropwise addition of triethylamine until the brownish black color reverted to a light yellow solution. The crude mixture was concentrated in vacuo and purified by flash chromatography (70\% hexanes/25\% dichloromethane/5\% ether) to give the Prins products as a 1:1 mixture (820 mg, 58\%).

The mixture of Prins products ( $400 \mathrm{mg}, 0.445 \mathrm{mmol}$ ) was dissolved in $5 \mathrm{~mL}$ of acetonitrile under Ar, NaI (668 mg, $4.45 \mathrm{mmol}, 10$ equiv.) was added, and it was stirred until homogeneous. The mixture was cooled to $0{ }^{\circ} \mathrm{C}$ and TMS-Cl $(565 \mu \mathrm{L}, 4.45 \mathrm{mmol}$, 10 equiv.) was added dropwise during which time the solution turned reddish brown. The flask was capped with a glass stopper and stirred at $\mathrm{rt}$ for $14 \mathrm{~h}$. The reaction was quenched at $0{ }^{\circ} \mathrm{C}$ with the dropwise addition of saturated aqueous $\mathrm{NaHCO}_{3}$. The mixture was diluted with $3 \mathrm{~mL}$ of brine and $3 \mathrm{~mL}$ of saturated aqueous sodium thiosulfate. The layers were separated and the aqueous layer extracted with $5 \times 10 \mathrm{~mL}$ of methylene chloride and the combined organic layers dried over $\mathrm{MgSO}_{4}$. Filtration followed by concentration in vacuo and purification by column chromatography (35\% EtOAc/hexanes) yielded the two regioisomeric phenols S1 (symmetrical) and S2 (unsymmetrical) in 34\% and 31\% yield: S1 (symmetrical phenol): $[\alpha]{ }^{24}=-9.64$ (c 1.1, $\mathrm{CHCl}_{3}$ ); IR (neat/NaCl) 3486, 2925, 2843, 1600, 1502, 1449, 1372, 1198, $1151 \mathrm{~cm}^{-1} ;{ }^{1} \mathrm{H}$ NMR (500 MHz, $\mathrm{CDCl}_{3}$ ) $\delta$ 7.84-7.81 (m, 4H), 7.67-7.64 (m, 2H), 7.53-7.50 (m, 4H), 7.31 (d, $J=8.7,2 \mathrm{H}$ ), 7.07 (d, $J=8.4,2 \mathrm{H}$ ), 6.92 (d, $J=8.6,2 \mathrm{H}$ ), 6.85 (d, $J=8.5,2 \mathrm{H}$ ), 6.05 (s, 2H), 4.92 (s, 1H), 4.39 (dd, $J=11,2.1,1 \mathrm{H}), 3.73$ (s, 6H), 3.55-3.50 (m, 2H), 2.81-2.65 (m, 2H), 2.17 (q, $J=12.4,1 \mathrm{H}), 2.06$ (q, $J=12.4,1 \mathrm{H}), 1.94-1.89$ (m, 1H), 1.74-1.69 (m, 2H), 1.47 (d, $J=13,1 \mathrm{H}) ;{ }^{13} \mathrm{C}$ NMR $\left(125 \mathrm{MHz}, \mathrm{CDCl}_{3}\right) \delta 159.2,155.3$, 
148.4, 147.6, 142.6, 141.7, 135.52, 135.48, 134.1, 129.6, 129.13, 129.09, 128.51, 128.50, 127.0, 122.1, 121.9, 112.8, 92.3, 87.5, 78.9, 77.5, 55.6, 37.7, 36.8, 34.9, 31.9, 31.2; HRMS (ESI) $m / z$ calcd for $\mathrm{C}_{39} \mathrm{H}_{38} \mathrm{O}_{10} \mathrm{~S}_{2}[\mathrm{M}+\mathrm{Na}]^{+}$753.1804, found 753.1806.

S2 (unsymmetrical phenol): $\left[\alpha{ }^{24}{ }_{\mathrm{D}}=-7.57\right.$ (c 1.4, $\mathrm{CHCl}_{3}$ ); IR (neat/ $\mathrm{NaCl}$ ) 3493, 2923, 2843, 1612, 1502, 1449, 1372, 1198, $1150 \mathrm{~cm}^{-1} ;{ }^{1} \mathrm{H}$ NMR (500 MHz, $\left.\mathrm{CDCl}_{3}\right)$ $\delta 7.83-7.81(\mathrm{~m}, 4 \mathrm{H}), 7.67-7.63(\mathrm{~m}, 2 \mathrm{H}), 7.52-7.48(\mathrm{~m}, 4 \mathrm{H}), 7.31$ (d, $J=8.7,2 \mathrm{H}), 7.07$ (d, $J=8.5,2 \mathrm{H}), 6.91$ (d, $J=8.6,2 \mathrm{H}), 6.85$ (d, $J=8.5,2 \mathrm{H}), 6.06$ (d, $J=2.3,1 \mathrm{H}), 5.92$ (d, $J=2.3,1 \mathrm{H}), 5.18$ (s, 1H), 4.40 (dd, $J=11,2.1,1 \mathrm{H}), 3.75$ (s, 3H), 3.71 (s, 3H), 3.51-3.46 (m, 2H), 2.81-2.65 (m, 2H), 2.23 (q, $J=12.5,1 \mathrm{H}), 2.12$ (q, $J=12.5,1 \mathrm{H}), 1.95-1.88$ (m, 1H), $1.78-1.71(\mathrm{~m}, 2 \mathrm{H}), 1.50(\mathrm{~d}, J=13,1 \mathrm{H}) ;{ }^{13} \mathrm{C}$ NMR (125 $\left.\mathrm{MHz}, \mathrm{CDCl}_{3}\right)$ $\delta 159.5,159.1,155.2,148.4,147.5,142.3,141.6,135.5,135.4,134.2,129.6,129.12,129$. $09,128.5,127.0,122.1,121.9,118.7,111.3,94.2,91.6,78.9,77.5,55.6,55.2,37.6,36.7$, 34.7, 32.0, 31.1; HRMS (ESI) $\mathrm{m} / \mathrm{z}$ calcd for $\mathrm{C}_{39} \mathrm{H}_{38} \mathrm{O}_{10} \mathrm{~S}_{2} \quad[\mathrm{M}+\mathrm{Na}]^{+}$753.1804, found 753.1814. Anal. Calcd. for $\mathrm{C}_{39} \mathrm{H}_{38} \mathrm{O}_{10} \mathrm{~S}_{2}$ : C, 64.09; H, 5.24. Found: C, 63.91; H, 5.12.

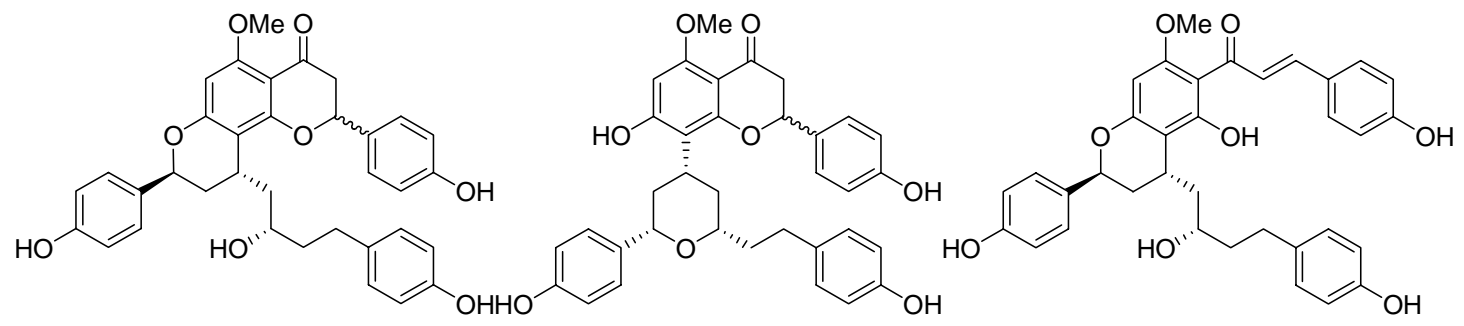

Calyxin G, epicalyxin G, calyxin F, calyxin M, epicalyxin M: Calyxin L (35 mg, $0.060 \mathrm{mmol}$ ) was dissolved in $2 \mathrm{~mL}$ of acetic acid (distilled from $\mathrm{CrO}_{3}$ ) and heated to 90 ${ }^{\circ} \mathrm{C}$ for $14 \mathrm{~h}$. The orange colored solution was allowed to cool then concentrated. The crude mixture was purified by prep-TLC (10\% 9:1 MeOH:acetic acid, 90\% dichloromethane) to give three major products plus starting material. Further purification on reverse phase HPLC (Phenomenex Luna c-18 column; 60:40 acetonitrile:water with $0.1 \%$ TFA) gave the purified final products:

Recovered starting material (6 mg, 17\%)

Calyxin G/epicalyxin G (7 mg, 20\%): $\quad$ IR (neat/NaCl) 3391, 1604, 1516, 1155; ${ }^{1} \mathrm{H}$ NMR (500 MHz, CD $\left.{ }_{3} \mathrm{OD}\right) \delta 7.34$ (dd, $\left.J=8.5,5,2 \mathrm{H}\right), 7.23$ (d, $\left.J=8.5,2 \mathrm{H}\right), 6.88-6.77$ 
(m, $6 \mathrm{H}$ ), 6.65 (d, $J=8.5,2 \mathrm{H}), 6.13$ (s, 1H), 5.32 (d, $J=13.5,1 \mathrm{H}), 5.05$ (d, $J=11.5,2.0$, 1H), 3.78 (s, 3H), 3.57-3.52 (m, 1H), 3.08 (dd, $J=13.9,7.7,0.5 \mathrm{H}), 3.04$ (dd, $J=13.9$, 7.7, 0.5H), 3.01-2.91 (m. 1H), 2.64 (t, $J=18,1 \mathrm{H}), 2.55-2.49$ (m, 0.5H), 2.42-2.36 (m, 1H), 2.26-2.32 (m, 0.5H), 2.17 (dd, $J=13.6,1.2,1 \mathrm{H}), 1.99-1.92$ (m, 0.5H), 1.89-1.79 (m, 1.5H), 1.67-142 (m, 2H); ${ }^{13} \mathrm{C}$ NMR (125 MHz, CD $\left.{ }_{3} \mathrm{OD}\right) \delta 192.74,192.71,163.8$, 163.7, 163.3, 163.1, 161.8, 161.7, 159.24, 159.17, 158.5, 156.2, 134.4, 133.2, 133.17, $131.1,131.0,130.3,129.3,129.2$, 128.7, 116.5, 116.2, 116.06, 116.05, 108.9, 108.8, 106.6, 106.2, 94.8, 94.6, 80.73, 80.71, 75.9, 75.8, 72.1, 71.8, 56.2, 46.21, 46.15, 44.0, 43.8, 40.8, 40.7, 35.1, 34.9, 32.09, 32.06, 29.7, 29.6; HRMS (ELS) $\mathrm{m} / \mathrm{z}$ calcd. for [M + $\mathrm{Na}]^{+}$605.2151, found 605.2137.

Calyxin F (3.7 mg, 11\%): $[\alpha]_{\mathrm{D}}^{24}=+16.3$ (c 0.175, MeOH); IR (neat/ $\left.\mathrm{NaCl}\right)$ 3349, 2361, 1605, 1514, 1226, 1153; ${ }^{1} \mathrm{H}$ NMR (500 MHz, CD $\left.{ }_{3} \mathrm{OD}\right) \delta 7.83$ (d, $\left.J=15.5,1 \mathrm{H}\right), 7.72$ (d, $J=15.5,1 \mathrm{H}), 7.51$ (d, $J=8.5,2 \mathrm{H}), 7.25$ (d, $J=8.5,2 \mathrm{H}), 7.02$ (d, $J=8.5,2 \mathrm{H}), 6.83$ (d, $J$ $=8.5,2 \mathrm{H}), 6.79$ (d, $J=8.5,2 \mathrm{H}), 6.68$ (d, $J=8.5,2 \mathrm{H}), 6.03$ (s, 1H), 5.12 (d, $J=11.7$, $1 \mathrm{H}), 3.90$ (s, 3H), 3.81-3.74 (m, 1H), 3.13-3.11 (m, 1H), 2.74 (ddd, $J=18,10,5.5,1 \mathrm{H})$, 2.59 (ddd, $J=18,9.5,6.5,1 \mathrm{H}$ ), 2.20 (d, $J=14,1 \mathrm{H}$ ), 2.02 (dt, $J=14,5,1 \mathrm{H}$ ), 1.94-1.81 (m, 2H), 1.74-1.64 (m, 2H); ${ }^{13} \mathrm{C}$ NMR (125 MHz, CD $\left.{ }_{3} \mathrm{OD}\right) \delta$ 194.2, 166.2, 162.8, 162.4, 161.2, 158.5, 156.3, 144.0, 134.6, 133.4, 131.4, 130.4, 128.7, 128.4, 125.5, 116.9, 116.2, 116.1, 108.2, 106.8, 92.8, 75.9, 71.4, 56.3, 43.6, 40.8, 35.4, 32.0, 28.7; HRMS (ELS) $\mathrm{m} / \mathrm{z}$ calcd. for $[\mathrm{M}+\mathrm{Na}]^{+}$605.2151, found 605.2134.

Calyxin M/epicalyxin M (4 mg, 11\%): IR (neat/ $\mathrm{NaCl}$ ) 3434, 2360, 2342, 1596, 1515, 1218; ${ }^{1} \mathrm{H}$ NMR (500 MHz, CD $\mathrm{CDD}_{3} \delta 7.38$ (d, $\left.J=8.2,2 \mathrm{H}\right), 7.13$ (d, $\left.J=8.5,1 \mathrm{H}\right), 7.05$ (d, $J=8.4,1 \mathrm{H}), 6.98$ (d, $J=8.5,1 \mathrm{H}), 6.96$ (d, $J=8.6,1 \mathrm{H}), 6.87-6.85$ (m, 2H), 6.73 (d, $J$ = 8.5, 1H), 6.69-6.65 (m, 3H), $6.13(\mathrm{~s}, 0.6 \mathrm{H}), 6.12$ (s, 0.4H), 5.33 (dd, $J=13,2.5,0.5 \mathrm{H})$, 5.28 (dd, $J=13,2.5,0.5 \mathrm{H}), 4.27$ (dd, $J=11,1.2,1 \mathrm{H}), 3.79$ (s, 3H), 3.57-3.47 (m, 2H), 3.00 (dd, $J=13.5,12.5,0.5 \mathrm{H}$ ), 2.98 (dd, $J=13.5,12.5,0.5 \mathrm{H}$ ), 2.71 (dd, $J=5,3,0.5 \mathrm{H}$ ), 2.69 (dd, $J=5,3,0.5 \mathrm{H}), 2.62-2.55(\mathrm{~m}, 2 \mathrm{H}), 2.45$ (q, $J=12,0.6 \mathrm{H}), 2.36$ (q, $J=12$, 0.6H), 2.16 (q, $J=12,0.9 \mathrm{H}), 2.07$ (q, $J=12,0.7 \mathrm{H}), 1.78-1.67$ (m, 2.3H), 1.58-1.47 (m, 2.8H); ${ }^{13} \mathrm{C}$ NMR (125 MHz, CD $\left.{ }_{3} \mathrm{OD}\right) \delta 193.1,193.0,164.6,164.4,162.01,161.97$, 159.0, 158.9, 157.8, 157.7, 156.3, 135.7, 135.6, 134.59, 134.57, 131.5, 131.3, 130.4, 
130.3, 129.0, 128.9, 128.8, 116.4, 116.04, 116.02, 115.9, 115.8, 112.8, 112.7, 106.4, 106.2, 93.9, 93.8, 81.6, 80.3, 80.1, 79.1, 56.0, 46.2, 39.6, 39.5, 37.9, 37.6, 35.8, 35.6, 33.5, 33.4, 31.8, 31.7; HRMS (ELS) $m / z$ calcd. for $[\mathrm{M}+\mathrm{Na}]^{+} 605.2151$, found, 605.2156 . $^{2}$

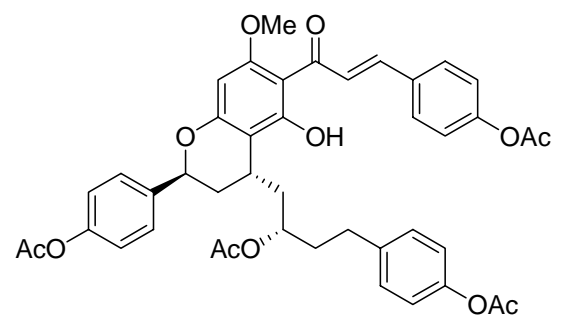

Tetraacetate S3: Calyxin F (0.5 mg, 0.86 umol) was dissolved in $0.5 \mathrm{~mL}$ of pyridine and cooled to $0{ }^{\circ} \mathrm{C}$ and acetic anhydride $(0.5 \mathrm{~mL})$ added. The bath was removed and the mixture stirred for $2 \mathrm{~h}$ at rt. The mixture was then concentrated and purified directly by prep-TLC in 40\% EtOAc/Hexanes to give the tetraacetate (0.5 mg, 77\%): ${ }^{1} \mathrm{H}$ NMR (500 $\left.\mathrm{MHz}, \mathrm{C}_{6} \mathrm{D}_{6}\right) \delta 8.01$ (d, $\left.J=13,1 \mathrm{H}\right), 7.98$ (d, $\left.J=13,1 \mathrm{H}\right), 7.57$ (d, $\left.J=7,2 \mathrm{H}\right), 7.29-7.26$ (m, 4H), 7.06 (d, $J=7,2 \mathrm{H}), 7.01$ (d, $J=7,2 \mathrm{H}), 6.97$ (d, $J=7,2 \mathrm{H}), 5.99$ (s, 1H), 5.28 (d, $J=8.9,1 \mathrm{H}$ ), 5.07 (dddd, $J=8,8,3,3,1 \mathrm{H}$ ), 3.22 (s, 3H), 3.16-3.12 (m, 1H), 2.77 (ddd, $J$ = 12, 8, 4.5, 1H), 2.64-2.59 (m, 1H), 2.35-2.32 (m, 1H), 2.26-2.23 (m, 1H), 2.15-2.09 (m, 1H), 2.06-2.00 (m, 1H), 1.87 (ddd, $J=3,9,13,1 \mathrm{H}), 1.76$ (s, 3H), 1.75 (s, 3H), 1.72 (s, 3H), 1.71 (s, 3H), 1.69-1.68 (m, 1H); HRMS (ELS) m/z calcd. for $\mathrm{C}_{43} \mathrm{H}_{42} \mathrm{O}_{12}[\mathrm{M}+$ $\mathrm{Na}]^{+}$751.2755, found 751.2733.

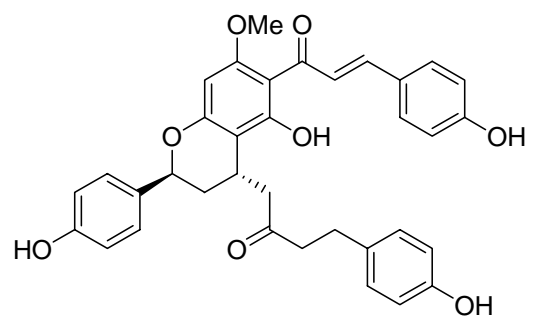

Ketone 23: Calyxin F (1 mg, 1.7 umol) was dissolved in $100 \mathrm{uL}$ of DMSO and DIPEA (12 uL, 40 equiv.) was added. To this mixture was added $20 \mathrm{uL}$ (20 equiv.) of a $1.7 \mathrm{M}$ solution of $\mathrm{SO}_{3}$.pyridine in DMSO. Stirring was continued for $2 \mathrm{~h}$, at which point the mixture was diluted with EtOAc. The organic layer was washed with $1 \mathrm{M} \mathrm{NaHSO}_{4}$ and 
dried with sodium sulfate. Filtration, concentration under reduced pressure followed by purification by prep-TLC (1\% AcOH/9\% $\mathrm{MeOH} / 90 \% \mathrm{CH}_{2} \mathrm{Cl}_{2}$ ) yielded $0.7 \mathrm{mg}$ (70\%) of ketone 23 as a yellow film: ${ }^{1} \mathrm{H}$ NMR (500 MHz, $\left.\mathrm{CD}_{3} \mathrm{OD}\right) \delta 7.83(\mathrm{~d}, J=15.5,1 \mathrm{H}), 7.73$ (d, $J=15.5,1 \mathrm{H}), 7.52$ (d, $J=8.5,2 \mathrm{H}), 7.21$ (d, $J=8.5,2 \mathrm{H}), 7.02$ (d, $J=8.5,2 \mathrm{H}), 6.84$ (d, $J=8.5,2 \mathrm{H}), 6.81$ (d, $J=8.5,2 \mathrm{H}), 6.67$ (d, $J=8.5,2 \mathrm{H}), 6.05$ (s, 1H), 4.96 (d, $J=$ 11.6, 1H), 3.91 (s, 3H), 3.51-3.48 (m, 1H), 3.05 (d, $J=16.5,1 \mathrm{H}), 2.86-2.78(\mathrm{~m}, 4 \mathrm{H})$, 2.69 (dd, $J=16.5,10,1 \mathrm{H}), 2.00-1.93(\mathrm{~m}, 1 \mathrm{H}), 1.88-1.84(\mathrm{~m}, 1 \mathrm{H}) ; \quad{ }^{13} \mathrm{C}$ NMR $(125$ $\left.\mathrm{MHz}, \mathrm{CD}_{3} \mathrm{OD}\right) \delta 212.2$, 194.2, 166.2, 163.2, 163.1, 162.6, 161.3, 158.6, 156.7, 144.1, 133.2, 132.9, 131.4, 130.3, 128.8, 128.3, 125.4, 123.6, 116.9, 116.21, 116.19, 106.4, 92.7, 75.6, 56.4, 45.7, 34.9, 30.2, 26.4; HRMS (ELS) $\mathrm{m} / \mathrm{z}$ calcd. for $\mathrm{C}_{35} \mathrm{H}_{32} \mathrm{O}_{8}[\mathrm{M}+\mathrm{Na}]^{+}$ 603.1995, found, 603.1993.

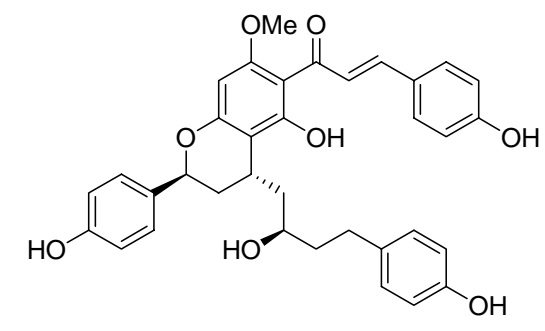

3-epicalyxin F 24: Ketone 23 (1 mg, 1.7 umol) was dissolved in a 1:1 mixture of methanol and dichloromethane $(0.5 \mathrm{~mL}$ total volume $)$ and cooled to $-78{ }^{\circ} \mathrm{C}$. $\mathrm{NaBH}_{4}(2.6$ mg, 40 equiv.) was added at $-78{ }^{\circ} \mathrm{C}$ and the mixture stirred for $2 \mathrm{~h}$. The reaction was quenched at low temperature with $100 \mathrm{uL}$ of acetone and the bath removed. Once the reaction has reached $-10{ }^{\circ} \mathrm{C}$, it was diluted with EtOAc $(5 \mathrm{~mL})$. The organic layer was washed with $1 \mathrm{M} \mathrm{NaHSO}_{4}(5 \mathrm{~mL})$. The layers were mixed thoroughly until the color of the solution changes from orange to yellow. The aqueous layer was extracted three times with $5 \mathrm{~mL}$ EtOAc and the combined organic layers dried over sodium sulfate and concentrated under reduced pressure. Purification by reverse phase HPLC (Phenomenex Luna c-18 column; 1:1 acetonitrile:water with $0.1 \%$ TFA) yielded the two isomeric alcohols, $0.6 \mathrm{mg}$ of 24 (60\%) and $0.2 \mathrm{mg}$ of calyxin F (20\%): Retention time $11.97 \mathrm{~min}$, 3-epicalyxin F; retention time 12.73 min, calyxin F. NMR data for 3-epicalyxin F 23: ${ }^{1} \mathrm{H}$ NMR (500 MHz, CD 3 OD) $\delta 7.86$ (d, $J=15.5,1 \mathrm{H}), 7.74$ (d, $J=15.5,1 \mathrm{H}), 7.55$ (d, $J=$ 
8.5, 2H), 7.23 (d, $J=8.5,2 \mathrm{H}), 7.07$ (d, $J=8.5,2 \mathrm{H}), 6.86$ (d, $J=8.5,2 \mathrm{H}), 6.84$ (d, $J=$ 8.5, 2H), 6.71 (d, $J=8.5,2 \mathrm{H}), 6.08$ (s, 1H), 5.06 (d, $J=11,1 \mathrm{H}), 3.94$ (s, 3H), 3.76-3.56 (m, 1H), 3.49-3.48 (m, 1H), 2.69-2.62 (m, 2H), 2.12 (apparent d, $J=14,1 \mathrm{H}), 2.05$ (ddd, $J=4,9,12,1 \mathrm{H}$ ), 1.89 (ddd, $J=6,11,14,1 \mathrm{H}), 1.83-1.77$ (m, 2H), 1.71 (ddd, $J=2.5,10$, 15, 1H); ${ }^{13} \mathrm{C}$ NMR (125 MHz, CD $\left.3 \mathrm{OD}\right) \delta 194.3,166.2,163.1,162.5,161.3,158.7$, 156.6, 144.1, 134.4, 133.6, 131.5, 130.5, 128.8, 128.6, 125.8, 117.1, 116.4, 116.3, 108.5, 107.1, 92.8, 75.8, 68.9, 56.5, 42.1, 40.9, 34.2, 32.3, 26.9; HRMS (ELS) $m / z$ calcd. for [M $+\mathrm{Na}]^{+}$605.2151, found 605.2144.

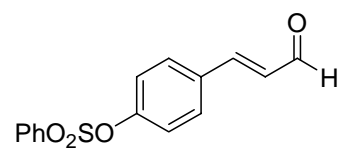

Aldehyde 20: Known methyl (E)-p-hydroxycinnamate ${ }^{1}$ (3 g, $16.8 \mathrm{mmol}$ ) was dissolved in anhydrous acetone $(50 \mathrm{~mL})$ and to this was added $\mathrm{K}_{2} \mathrm{CO}_{3}(2.56 \mathrm{~g}, 18.5 \mathrm{mmol}, 1.1$ equiv.) followed by benzensulfonyl chloride (2.26 mL, $17.6 \mathrm{mmol}, 1.05 \mathrm{mmol})$ and the heterogeneous mixture stirred at room temperature overnight. The solids were filtered and washed with dichloromethane. The combined filtrates was concentrated and then dissolved in $100 \mathrm{~mL}$ of dichloromethane and washed with $1 \mathrm{M} \mathrm{HCl}$ and brine, dried over $\mathrm{MgSO}_{4}$, filtered and concentrated to the crude ester which was used without purification. The crude ester was dissolved in $50 \mathrm{~mL}$ of anhydrous THF and cooled to $-78{ }^{\circ} \mathrm{C}$ at which point DIBAL-H (1 M in toluene, $37 \mathrm{~mL}, 2.2$ equiv.) was added as a steady stream down the side of the flask. The reaction was stirred for $1 \mathrm{~h}$ then placed in an ice for $30 \mathrm{~min}$ and then quenched with $20 \mathrm{~mL}$ of saturated aqueous ammonium chloride and $20 \mathrm{~mL}$ of saturated aqueous sodium potassium tartrate. Once the phases had separated, the aqueous layer was extracted with 3 x $20 \mathrm{~mL}$ of dichloromethane and the combined organics dried over $\mathrm{MgSO}_{4}$. The crude alcohol was used without further purification.

The crude allylic alcohol was dissolved in $50 \mathrm{~mL}$ of reagent grade dichloromethane out of the bottle and $\mathrm{MnO}_{2}$ (7.31 g, $84 \mathrm{mmol}, 5$ equiv.) was added and the heterogeneous mixture stirred for $22 \mathrm{~h}$ at room temperature. The $\mathrm{MnO}_{2}$ was filtered off and washed

\footnotetext{
${ }^{1}$ Pirrung, M. C., Chen, J., Rowley, E. G., McPhail, A. T.; J. Am. Chem. Soc. 1993, 115, 7103-7110
} 
with dichloromethane and the combined filtrate was concentrated to give the crude aldehyde which was passed through a plug of silica gel (15\% $\mathrm{Et}_{2} \mathrm{O} / 15 \% \mathrm{DCM} / 70 \%$ hexanes) to give pure aldehyde 20 as a white solid: m. p. 92-94 ${ }^{\circ} \mathrm{C}$; IR (thin film) 3067, 2824, $1677 \mathrm{~cm}^{-1} ;{ }^{1} \mathrm{H}$ NMR (500 MHz, $\mathrm{CDCl}_{3}$ ) $\delta 9.70$ (d, $\left.J=7.6,1 \mathrm{H}\right), 7.87$ (d, $J=8.3$, 2H), 7.71 (t, $J=7.3,1 \mathrm{H}), 7.56$ (t, $J=7.64,2 \mathrm{H}), 7.51$ (d, $J=8.6,2 \mathrm{H}), 7.42$ (d, $J=16$, $1 \mathrm{H}), 7.08$ (d, $J=8.5,2 \mathrm{H}), 6.66$ (dd, $J=7.6,16,1 \mathrm{H}) ;{ }^{13} \mathrm{C}$ NMR (125 MHz, $\left.\mathrm{CDCl}_{3}\right)$ $\delta$ 193.4, 151.5, 150.8, 135.5, 134.7, 133.2, 130.0, 129.6, 129.5, 128.7, 123.4; HRMS (ELS) $\mathrm{m} / \mathrm{z}$ calcd. for $[\mathrm{M}+\mathrm{Na}]^{+} 311.0354$, found 311.0353 .

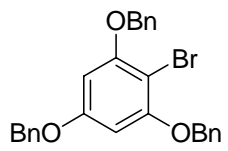

Arene 17: Known tribenzl phloroglucinol (15 g, 37.8 mmol, 1 equiv.) was dissolved in $60 \mathrm{~mL}$ of dichloromethane and cooled to $0{ }^{\circ} \mathrm{C}$. To this was added NBS $(7.4 \mathrm{~g}, 41.7$ mmol, 1.1 equiv.) in $40 \mathrm{~mL}$ of dichloromethane through an addition funnel over $1 \mathrm{~h}$. Upon completion of the addition, the reaction was quenched with $2 \mathrm{~mL}$ of triethylamine and washed with $2 \mathrm{M} \mathrm{NaOH}$, brine, dried over $\mathrm{MgSO}_{4}$, filtered and concentrated. The crude product was recrystallized from $\mathrm{MeOH}$ to give $13.3 \mathrm{~g}$ (74\%) of 17: m. p. 94-96 ${ }^{\circ} \mathrm{C}$; IR (thin film) 3064, 3031, 2359, 1583, 1163, $1115 \mathrm{~cm}^{-1} ;{ }^{1} \mathrm{H}$ NMR (500 MHz, $\mathrm{CDCl}_{3}$ ) $\delta$ 7.47-7.30 (m, $15 \mathrm{H}), 6.29$ (s, 2H), 5.11 (s, 4H), 4.96 (s, 2H); ${ }^{13} \mathrm{C}$ NMR (125 MHz, $\left.\mathrm{CDCl}_{3}\right) \delta 159.5,156.9,136.8,136.6,128.9,128.8,128.4,128.1,127.7,127.2,95.0,94.4$, 71.2, 70.6; HRMS (ELS) $m / z$ calcd. for $[\mathrm{M}+\mathrm{Na}]^{+} 497.0728$, found 497.0733. 


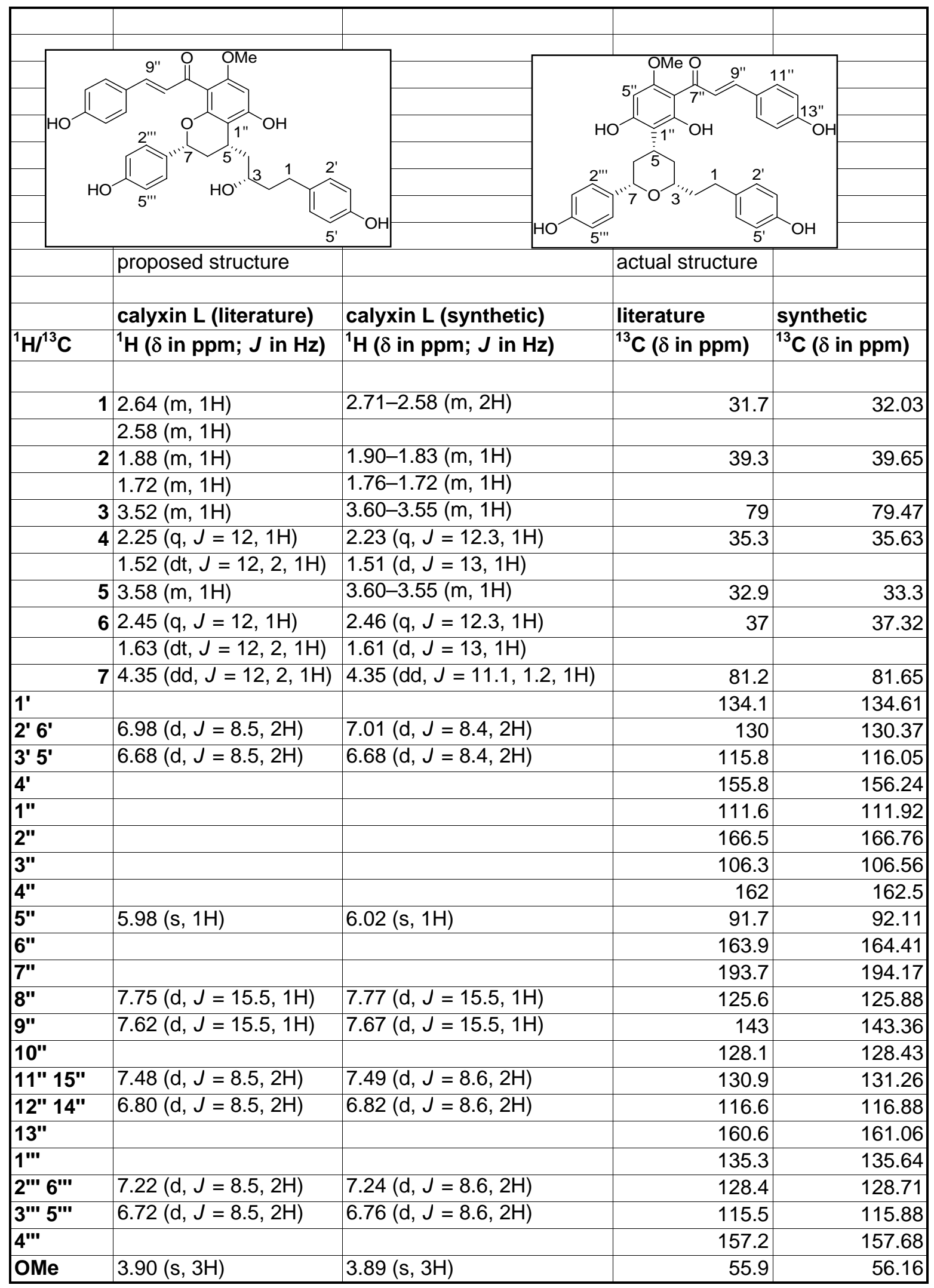




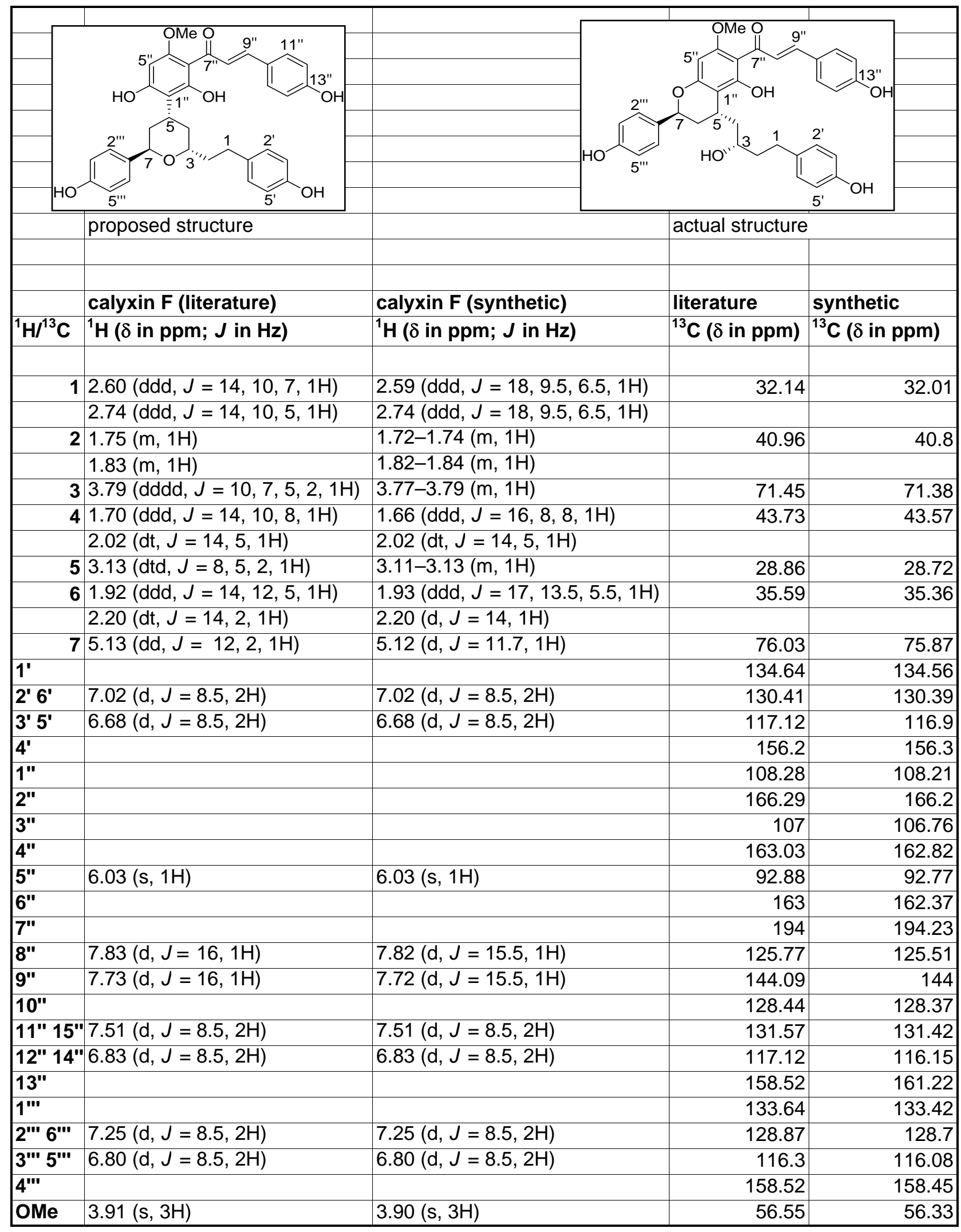




\begin{tabular}{|c|c|c|c|}
\hline & & & \\
\hline & proposed structure & & actual structure \\
\hline & calyxin G (literature) & epi-calyxin G (literature) & G/epi-G mixture (synthetic) \\
\hline${ }^{1} \mathrm{H}$ & ${ }^{1} \mathrm{H}(\delta$ in ppm; $\mathrm{J}$ in $\mathrm{Hz})$ & ${ }^{1} \mathrm{H}(\delta$ in ppm; $\mathrm{J}$ in $\mathrm{Hz})$ & ${ }^{1} \mathbf{H}(\delta$ in ppm; $J$ in $\mathrm{Hz})$ \\
\hline 1 & $2.32(\mathrm{~m}, 1 \mathrm{H})$ & $2.32(\mathrm{~m}, 1 \mathrm{H})$ & $2.42-2.36(\mathrm{~m}, 1 \mathrm{H})$ \\
\hline & $2.41(\mathrm{~m}, 1 \mathrm{H})$ & $2.41(\mathrm{~m}, 1 \mathrm{H})$ & $2.26-2.32(\mathrm{~m}, 0.5 \mathrm{H})$ \\
\hline 2 & $1.46(\mathrm{~m}, 1 \mathrm{H})$ & $1.46(\mathrm{~m}, 1 \mathrm{H})$ & $1.67-1.42(\mathrm{~m}, 2 \mathrm{H})$ \\
\hline & $1.60(\mathrm{~m}, 1 \mathrm{H})$ & $1.60(\mathrm{~m}, 1 \mathrm{H})$ & \\
\hline 3 & $3.56(\mathrm{~m}, 1 \mathrm{H})$ & $3.71(\mathrm{~m}, 1 \mathrm{H})$ & $3.57-3.52(\mathrm{~m}, 1 \mathrm{H})$ \\
\hline 4 & $1.68(\mathrm{ddd}, \mathrm{J}=14,8,6,1 \mathrm{H})$ & $1.68(\mathrm{ddd}, \mathrm{J}=14,8,6,1 \mathrm{H})$ & $1.67-1.42(\mathrm{~m}, 2 \mathrm{H})$ \\
\hline & $1.84(\mathrm{~m}, 1 \mathrm{H})$ & $1.84(\mathrm{~m}, 1 \mathrm{H})$ & \\
\hline 5 & $2.97(\mathrm{~m}, 1 \mathrm{H})$ & $2.97(\mathrm{~m}, 1 \mathrm{H})$ & 3.01-2.91 (m, 1H) \\
\hline 6 & $1.88(\mathrm{td}, \mathrm{J}=12,5,1 \mathrm{H})$ & $1.88(\mathrm{td}, \mathrm{J}=12,5,1 \mathrm{H})$ & $1.89-1.79(\mathrm{~m}, 1.5 \mathrm{H})$ \\
\hline & $2.18(\mathrm{dd}, \mathrm{J}=12,2,1 \mathrm{H})$ & $2.17(\mathrm{dd}, \mathrm{J}=12,2,1 \mathrm{H})$ & $2.17(\mathrm{dd}, \mathrm{J}=13.6,1.2,1 \mathrm{H})$ \\
\hline 7 & $5.07(\mathrm{dd}, \mathrm{J}=12,2,1 \mathrm{H})$ & $5.05(\mathrm{dd}, \mathrm{J}=12,2,1 \mathrm{H})$ & $5.05(\mathrm{dd}, \mathrm{J}=11.5,2.0,1 \mathrm{H})$ \\
\hline 2' 6' & $6.85(\mathrm{~d}, \mathrm{~J}=8.5,2 \mathrm{H})$ & $6.91(\mathrm{~d}, \mathrm{~J}=8.5,2 \mathrm{H})$ & 6.88-6.77 (m, $6 \mathrm{H})$ \\
\hline 3' 5' & $6.66(\mathrm{~d}, \mathrm{~J}=8.5,2 \mathrm{H})$ & $6.66(d, J=8.5,2 H)$ & $6.65(\mathrm{~d}, \mathrm{~J}=8.5,2 \mathrm{H})$ \\
\hline 5" & $6.13(\mathrm{~s}, 1 \mathrm{H})$ & $6.12(\mathrm{~s}, 1 \mathrm{H})$ & $6.13(\mathrm{~s}, 1 \mathrm{H})$ \\
\hline 8" & $2.68(\mathrm{dd}, \mathrm{J}=16.5,3,1 \mathrm{H})$ & $2.76(\mathrm{~d}, \mathrm{~J}=16.5,3.5,1 \mathrm{H})$ & $2.64(\mathrm{t}, \mathrm{J}=18,1 \mathrm{H})$ \\
\hline & $3.05(\mathrm{dd}, \mathrm{J}=16.5,13,1 \mathrm{H})$ & $2.99(\mathrm{dd}, J=16.5,12,1 \mathrm{H})$ & $3.08(\mathrm{dd}, \mathrm{J}=13.9,7.7,0.5 \mathrm{H})$ \\
\hline & & & $3.04(\mathrm{dd}, \mathrm{J}=13.9,7.7,0.5 \mathrm{H})$ \\
\hline & & & 3.01-2.91 (m, 1H) \\
\hline 9" & $5.32(d d, J=13,3,1 H)$ & $5.42(\mathrm{~d}, \mathrm{~J}=12,3.5,1 \mathrm{H})$ & $5.32(\mathrm{~d}, \mathrm{~J}=13.5,1 \mathrm{H})$ \\
\hline 11" 15 & $7.34(\mathrm{~d}, \mathrm{~J}=8.5,2 \mathrm{H})$ & $7.34(\mathrm{~d}, \mathrm{~J}=8.5,2 \mathrm{H})$ & $7.34(\mathrm{dd}, \mathrm{J}=8.5,5,2 \mathrm{H})$ \\
\hline $12 " 14$ & $6.81(\mathrm{~d}, \mathrm{~J}=8.5,2 \mathrm{H})$ & $6.81(\mathrm{~d}, \mathrm{~J}=8.5,2 \mathrm{H})$ & 6.88-6.77 (m, $6 \mathrm{H})$ \\
\hline 2"' 6"' & $7.24(\mathrm{~d}, \mathrm{~J}=8.5,2 \mathrm{H})$ & $7.18(\mathrm{~d}, \mathrm{~J}=8.5,2 \mathrm{H})$ & $7.23(\mathrm{~d}, \mathrm{~J}=8.5,2 \mathrm{H})$ \\
\hline 3"' 5"' & $6.79(\mathrm{~d}, \mathrm{~J}=8.5,2 \mathrm{H})$ & $6.80(\mathrm{~d}, \mathrm{~J}=8.5,2 \mathrm{H})$ & 6.88-6.77 (m, $6 \mathrm{H})$ \\
\hline OMe & $3.79(\mathrm{~s}, 3 \mathrm{H})$ & $3.77(\mathrm{~s}, 3 \mathrm{H})$ & $3.78(\mathrm{~s}, 3 \mathrm{H})$ \\
\hline
\end{tabular}




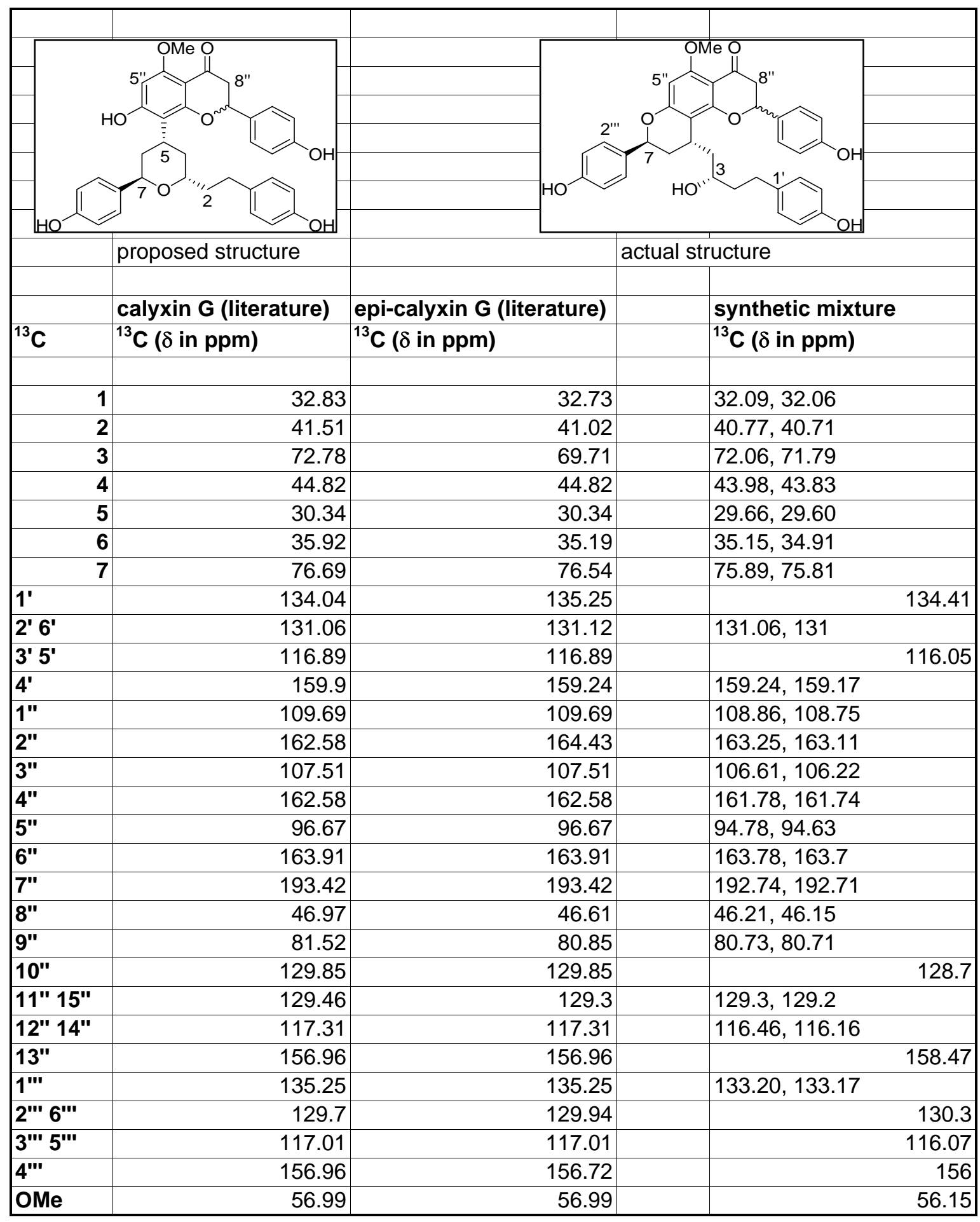




\begin{tabular}{|c|c|c|c|}
\hline & & & \\
\hline & proposed structure & & actual structure \\
\hline & & & \\
\hline & calyxin M (literature) & epi-calyxin M (literature) & M/epi-M mixture (synthetic) \\
\hline${ }^{1} \mathrm{H}$ & ${ }^{1} \mathrm{H}(\delta$ in ppm; $\mathrm{J}$ in $\mathrm{Hz})$ & ${ }^{1} \mathbf{H}(\delta$ in ppm; $\mathbf{J}$ in $\mathbf{H z})$ & ${ }^{1} \mathbf{H}(\delta$ in ppm; $\mathbf{J}$ in $\mathrm{Hz})$ \\
\hline 1 & $2.58(\mathrm{~m}, 2 \mathrm{H})$ & $2.58(\mathrm{~m}, 2 \mathrm{H})$ & $2.62-2.55(\mathrm{~m}, 4 \mathrm{H})$ \\
\hline 2 & $1.76(\mathrm{~m}, 1 \mathrm{H})$ & $1.76(\mathrm{~m}, 1 \mathrm{H})$ & $1.78-1.67(\mathrm{~m}, 4 \mathrm{H})$ \\
\hline & $1.68(\mathrm{~m}, 1 \mathrm{H})$ & $1.68(\mathrm{~m}, 1 \mathrm{H})$ & \\
\hline 3 & $3.88(\mathrm{~m}, 1 \mathrm{H})$ & $3.88(\mathrm{~m}, 1 \mathrm{H})$ & $3.57-3.47(\mathrm{~m}, 2 \mathrm{H})$ \\
\hline 4 & $2.14(q, J=12,1 \mathrm{H})$ & $2.06(q, J=12,1 H)$ & $2.16(q, J=12,1 \mathrm{H})$ \\
\hline & & & $2.07(q, J=12,1 \mathrm{H})$ \\
\hline & $1.52(\mathrm{~m}, 1 \mathrm{H})$ & $1.52(\mathrm{~m}, 1 \mathrm{H})$ & $1.58-1.47(\mathrm{~m}, 2 \mathrm{H})$ \\
\hline 5 & $3.90(\mathrm{~m}, 1 \mathrm{H})$ & $3.90(\mathrm{~m}, 1 \mathrm{H})$ & $3.57-3.47(\mathrm{~m}, 2 \mathrm{H})$ \\
\hline 6 & $2.38(q, J=12,1 H)$ & $2.46(q, J=12,1 H)$ & $2.45(q, J=12,1 \mathrm{H})$ \\
\hline & & & $2.36(q, J=12,1 \mathrm{H})$ \\
\hline & $1.56(\mathrm{dt}, \mathrm{J}=12,2,1 \mathrm{H})$ & $1.56(\mathrm{dt}, \mathrm{J}=12,2,1 \mathrm{H})$ & $1.58-1.47(\mathrm{~m}, 2 \mathrm{H})$ \\
\hline 7 & $4.26(d d, J=12,2,1 H)$ & $4.26(\mathrm{dd}, \mathrm{J}=12,2,1 \mathrm{H})$ & $4.27(\mathrm{dd}, \mathrm{J}=11,1.2,2 \mathrm{H})$ \\
\hline $26^{\prime}$ & $6.96(d, J=8.3,2 \mathrm{H})$ & $6.94(\mathrm{~d}, \mathrm{~J}=8.3,2 \mathrm{H})$ & $6.98(\mathrm{~d}, \mathrm{~J}=8.5,2 \mathrm{H})$ \\
\hline & & & $6.96(d, J=8.6,2 \mathrm{H})$ \\
\hline 3' 5' & $6.68(\mathrm{~d}, \mathrm{~J}=8.3,2 \mathrm{H})$ & $6.66(\mathrm{~d}, \mathrm{~J}=8.3,2 \mathrm{H})$ & $6.69-6.65(\mathrm{~m}, 4 \mathrm{H})$ \\
\hline 5" & $6.11(\mathrm{~s}, 1 \mathrm{H})$ & $6.11(\mathrm{~s}, 1 \mathrm{H})$ & $6.13(\mathrm{~s}, 1.2 \mathrm{H})$ \\
\hline & & & $6.12(\mathrm{~s}, 0.8 \mathrm{H})$ \\
\hline 8" & $2.96(\mathrm{dd}, \mathrm{J}=16.5,12,1 \mathrm{H})$ & $2.98(\mathrm{dd}, \mathrm{J}=16.5,13,1 \mathrm{H})$ & $3.00(\mathrm{dd}, \mathrm{J}=13.5,12.5,1 \mathrm{H})$ \\
\hline & & & $2.98(\mathrm{dd}, \mathrm{J}=13.5,12.5,1 \mathrm{H})$ \\
\hline & $2.65(\mathrm{br} \mathrm{d}, \mathrm{J}=16.5,1 \mathrm{H})$ & $2.68(\mathrm{br} \mathrm{d}, \mathrm{J}=16.5,1 \mathrm{H})$ & $2.71-2.69(\mathrm{~m}, 2 \mathrm{H})$ \\
\hline 9" & $5.26(\mathrm{dd}, J=12,3.5,1 \mathrm{H})$ & $5.30(\mathrm{dd}, \mathrm{J}=13,2.5,1 \mathrm{H})$ & $5.33(\mathrm{dd}, \mathrm{J}=13,2.5,1 \mathrm{H})$ \\
\hline & & & $5.28(\mathrm{dd}, \mathrm{J}=13,2.5,1 \mathrm{H})$ \\
\hline $11 " 15 "$ & $7.35(\mathrm{~d}, \mathrm{~J}=8.5,2 \mathrm{H})$ & $7.35(\mathrm{~d}, \mathrm{~J}=8.5,2 \mathrm{H})$ & $7.38(\mathrm{~d}, \mathrm{~J}=8.2,4 \mathrm{H})$ \\
\hline $12 " 14$ & $6.86(\mathrm{~d}, \mathrm{~J}=8.5,2 \mathrm{H})$ & $6.86(\mathrm{~d}, \mathrm{~J}=8.5,2 \mathrm{H})$ & $6.87-6.85(\mathrm{~m}, 4 \mathrm{H})$ \\
\hline 2"' 6"' & $7.04(\mathrm{~d}, \mathrm{~J}=8.5,2 \mathrm{H})$ & $7.04(\mathrm{~d}, \mathrm{~J}=8.5,2 \mathrm{H})$ & $7.13(\mathrm{~d}, \mathrm{~J}=8.5,2 \mathrm{H})$ \\
\hline & & & $7.05(\mathrm{~d}, \mathrm{~J}=8.4,2 \mathrm{H})$ \\
\hline 3"' 5'" & $6.73(\mathrm{~d}, \mathrm{~J}=8.5,2 \mathrm{H})$ & $6.66(\mathrm{~d}, \mathrm{~J}=8.5,2 \mathrm{H})$ & $6.73(\mathrm{~d}, \mathrm{~J}=8.5,2 \mathrm{H})$ \\
\hline & & & $6.69-6.65(\mathrm{~m}, 2 \mathrm{H})$ \\
\hline OMe & $3.78(\mathrm{~s}, 3 \mathrm{H})$ & $3.78(\mathrm{~s}, 3 \mathrm{H})$ & $3.79(\mathrm{~s}, 6 \mathrm{H})$ \\
\hline
\end{tabular}




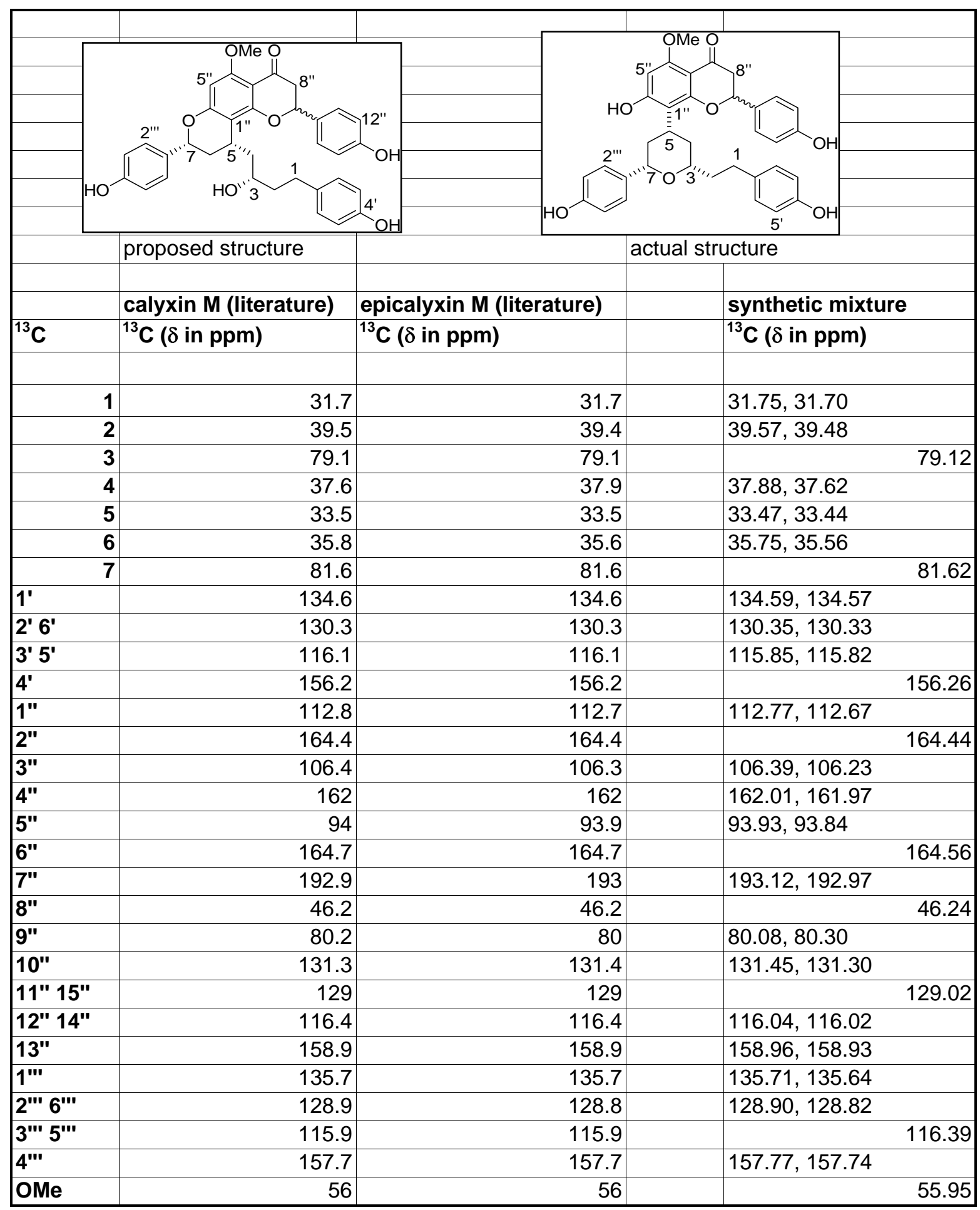




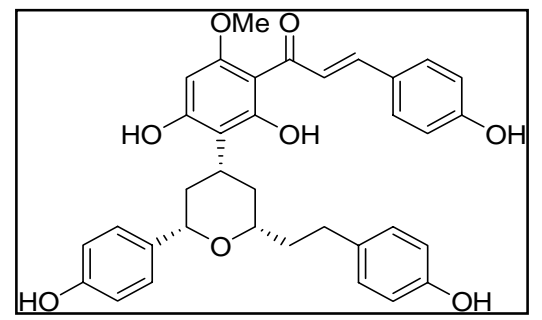

calyxin L

\begin{tabular}{|c|c|c|c|}
\hline \multirow{2}{*}{$\begin{array}{l}\text { Solvent } \\
\text { 1:1 DCM:MeOH }\end{array}$} & \multicolumn{2}{|c|}{$[\alpha]_{D}^{25}$} & 95\% confidence $( \pm)$ \\
\hline & 0.2 & -5 & 2 \\
\hline & 0.08 & -9 & 4 \\
\hline & 0.04 & -19 & 8 \\
\hline & 0.016 & -63 & 25 \\
\hline $\mathrm{MeOH}$ & 0.2 & 0.3 & 2 \\
\hline & 0.08 & -6 & \\
\hline & 0.04 & -5 & 10 \\
\hline & 0.016 & -34 & $1:$ \\
\hline
\end{tabular}


Tian, Jaber, and Rychnovsky

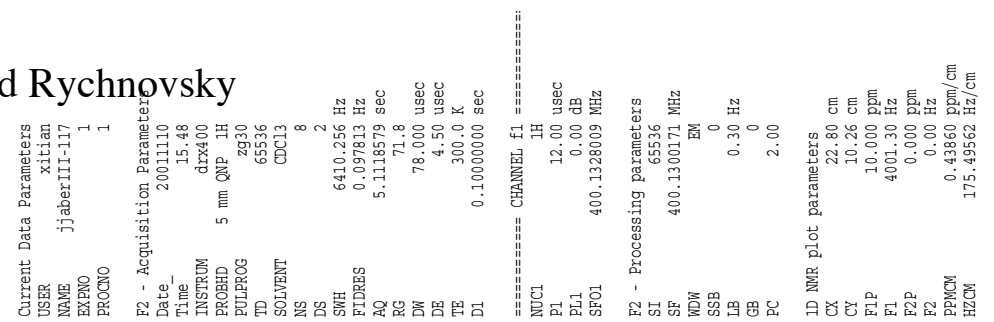

Page S-

18

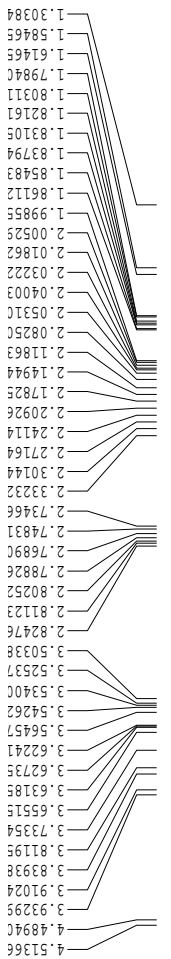

ग
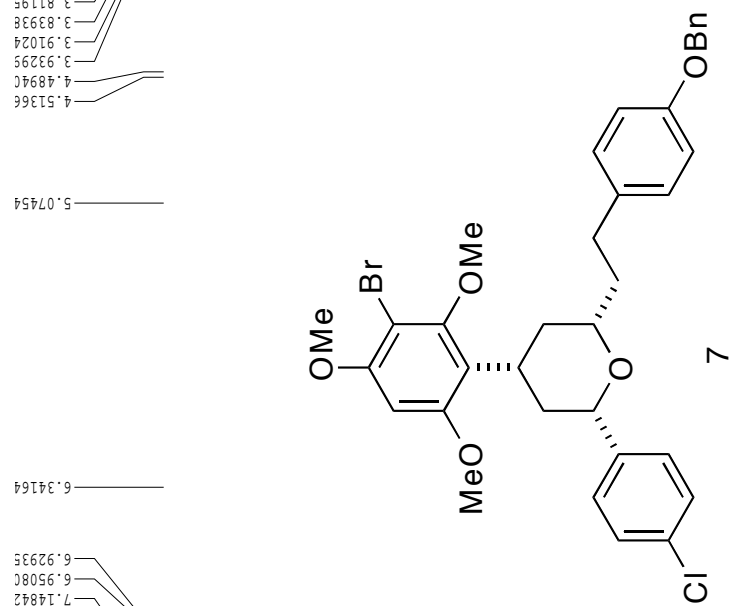

ระ626.9-

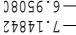

I8695: 2

$5869 \tau^{\circ} \mathrm{L}$

$96692 \mathrm{C} \longrightarrow$

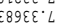

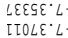

$5678 \varepsilon^{\circ}\llcorner-$

Bโ96r $2-$

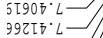

ग9वान:L-

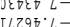

I6087. L 
Tian, Jaber, and Rychnovsky

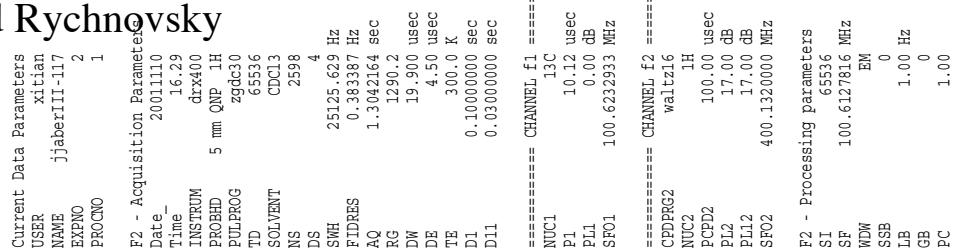

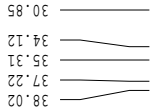

25.95
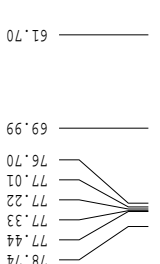

$8 \tau \cdot \varepsilon 6$

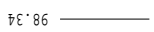

$79 \cdot \mathrm{FIT}$

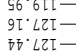

$7 \sigma^{\circ} \cdot \mathrm{LIT}$

$7 \mathrm{Z} \cdot 8 \mathrm{r \tau}$

$62 \cdot 82 \mathrm{~T}$

โร. $8 \mathrm{ZT}$

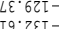

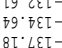

$08^{\circ} \operatorname{tzt}$

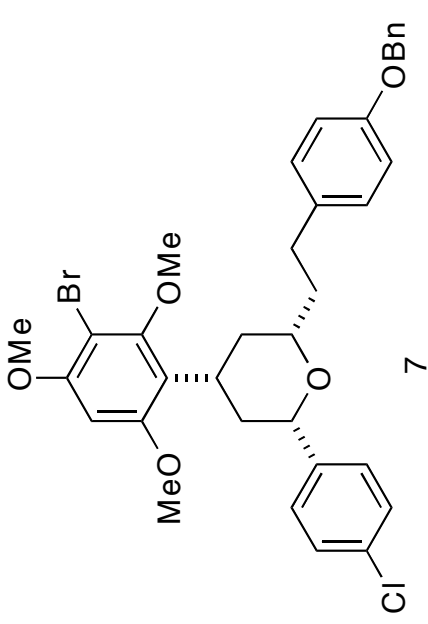

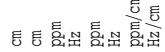
m

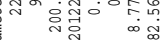

Page S-

19

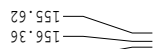


Tian, Jaber, and Rychnoysky
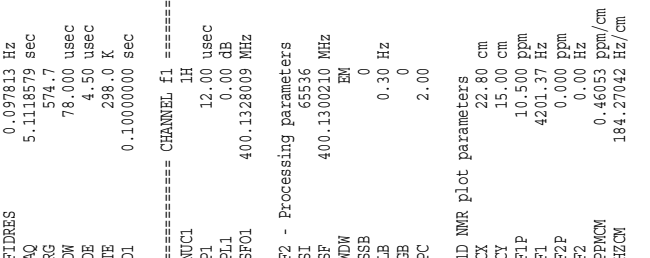

Page S-
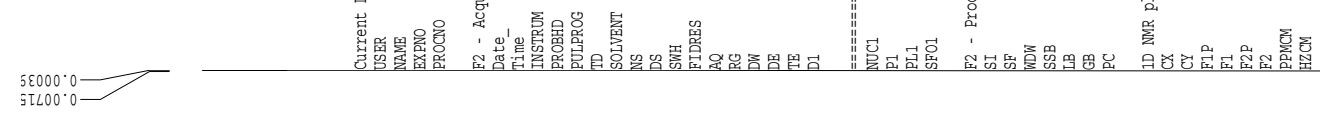

$\varepsilon \varsigma 6 \nsucc \varsigma^{\circ} \cdot$
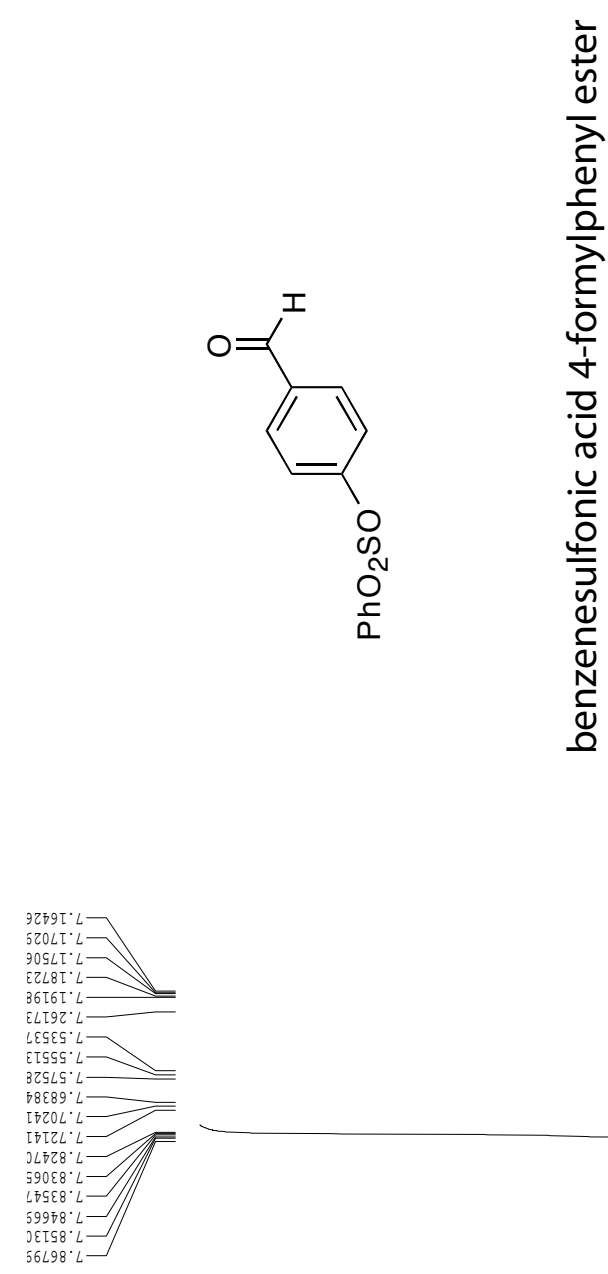

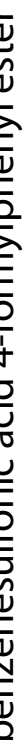

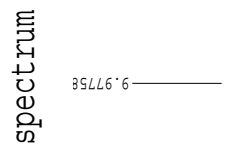

๒㯺 wdd 
Tian, Jaber, and Rychnovsky

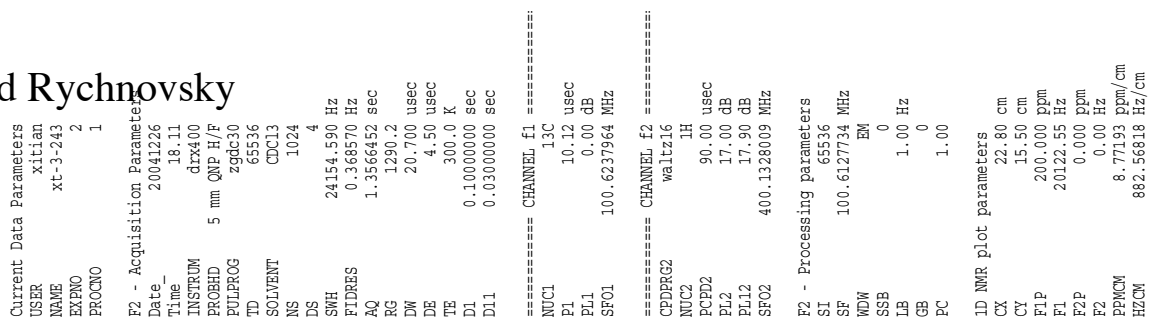

$69^{\circ} \cdot 9 L$

$00^{\circ} \mathrm{LL}$

$90^{\circ} \varepsilon Z \tau-$

$D^{\circ} \cdot 82 \mathrm{~T}$
$9 \varepsilon \cdot 62 \mathrm{~T}$

$8 \tau^{\circ}$ เ

เด. ซยโ-

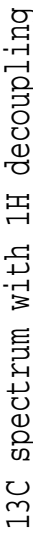

$\stackrel{\bar{d}}{\grave{d}}$

\}

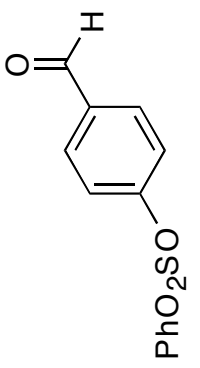

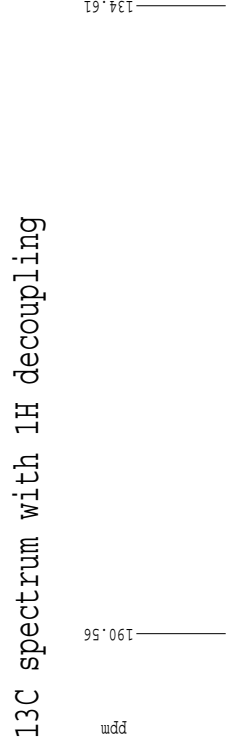

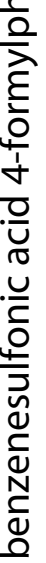

Page S-

21

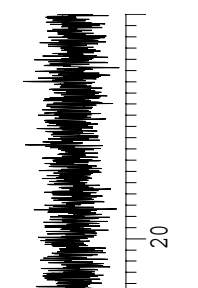


Tian, Jaber, and Rychnovsky

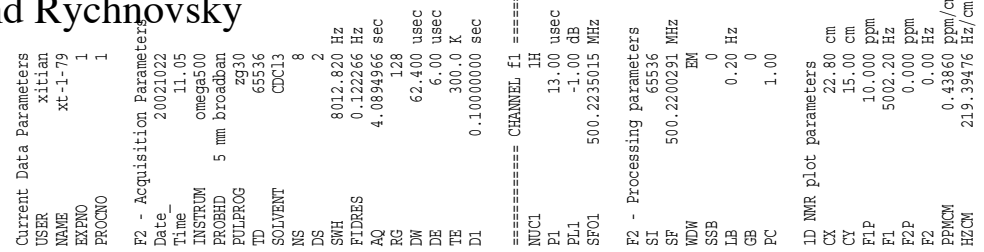

Page S-

22

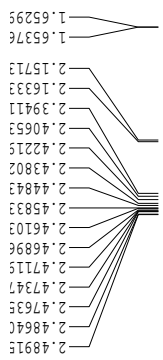

$\angle 6889^{\circ} 7$

$66269^{\circ} \mathrm{t}$
$3000 L^{\circ} \mathrm{t}-\mathrm{T}$

JELOL'T

ז09זL 7

$3660 \mathrm{~T} \cdot \mathrm{c}$

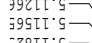

ร28It' 9

$39870^{\circ} \cdot 9$

970TL. 9

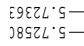

$306 \varepsilon L^{\circ} \mathrm{G}$

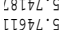

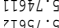

$6909 L^{\circ} \mathrm{s}$

$38796 \cdot 9-$

ESTLL' 9

БT8LL: 9

गह08L' $\mathrm{ST}$

$5786<\cdot \mathrm{s}$

Бெळ\&6.9-

$26686.9-$

$\angle \varepsilon 76.9$

ZLLS6.9-

39296.9

$30092: L$

$30092^{\circ} \mathrm{L}$

$\exists \mp \varepsilon L Z \cdot L$

$\overline{I S L L Z \cdot L}$

उहعО ' $L$

$2990 \mathrm{~S}^{\circ} \mathrm{L}$

I26 T' $L$

$\lfloor\angle 979 \cdot:$

76879.

5हा $99^{\circ} \mathrm{L}$

$56 \varepsilon 99^{\circ} \mathrm{L}-$

$389 \angle 9^{\circ} \mathrm{L}-$

ET $6 \angle 9 \cdot \mathrm{L}$

59089.2

$56 \varepsilon \tau 8 \cdot L$

$7918 \cdot 2$

15628.4

50 TE8. $L$

통

告
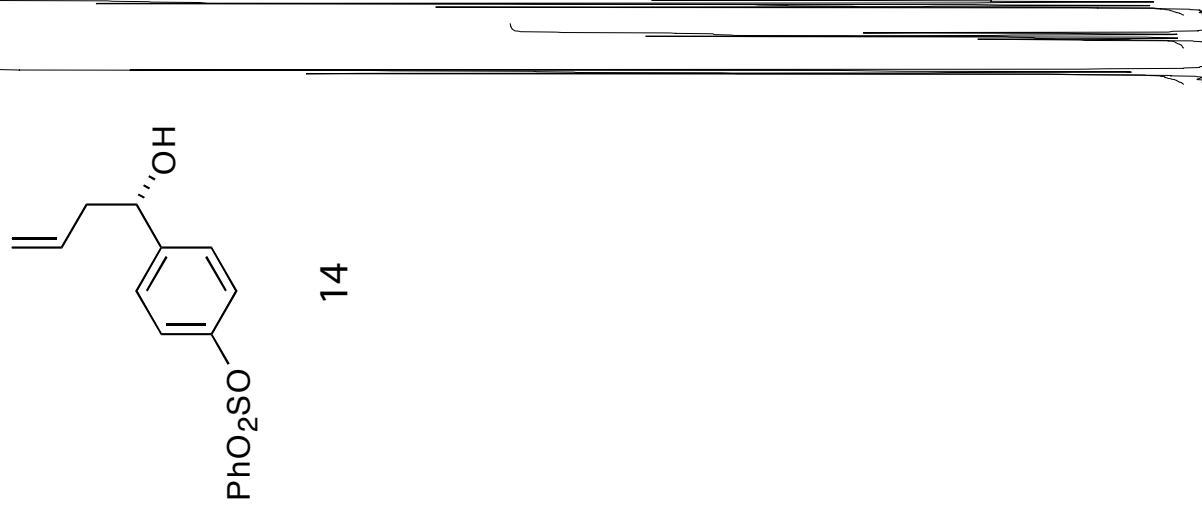
Tian, Jaber, and Rychnovsky

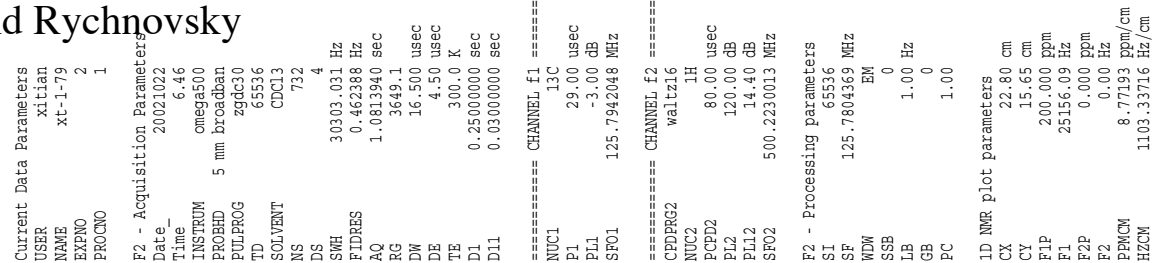

Page S-

$08^{\circ} \varepsilon \overline{1}$

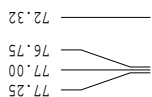

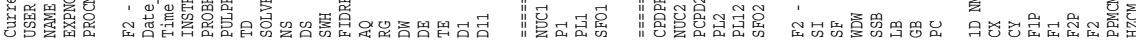

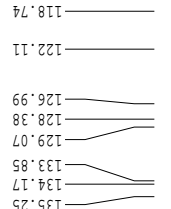

$88^{\circ} \mathrm{Z \pi t}$

$69 \cdot 87$

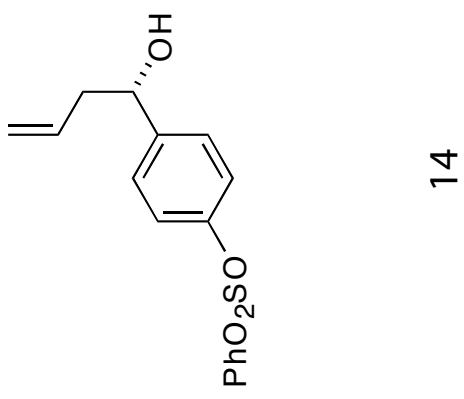


Tian, Jaber, and Rychnovsky

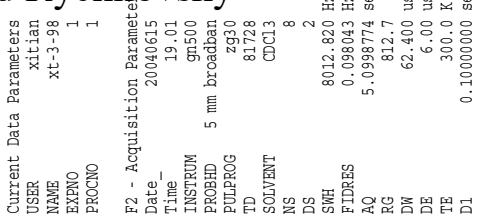

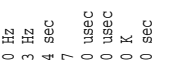

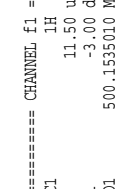

Page S-

24

$38590^{\circ} 2$

โ68ع $2 \cdot z$

LIfS9
$79699^{\circ} Z$

$36 \varepsilon \tau 6^{\circ} \mathrm{r}-$

$67626^{\circ} \mathrm{Z}$

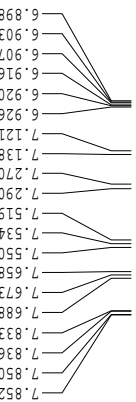

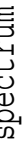

乲

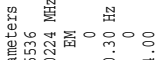

党, 学

竞

品

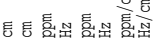

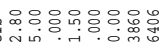

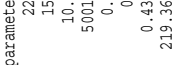

总

喑
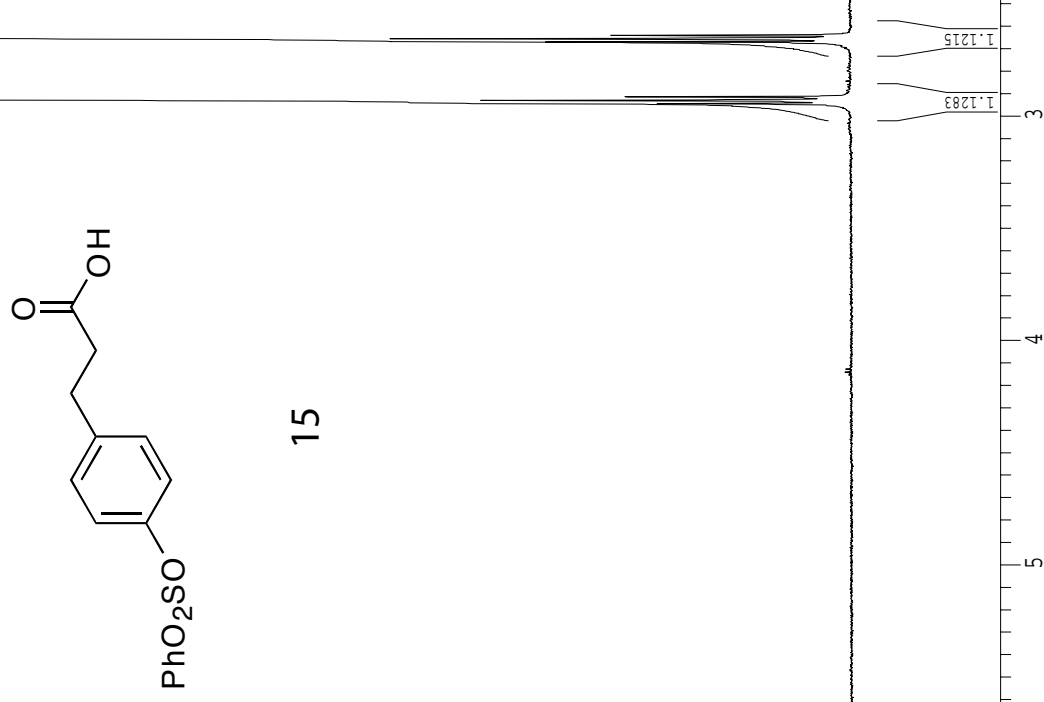

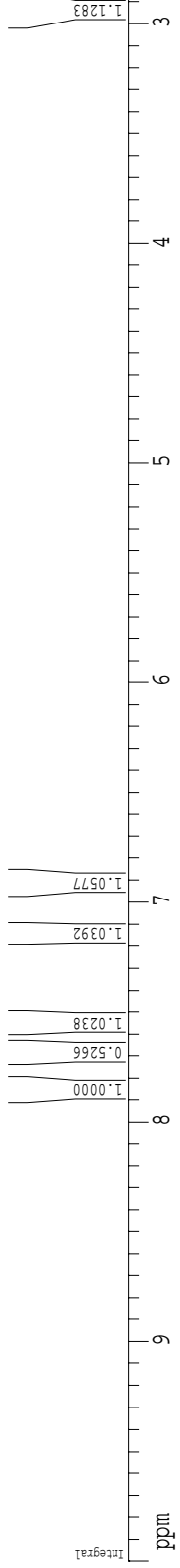


Tian, Jaber, and Rychnovsky

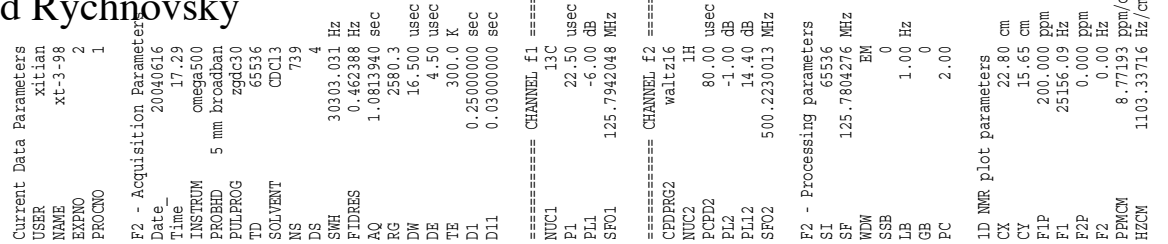

Page S-

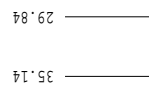

$S L \cdot 9 L$
$00^{\circ} L L$
$9 Z^{*} \angle L$

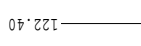

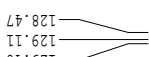

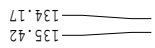

$\varpi \tau \cdot 6 \varepsilon \tau$

$70.87 \tau$

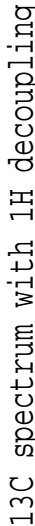
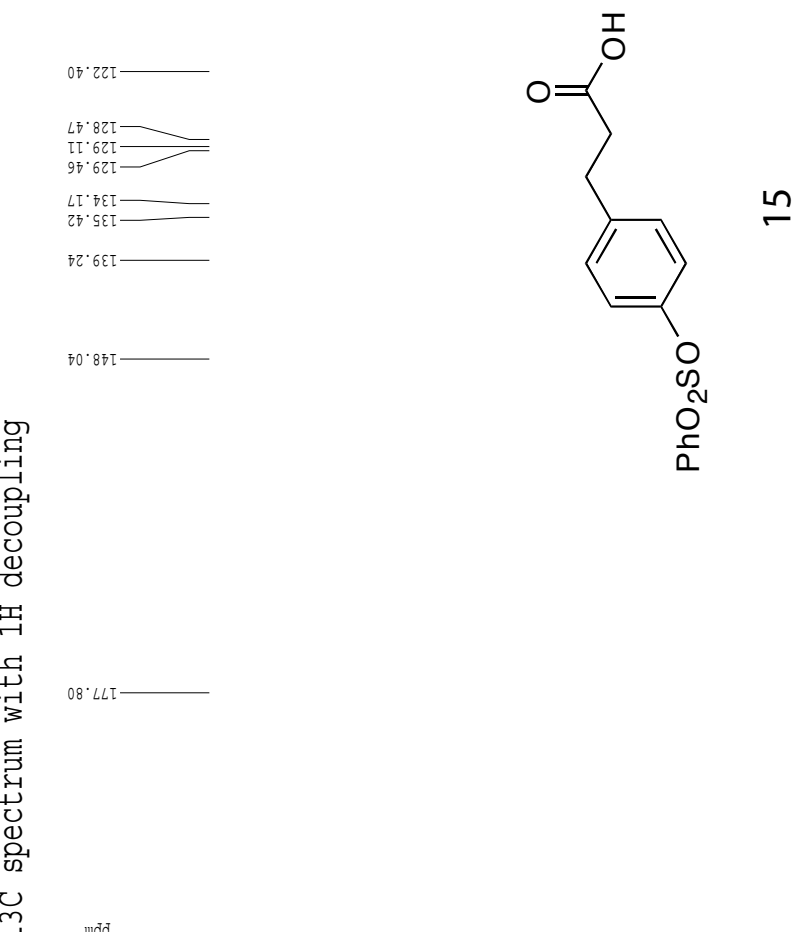
Tian, Jaber, and Rychnovsky

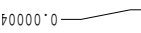

$56788^{\circ} \cdot 0$

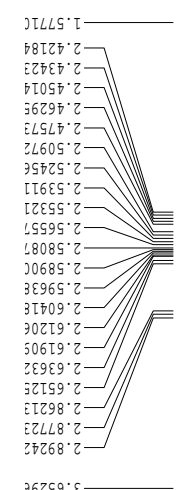

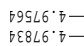

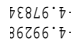

$36266^{\circ} 7 \longrightarrow$

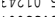

$10085.9-$

JSTSg. 9

$3 \mathrm{~T} 89 \mathrm{~S}^{\circ} \mathrm{S}$

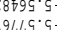

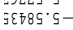

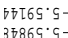

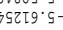

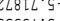

эตรEL' 9

$\angle 8976.9$

$3288 L \cdot 9$

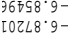

20786.97
6825.9

$68 \mathrm{~T} 96.9$

$30 \mathrm{~T} 50^{\circ} \mathrm{L}$

$36 \angle 90^{\circ} \mathrm{L}$

IL99T' $L$

$56 \varepsilon 8 \mathrm{~T}^{\circ} \mathrm{L}$

iteOS:L

J0ETS $L$

$0675^{\circ}$

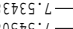

$8979 \cdot 6$

¡ह899. $\mathrm{L}$

Coع L9: L -

$6 \angle 89^{\circ} \mathrm{L}-$

โ88โ8. L

.

$6928^{\circ} \mathrm{L}$

ᄂL988. L

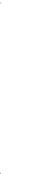

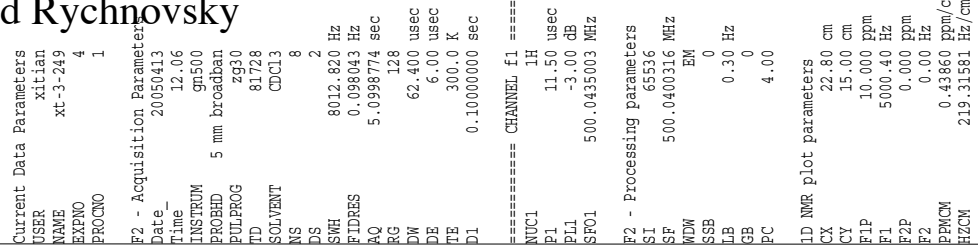

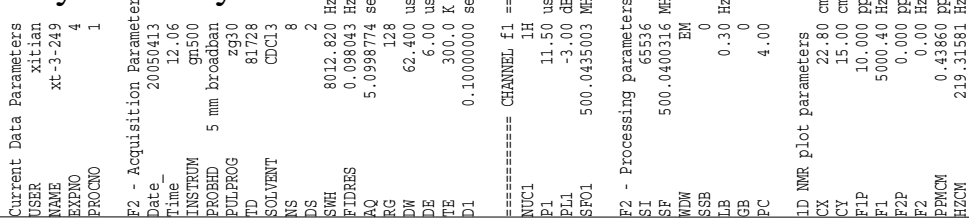

Page S-

26
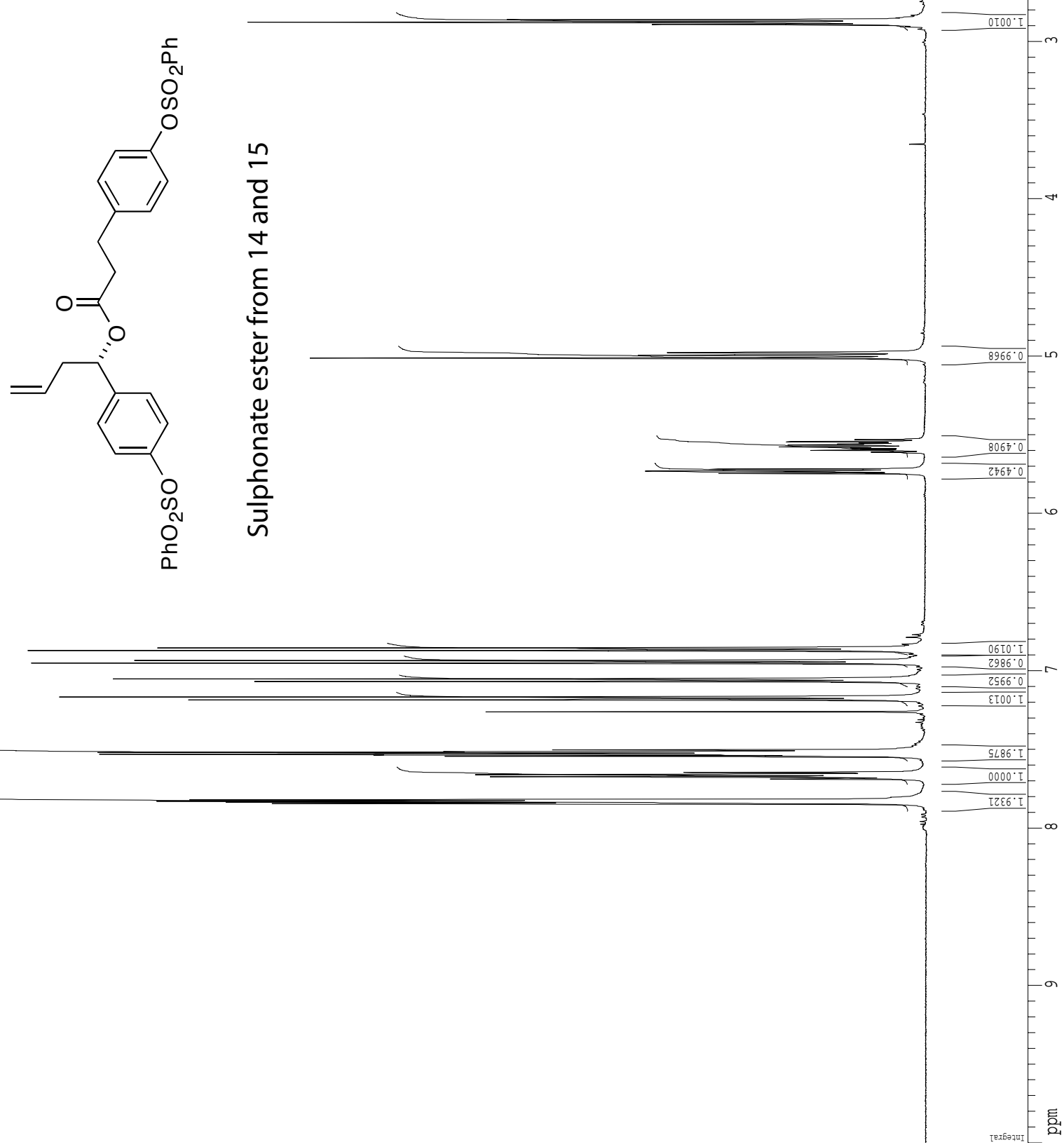
Tian, Jaber, and Rychnovsky

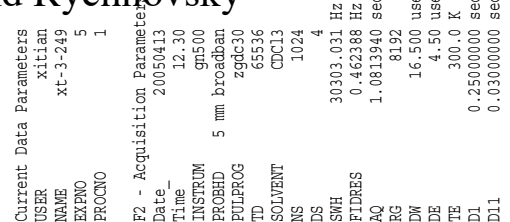

00.0

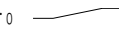

Page S-

27

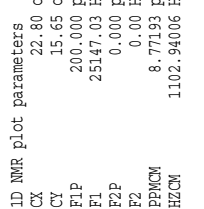

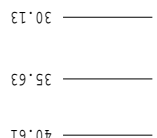

$\varepsilon E^{\circ} \mp L$
$8 L \cdot 9 L=\square$
$\varepsilon 0^{\circ} \angle L$
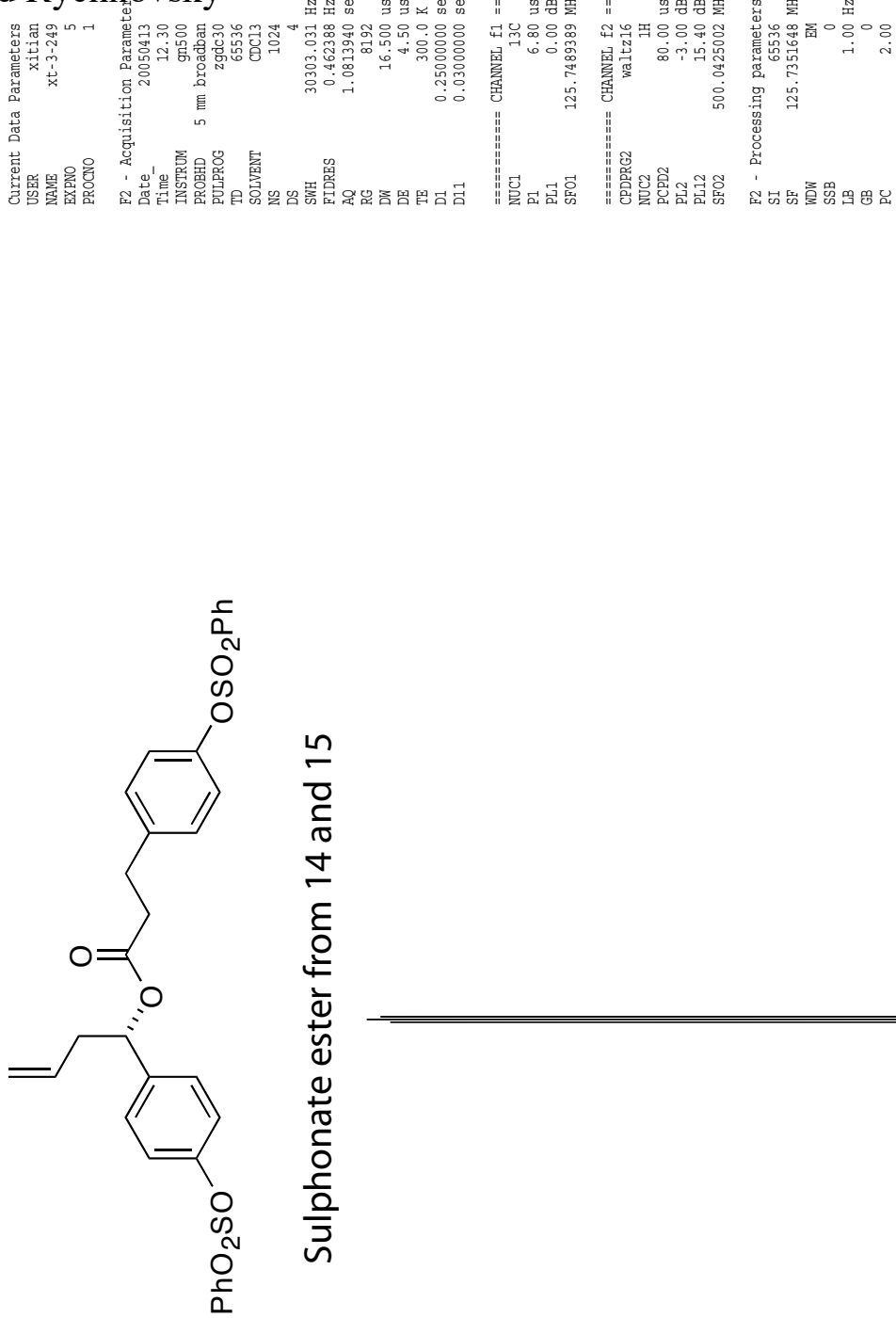

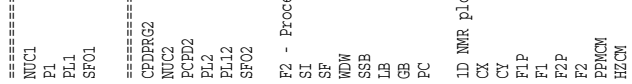
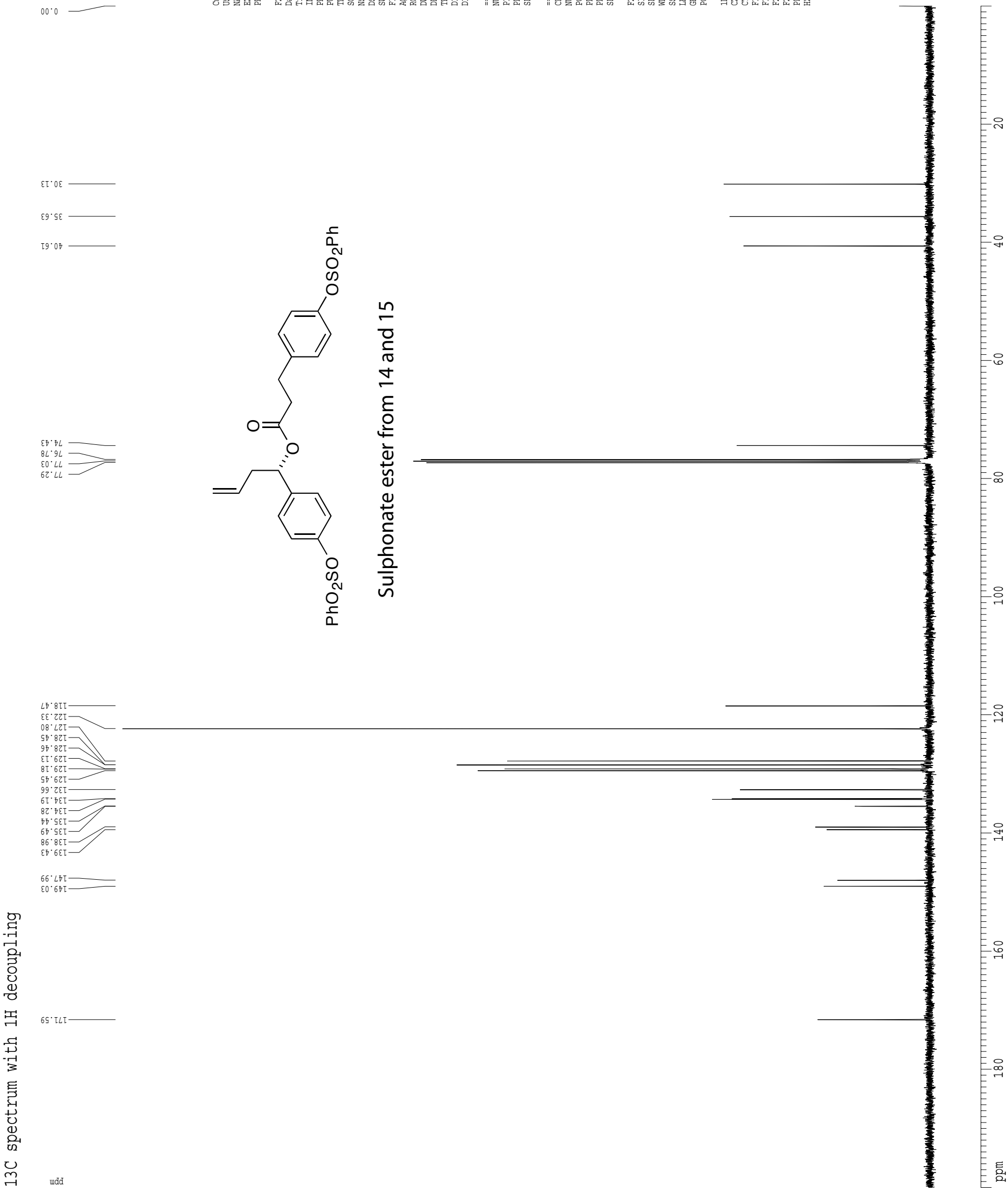
Tian, Jaber, and Rychnovsky

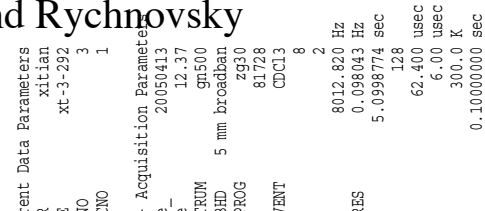

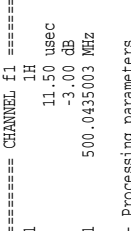

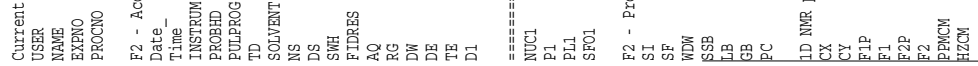

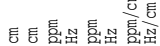
品

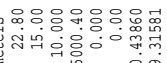

Page S-

28

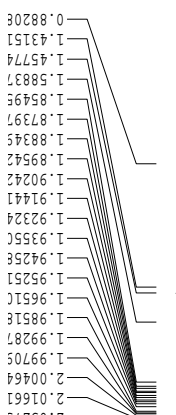

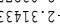

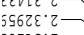

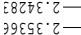

iTIST:Z

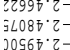

$62609^{\circ} 2$

$626 \mathrm{Tg} \cdot 2$

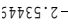

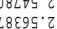

$\bar{z} 29 \varepsilon 9 \cdot 5-5$

$\varepsilon 6879 \cdot 2-$

j7599. 2 -

$3 \angle 899^{\circ} 2=$

$38889 \cdot 2$

$29004 \cdot 7$

$36087^{\circ}+\bar{T}$

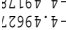

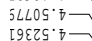

ธะ9\&S $7-$

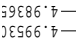

$28666^{\circ} 7-$

อร9โ0.5

$7980 \cdot 9$

$57989^{\circ} \mathrm{s}$

TS909.5 는

59989.9-

$50279 \cdot 5$

$39679^{\circ} 9$

ELZ99. 9

$\rightarrow \varepsilon 9 L 9 \cdot c$

ह8 $8289 \cdot 9$

$39069 \cdot 9$
$5 \angle 969 \cdot 9$

$62969 \cdot 9$

$30646^{\circ} \mathrm{C}$

20626.

$57628 \cdot 9$

$3 \varepsilon 978.9-$

$528 \angle 8 \cdot 9$

โ2968.9-

$6\llcorner\varepsilon 6.9-$

วSโ76.9-

โ6756.9-

IIZLO ${ }^{\circ} \mathrm{L}$

$30680^{\circ} \mathrm{L}$

$679 \mathrm{LI} \cdot \mathrm{L}$
$5788 \mathrm{~T}^{\circ} \mathrm{L}$

$50 \varepsilon 6 T^{\circ} L$

IOL8D: $L$

ZLLOS. $\angle$ L

ELETS : $L$

39عZ5: $\circ$

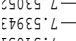

I $997 \mathrm{~F}^{\circ} \mathrm{L}$

$9679 . \mathrm{L}$

$7959^{\circ} \mathrm{L}-$

$9 \varepsilon 99^{\circ} \mathrm{L}-$

$57889^{\circ} \mathrm{L}$

$\varepsilon 908^{\circ} \mathrm{L}$

$8128^{\circ} \mathrm{L}$

志

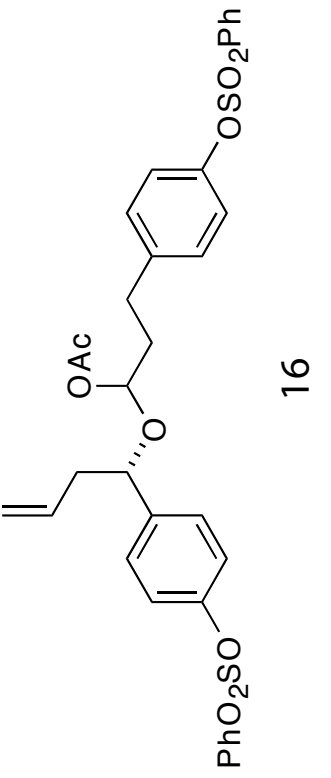

\section{促}


Tian, Jaber, and Rychnovsky

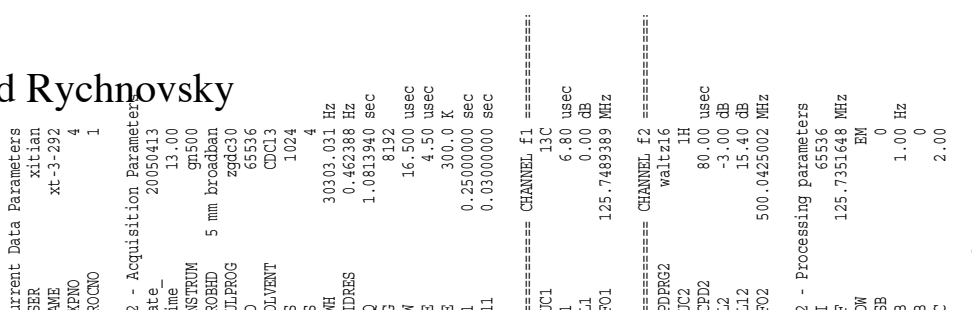

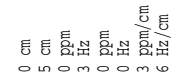

Page S-
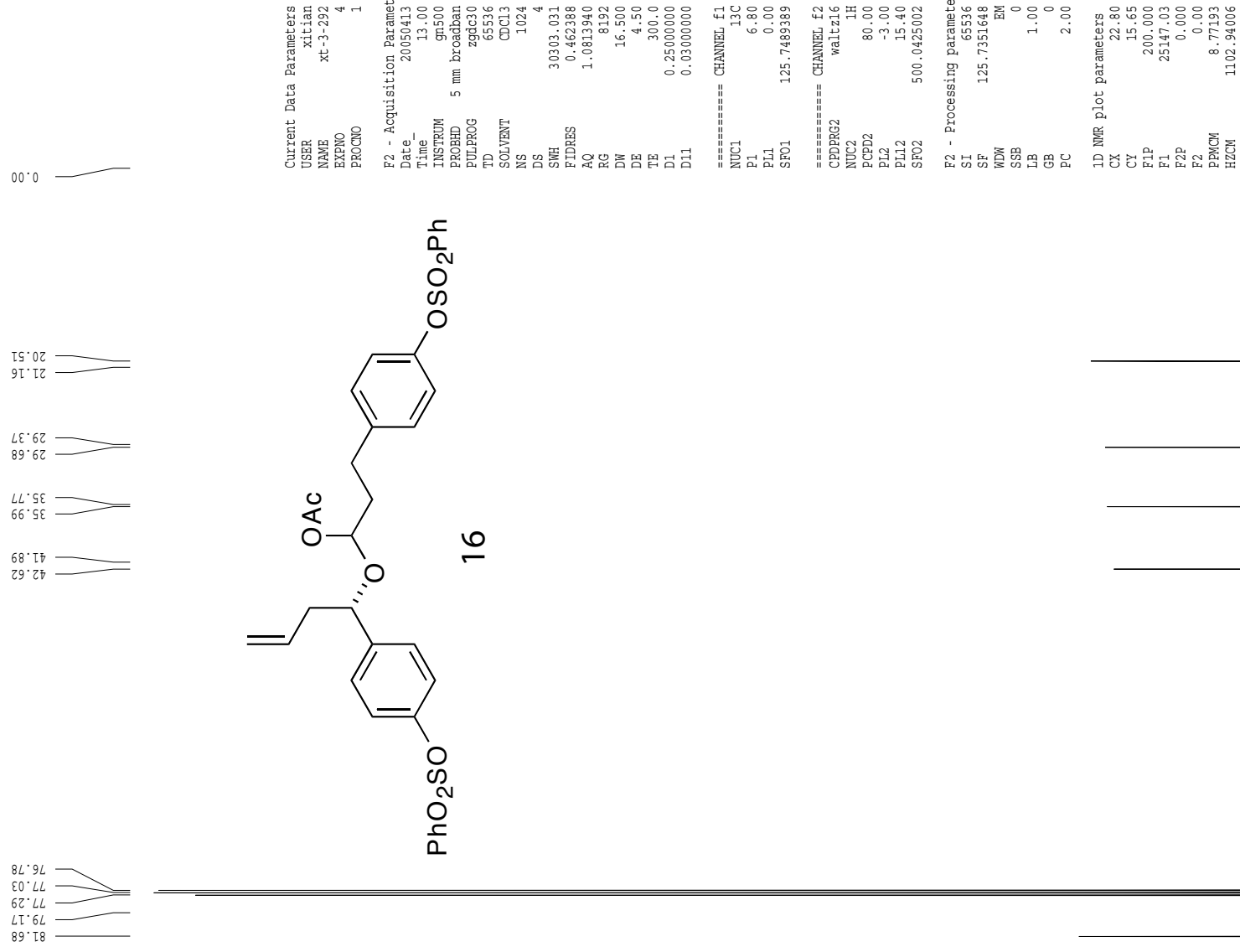

$6 \mp \cdot 96$
$S F \cdot L 6$

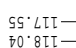

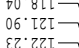

$82 \cdot 28 \tau]$

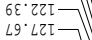

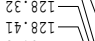

Sन.8ZT]

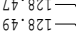

2T. 627

LI $62 \mathrm{CI}=$

28: $627=$

ID.62T-

TL. हEI $\longrightarrow$

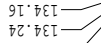

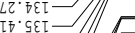

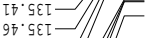

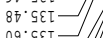

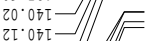

Or. ODI

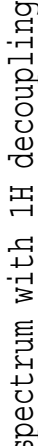

की

I8. LIT

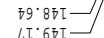

잉

${ }_{8 L^{\circ} \circ L L T=}$

छ

, 
Tian, Jaber, and Rychnovsky
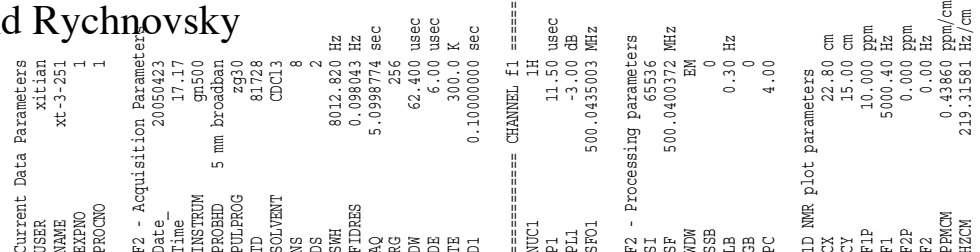

Page S-

30

[9เZ9

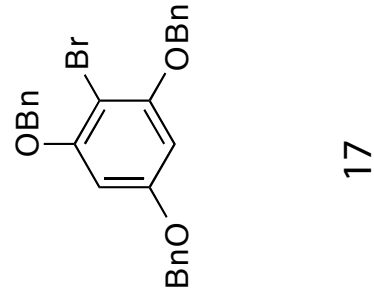

$19 \angle 96^{\circ} 7$

$\mp \mp L 8 Z \cdot 9$

"|

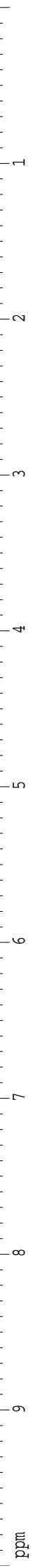




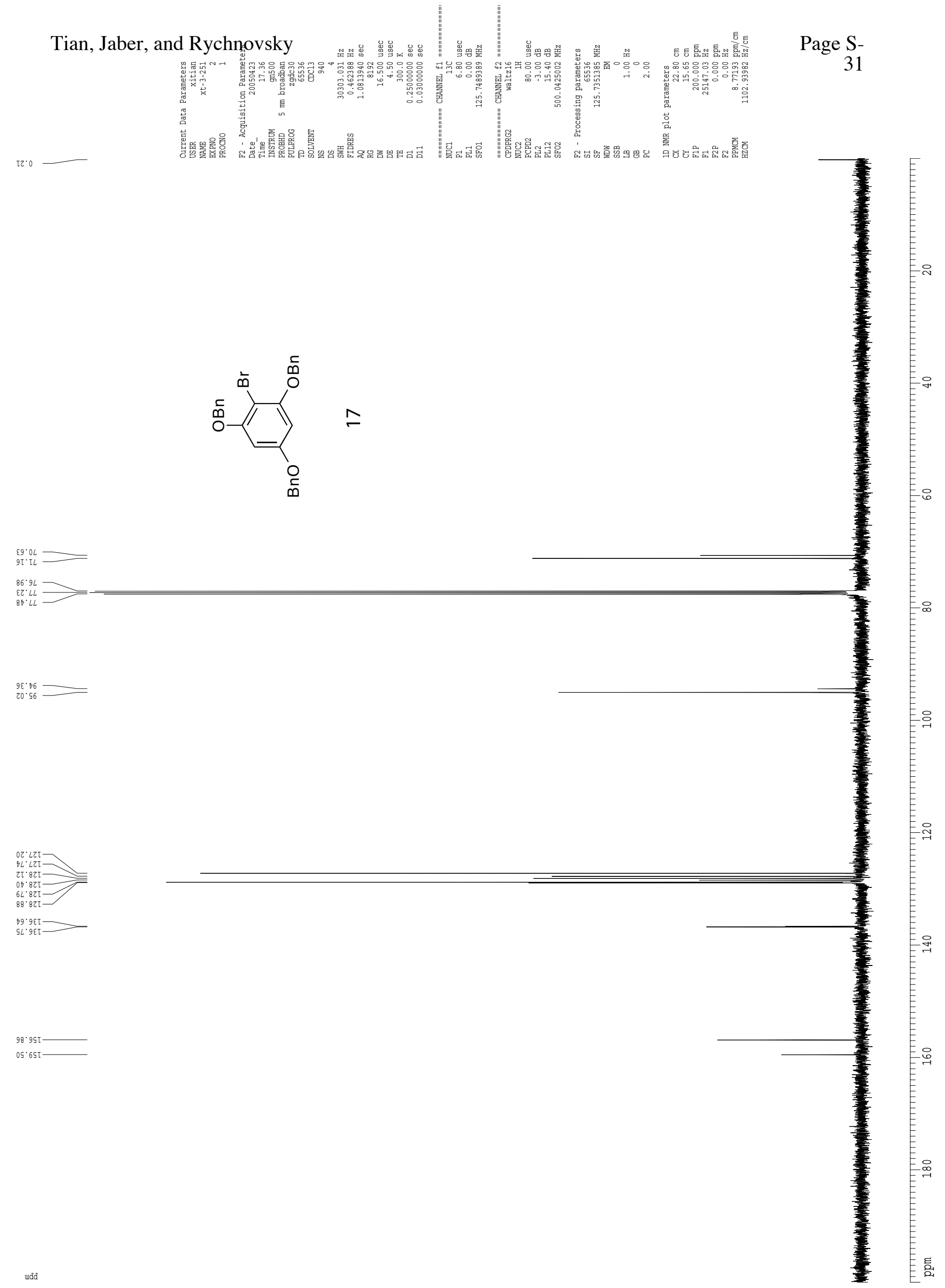


Tian, Jaber, and Rychnovsky

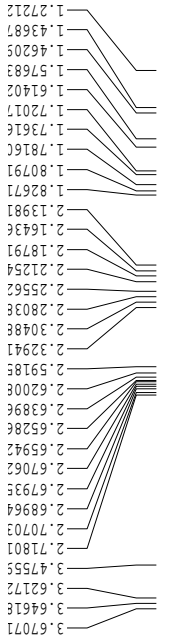

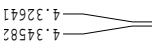
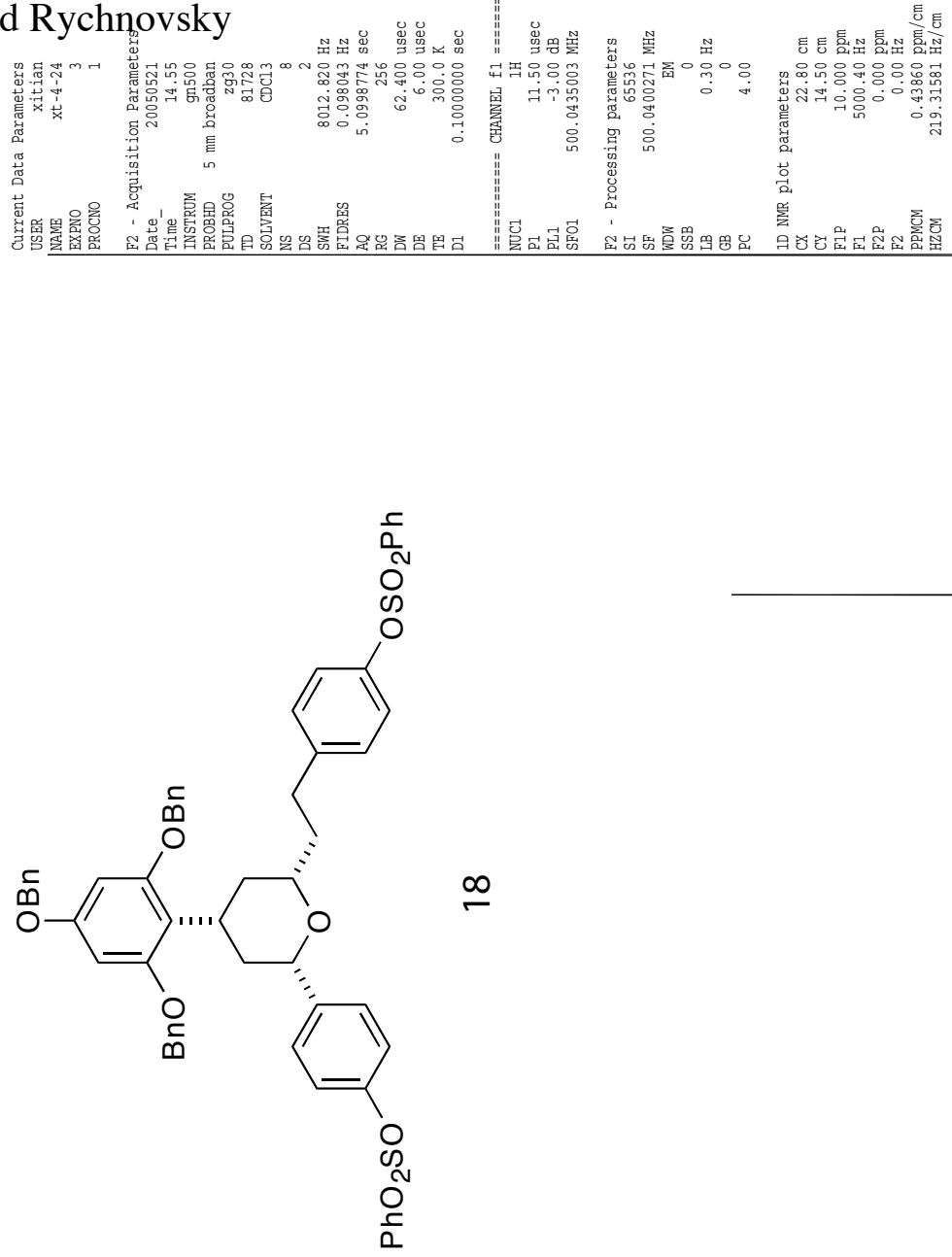

$\infty$

$36 \varepsilon 66^{\circ}$ Ð

500 T0. $\mathrm{s}$

$38980^{\circ} \mathrm{g}$
$\mathrm{L} T \mathrm{~T} 90^{\circ} \mathrm{S}$

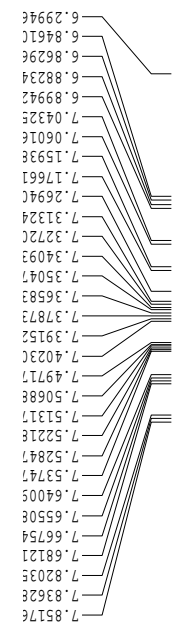

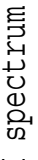

㞼 wad
Page S-

32 
Tian, Jaber, and Rychnovsky

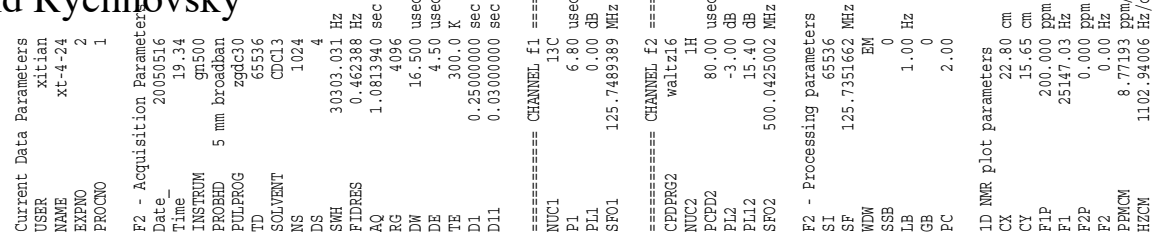

$98 \cdot 0 \varepsilon$
$\bar{T} \cdot 2 \varepsilon$

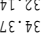

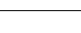

${ }_{\varepsilon q} \cdot\llcorner\varepsilon=$

$8 \mathrm{~L}^{\circ} \mathrm{OL}$
$08.0 \mathrm{C}$

$08^{\circ} 02$

$L \cdot 9 L$
$20 . L L$

$\varepsilon Z \cdot L L \longrightarrow$

$\angle 2 \cdot L L=$

$\angle 0^{\circ} 76$

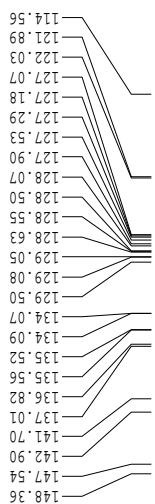

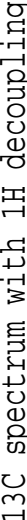

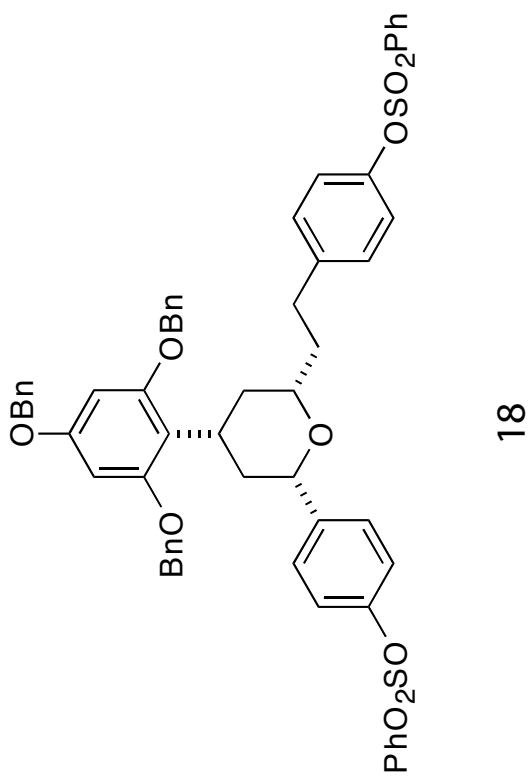

Page S-

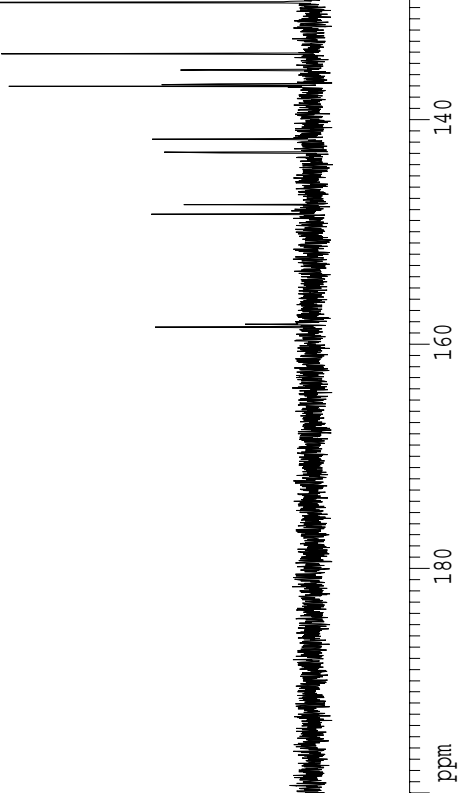


Tian, Jaber, and Rychnovsky

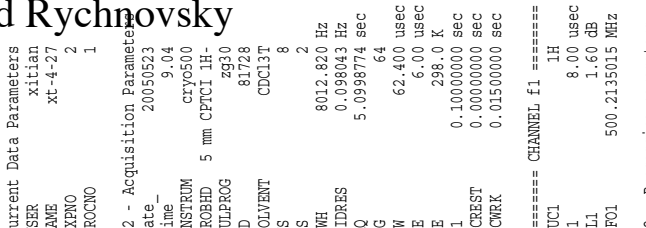

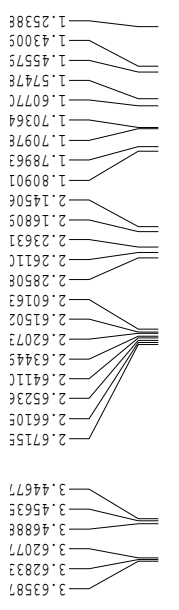

$\angle$ I $60 \varepsilon^{\circ} \sqcap \longrightarrow$

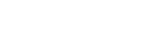

$3895 L^{\circ}$ ๆ

$39686^{\circ} \mathrm{t}$

$19070^{\circ} \mathrm{s}$

ᄂ962I:9

$6 \angle 28 \cdot 9$

с0578.97

ว६ร98.9

$99288^{\circ} 97$

$362200^{\circ} \mathrm{L}$

$\mathrm{JTOF0} \cdot \mathrm{L}$

โ927โ: 27

IZLSZ $\angle-$

$66808: L$
$3 \angle 6 \tau \varepsilon: L]$

उ8zzद. $L$

ZLGEE: $L-$

$2697 \varepsilon^{\circ} L-$

उहOSE $L$

$-899 \varepsilon^{\circ} L$

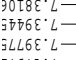

$\angle L 6 L F^{\circ} L$

SL L $\sigma^{\circ} L$

EItS:L-

GSSTS: $L$ -

उटहE9. $L$

$389 \varepsilon 9^{\circ} \mathrm{L}-$
$\mathrm{I} \sigma L \varepsilon 9^{\circ} \mathrm{L}$

$2879 \cdot:-$

EET08:L二

$56808^{\circ} L$

TERT8.L

ง $8858^{\circ} L$

$\exists L F \& B^{\circ} L$

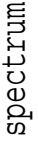

Page S-

34

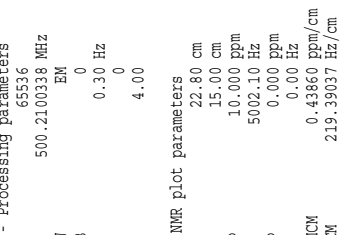

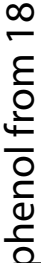

ำ
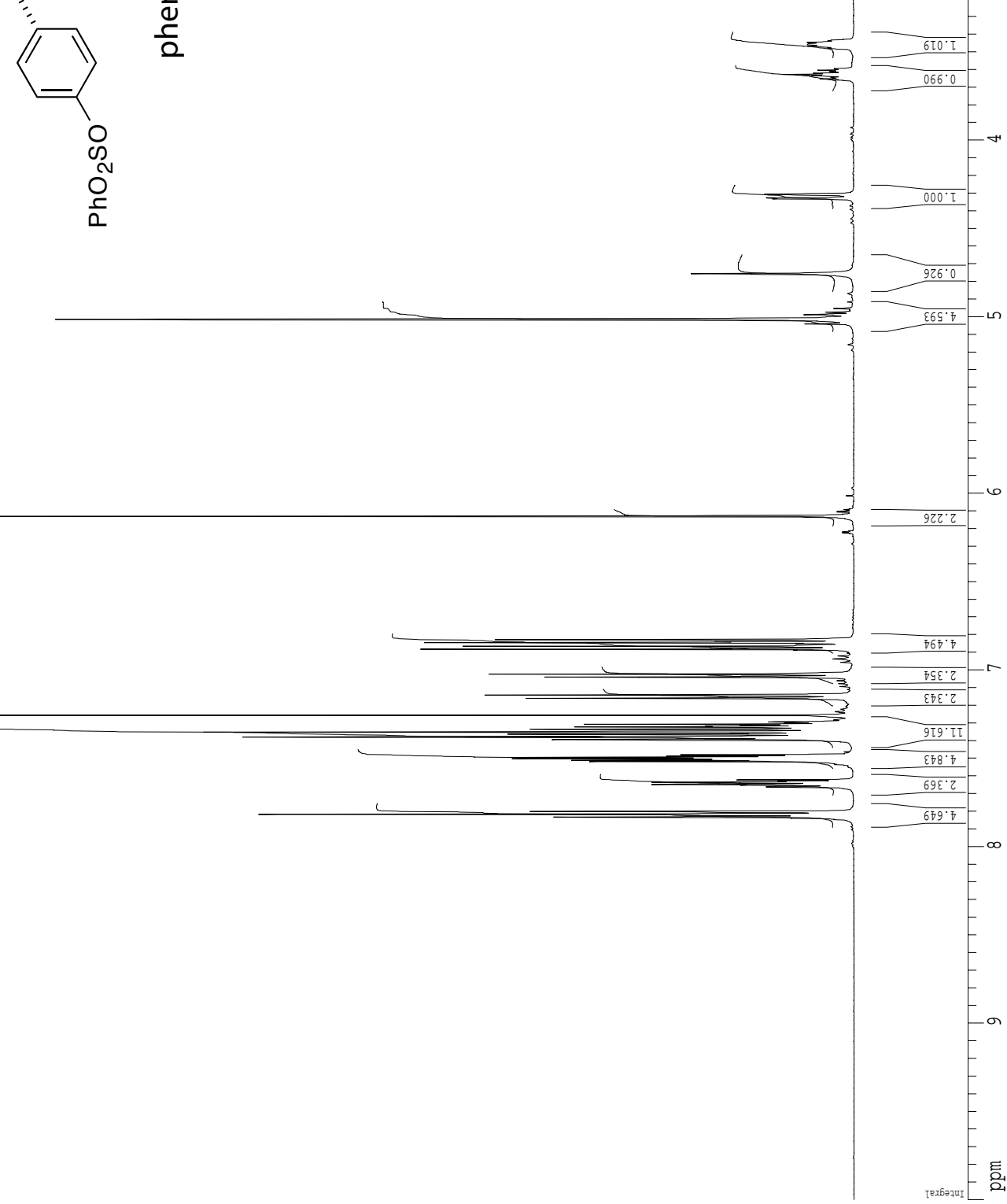
Tian, Jaber, and Rychnovsky

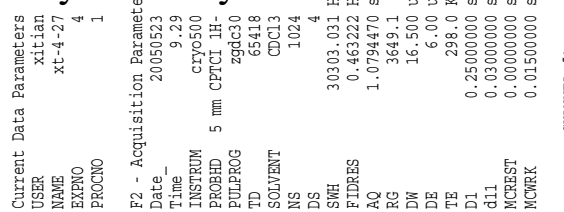

$98^{\circ} 0 \varepsilon$
$90^{\circ} \tau \varepsilon$
$9 \varepsilon^{\circ} \downarrow \varepsilon$
$8 \tau^{\circ} L \varepsilon$
$\tau 9^{\circ} L \varepsilon$

$59 \cdot 0 L$

$9 L \cdot 9 L$

$Z 0^{\circ} \angle L$
$L Z \cdot L L$

TT. $6 L$

$\varsigma Z^{*} \neq 6$
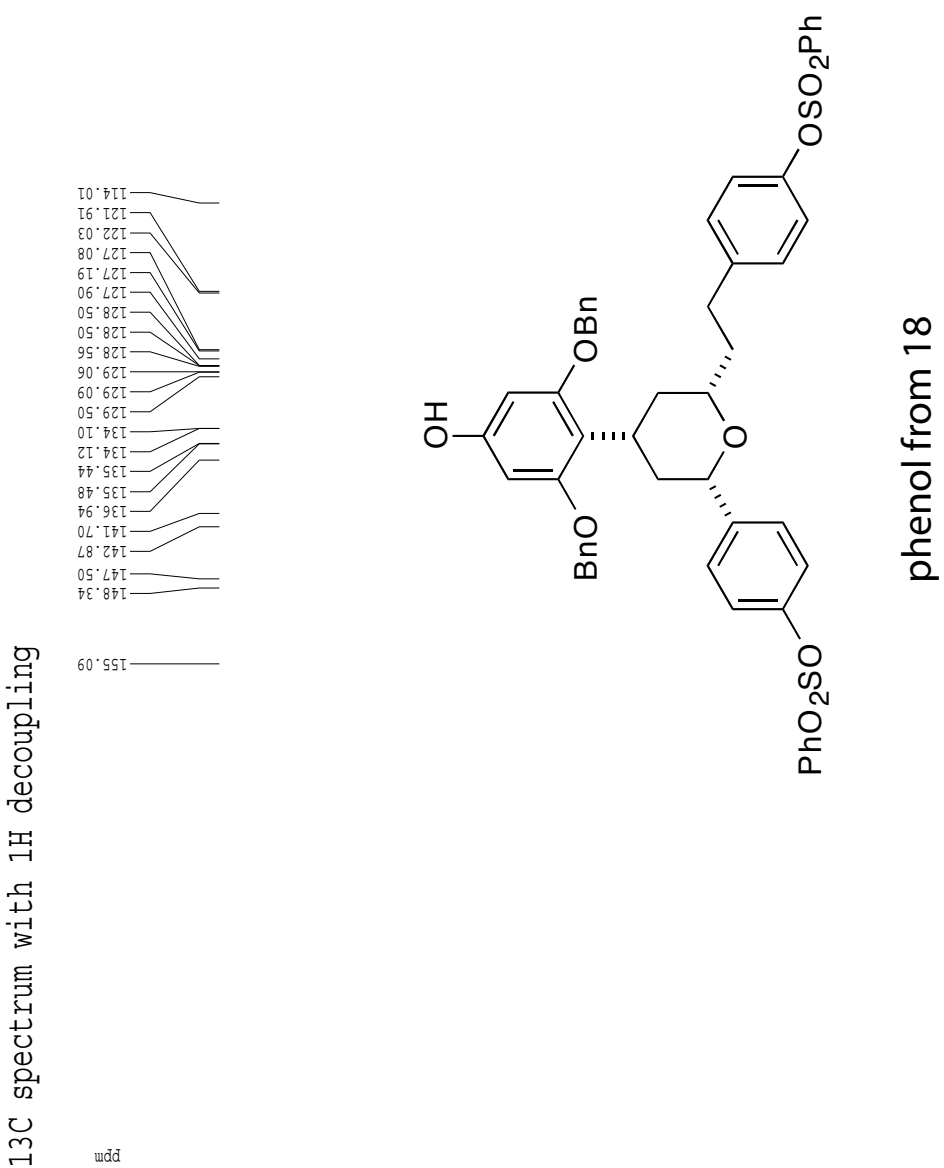

$\frac{\infty}{\frac{1}{0}}$
Page S- 
Tian, Jaber, and Rychnovsky

$30000 \cdot 0$

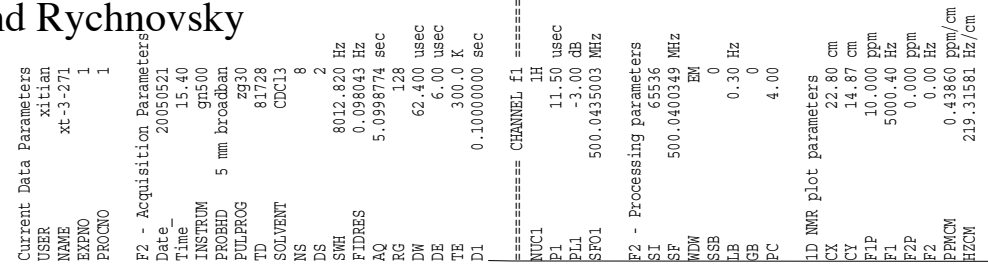

Page S-

36

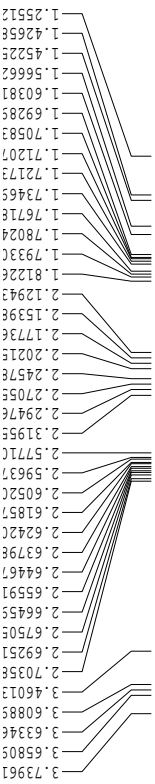

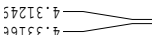

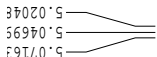

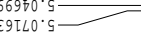

EโZ6Z' 9

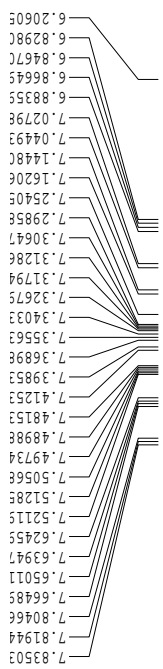

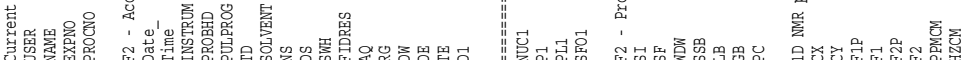

\section{(n)}

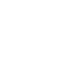
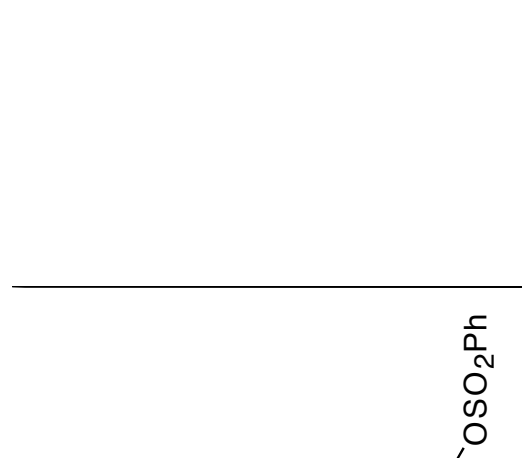

○
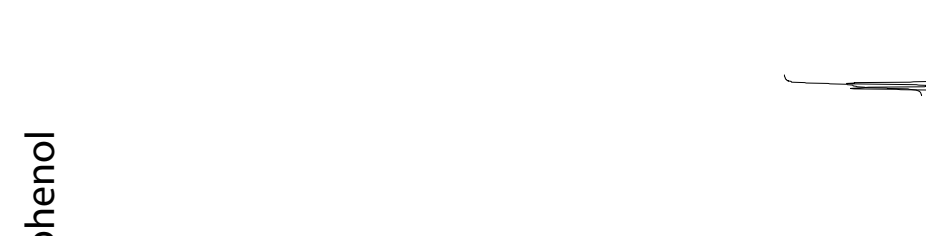

\section{(1)}


Tian, Jaber, and Rychnovsky

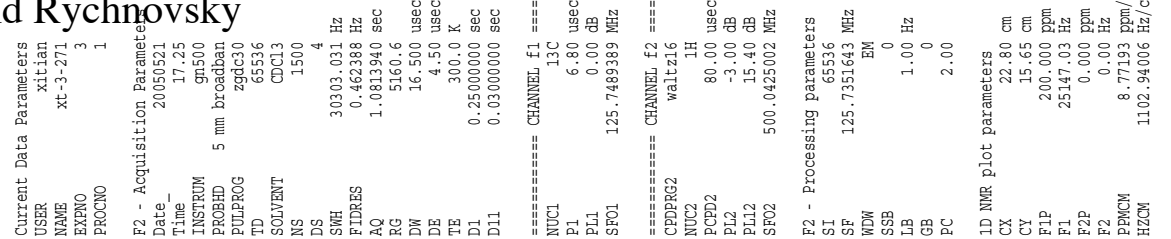

Page S-

37

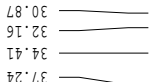

$7 g^{\circ} L \varepsilon=$

०६५५

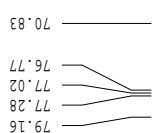

${ }^{\top} \Gamma^{\circ} \varepsilon \sigma$

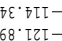

68. 72 7

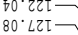

โร: $\angle 2 \mathrm{TI}$

โร. $82 \mathrm{ZT}$

$99 \cdot 82 \mathrm{~L}$

$90^{\circ} 62 \mathrm{I}-$

$60^{\circ} 62 \mathrm{~T}$

$\angle 0^{\circ} \mathrm{DE \tau}-$

6. $90^{\circ} \mathrm{\varsigma \varepsilon \tau}-$

$09 \cdot$ `ॄ _

ZL. IIt

ZL'䤊—

$99^{\circ} \angle A T$
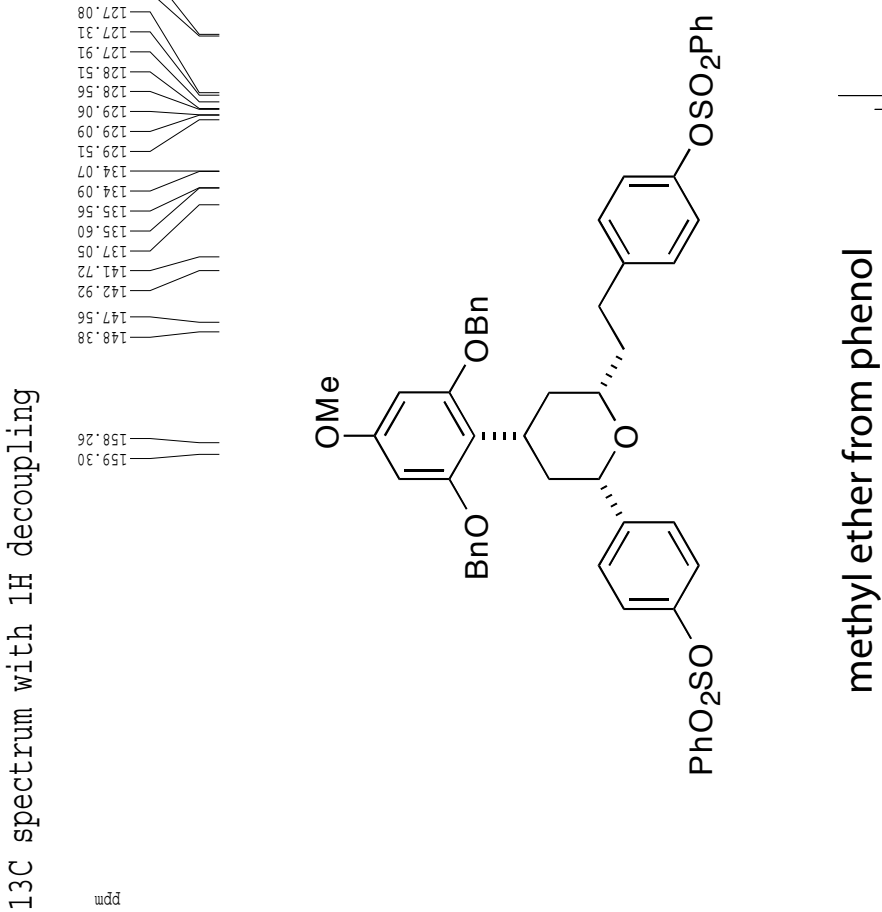

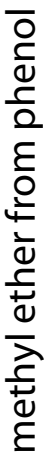

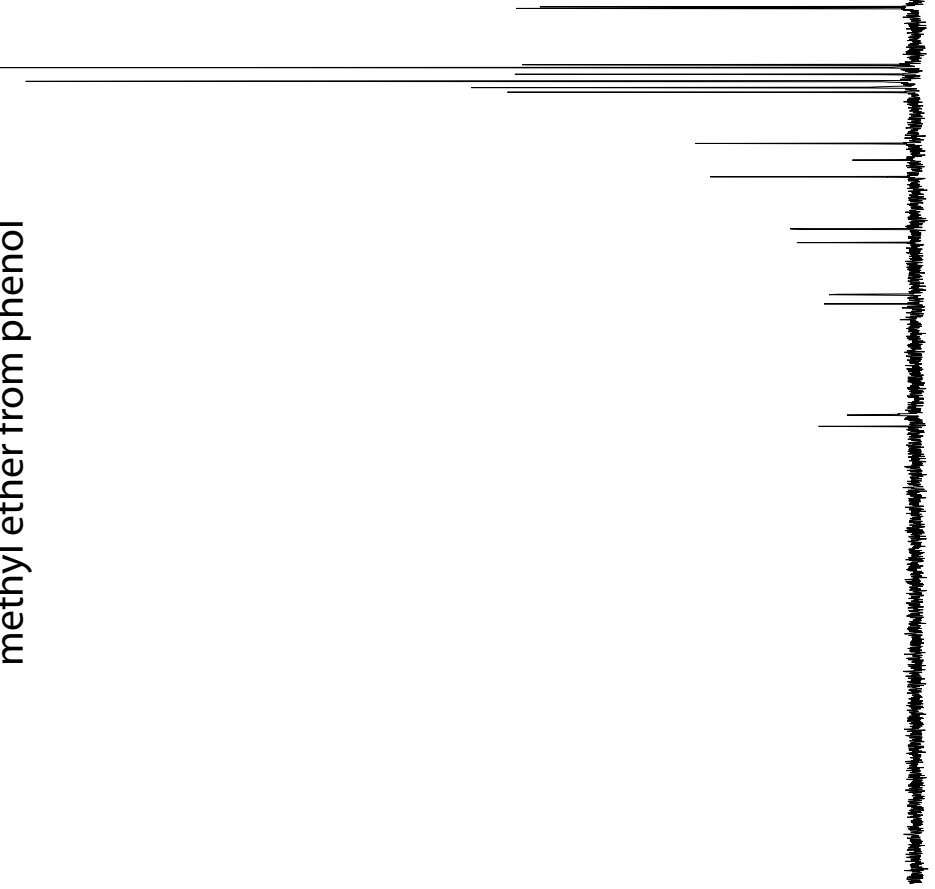


Tian, Jaber, and Rychnovsky
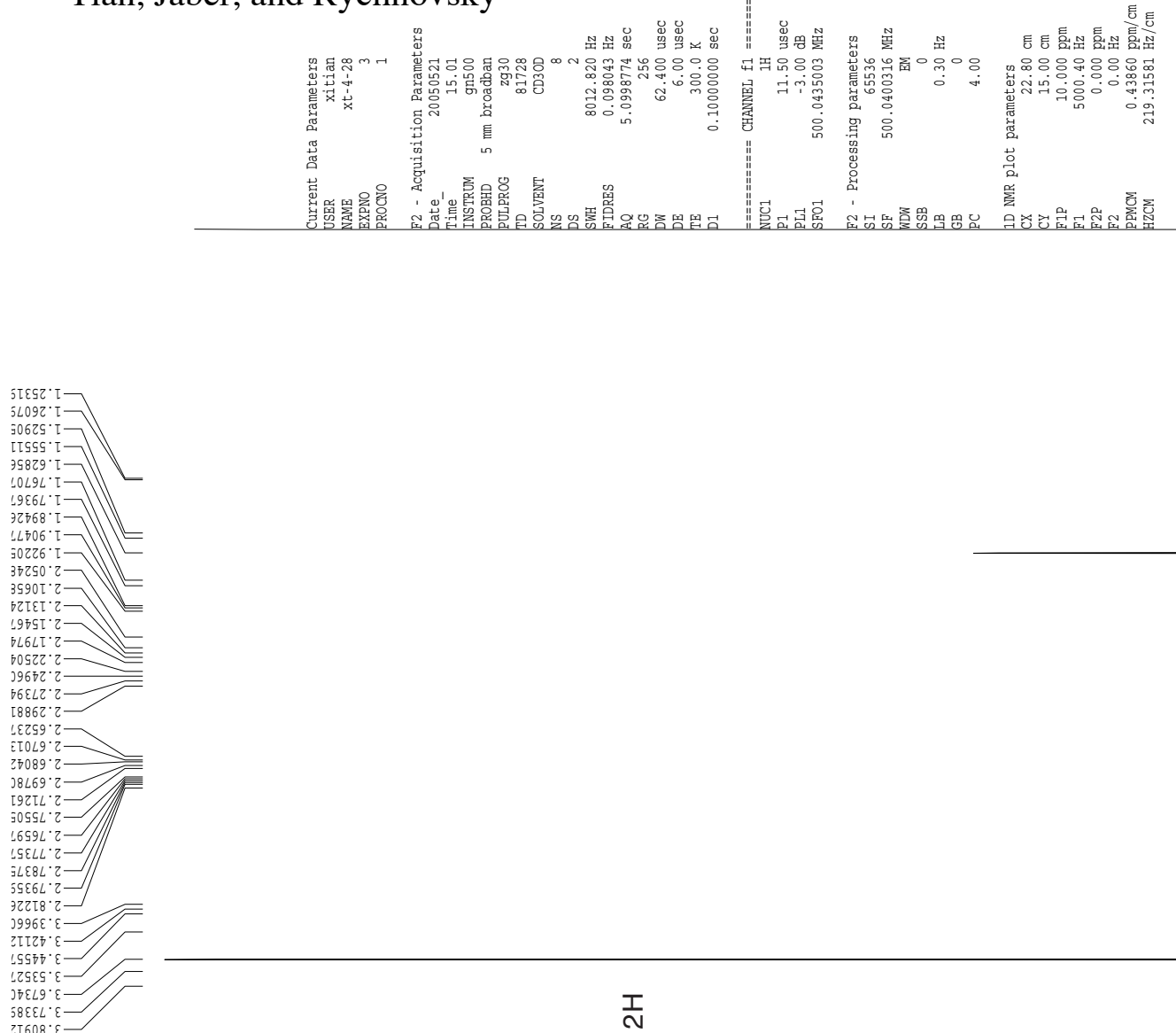

(T600.

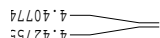

¿ZEฐ0.ร

톡
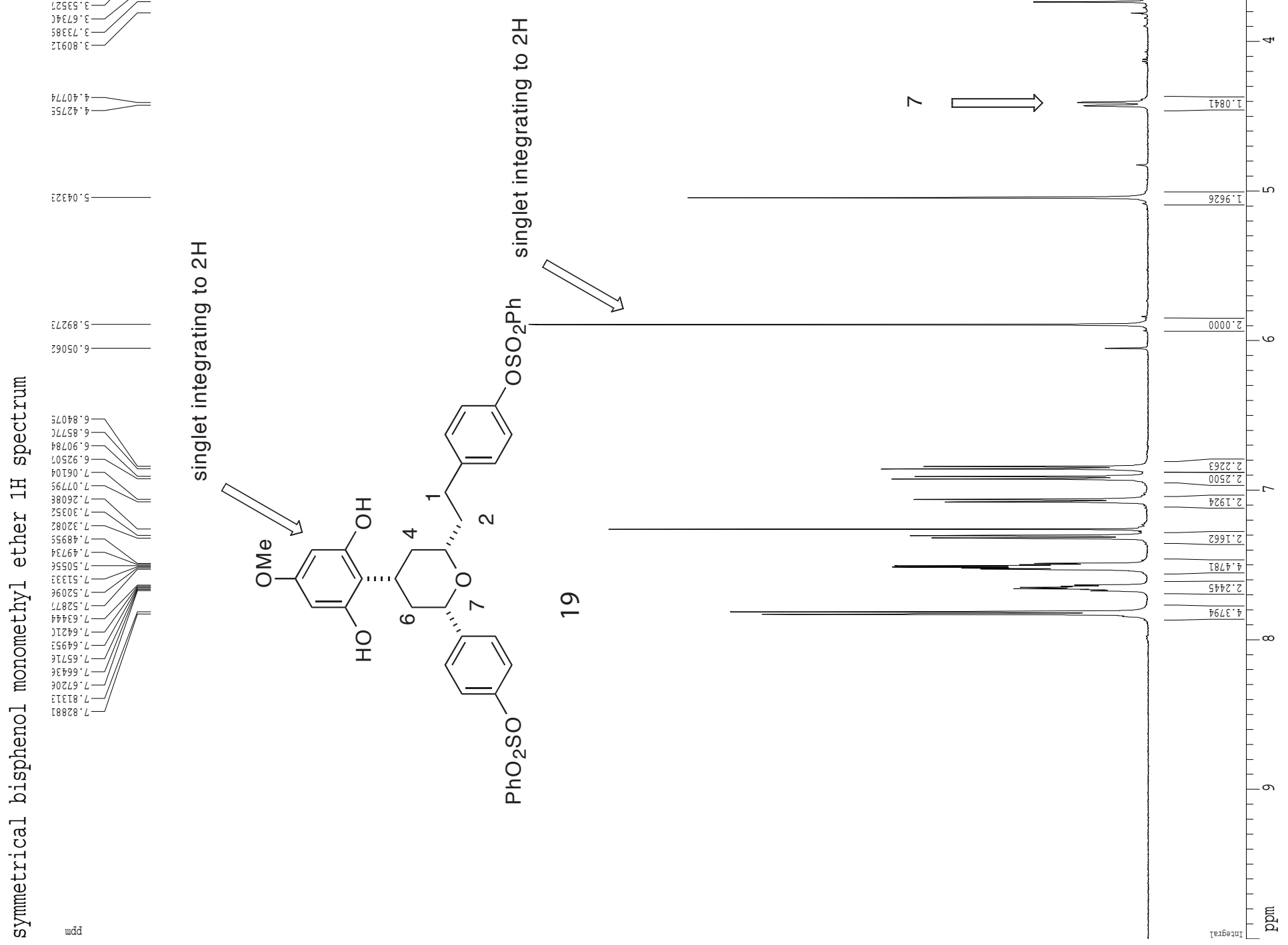
Tian, Jaber, and Rychnovsky

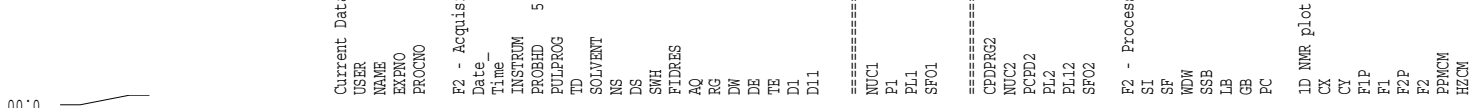

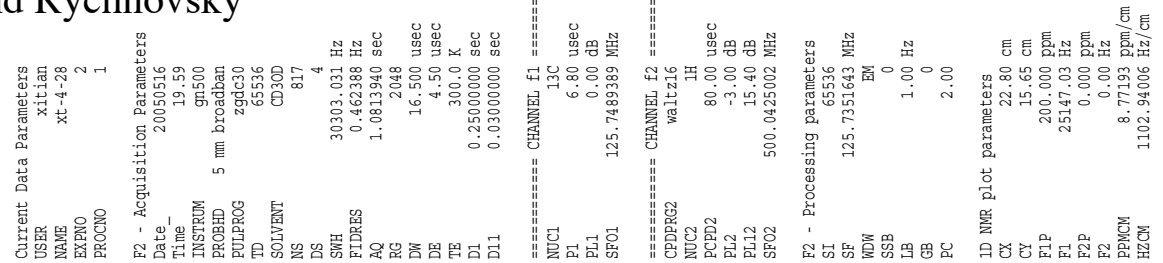

Page S-

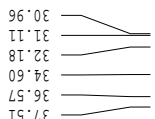

OZ. ५

$L \cdot 9 L$
$80 . L L$

$82 \cdot L L$

$\angle G \cdot L L=$
T0:6L

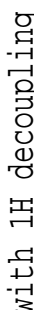

$80^{\circ} 0 \mathrm{II}$

66. $\mathrm{TZT} \longrightarrow$

60.081

$60^{\circ} \mathrm{LZT}$

TI. 627

$7 \tau \cdot 62 \mathrm{~T}$

$\tau \leftarrow \cdot 9 \varepsilon \mathrm{-}$

${ }_{9 G^{\circ}} \mathrm{T \hbar \tau}$

$\varepsilon \mathcal{L}^{\circ} \angle \nabla T=$
$\mp \hbar \cdot 8 \pi T=$

$\varpi 7 \cdot 8 \pi T$

$69.99 โ$

โ8.89โ-
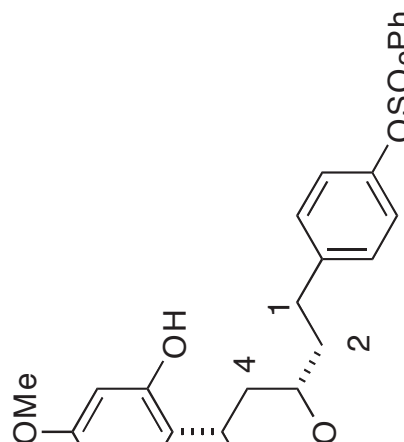<smiles>C1CCCCC1</smiles>

$\stackrel{a}{a}$

온

11
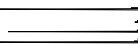

$-ㅇ$

$\overrightarrow{1}$

害

क्षे

西<smiles>[CH-]C(C)=CC</smiles>

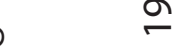

吾 
Tian, Jaber, and Rychnovsky

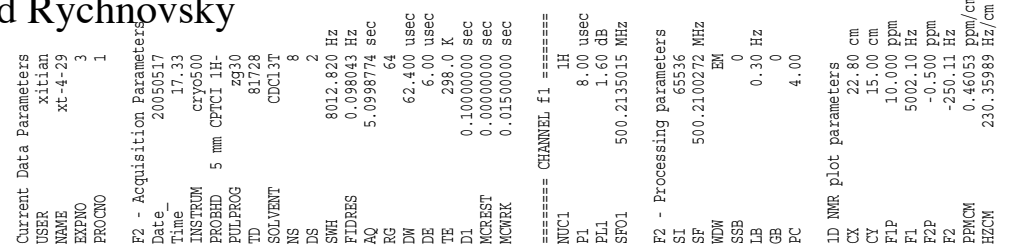

Page S-

40

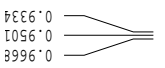

E899. โ

$9720 \cdot 0-\square$
$1600 \cdot 0 \longrightarrow$

$3996^{\circ} 0$

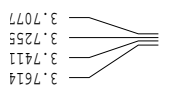

$396 \mathrm{~T} \cdot \mathrm{G}$
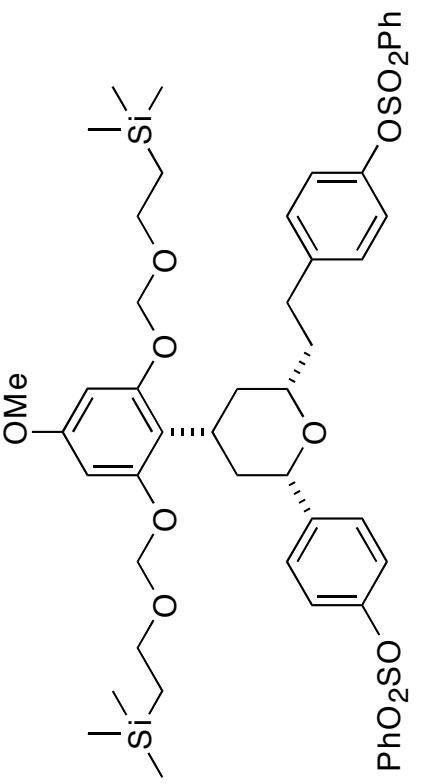

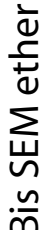

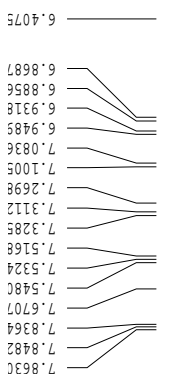

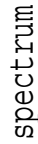


Tian, Jaber, and Rychnovsky

Page S-

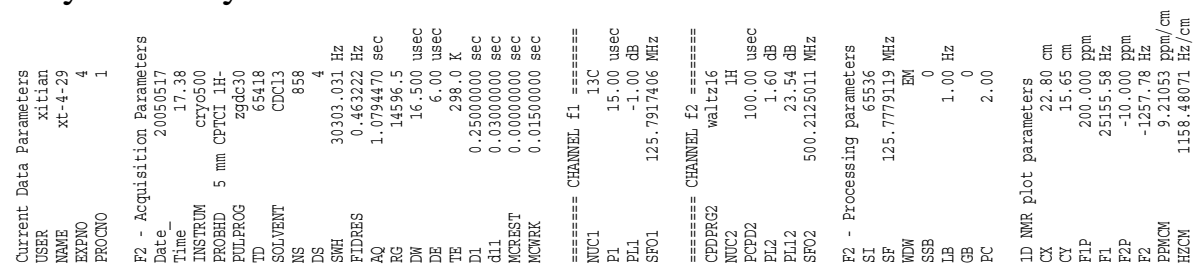

$00 \cdot \mathrm{T}-\mathrm{C}$
$00 \cdot 0$

$\varepsilon 0^{\circ} 8 \mathrm{~T}$

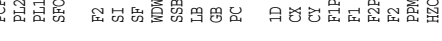

$\hbar \tau^{\circ} \tau \varepsilon$
$8 \varepsilon \cdot \tau \varepsilon$
$60 \cdot 9 \varepsilon$
$\tau L^{\circ} \cdot 9 \varepsilon$
$\varepsilon 8^{\circ} \cdot \varepsilon \varepsilon$

૪६૬૬

งะ'99

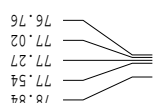

$\angle 6^{\circ} \pm 6$

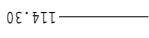

66. 2 IZT

$86^{\circ} .92 \mathrm{-}$

$05^{\circ} 82 \mathrm{~T}$

$80^{\circ} 62 \mathrm{~T} \longrightarrow$

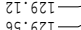

9. $62 \mathrm{IT}$

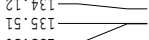

9S. SET-

$2 g^{\circ} \tau \hbar \tau-$
$6 \varepsilon \cdot 2 \tau \tau-$

$95^{\circ} \angle D T$
$9 \sigma^{\circ} 8 \pi T$

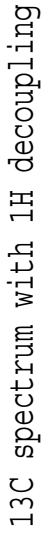

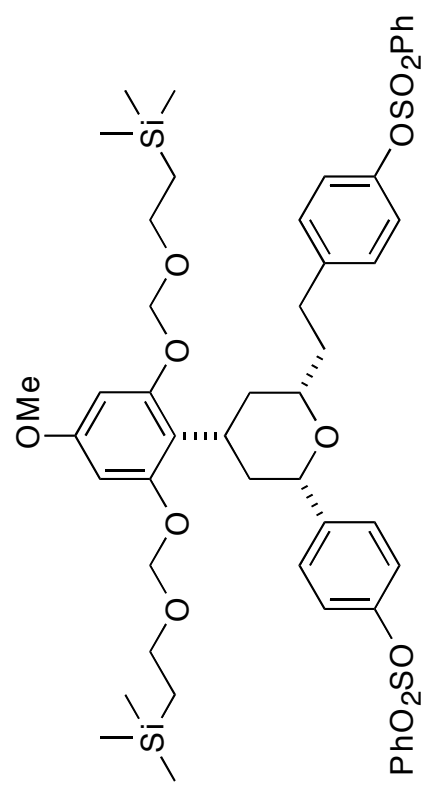

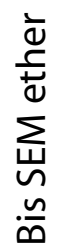

$8 T^{\circ} 69 \mathrm{I}$

$-\infty$

움 
Tian, Jaber, and Rychnovsky

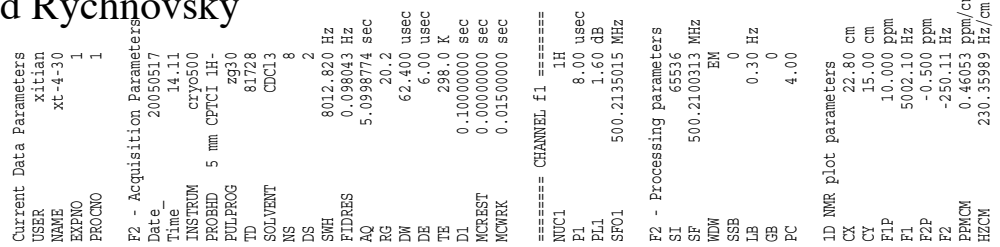

Page S-

42

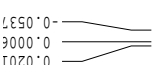

$\checkmark 906^{\circ} 0$

$\square \varepsilon 76^{\circ} \circ$
$\angle 8 \varepsilon 6^{\circ} \circ$

$3896^{\circ} 0$

$\$ 986^{\circ} \cdot 0$

6089. โ

$\sigma \tau 89^{\circ} \varepsilon$
$7989^{\circ} \varepsilon$

$3000^{\circ} \varepsilon$

$\exists \neg T L \cdot \varepsilon \longrightarrow$

โ6TL' $\varepsilon$

วโร $8^{\circ} \varepsilon$

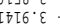

$3626^{\circ} \varepsilon$

โร80. 5

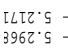

$\operatorname{L7} 99.9$
I958.9

$[958.9$
[عL8.9

3626.9

2976.9

$\varepsilon \angle 0^{\circ} \mathrm{L}$

3298.2

รTI $: L$

T909. $\mathrm{L}$
$20 \mathrm{TS}^{\circ} \mathrm{L}$

ZOTS. $L$

$3929^{\circ} L$

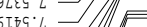

$1679^{\circ} \mathrm{L}$

э299. L

政

5 TZ8.

$\exists\left\llcorner\varepsilon 8^{\circ} \mathrm{L}-\right.$

$\square 98^{\circ} \mathrm{L}$

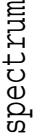

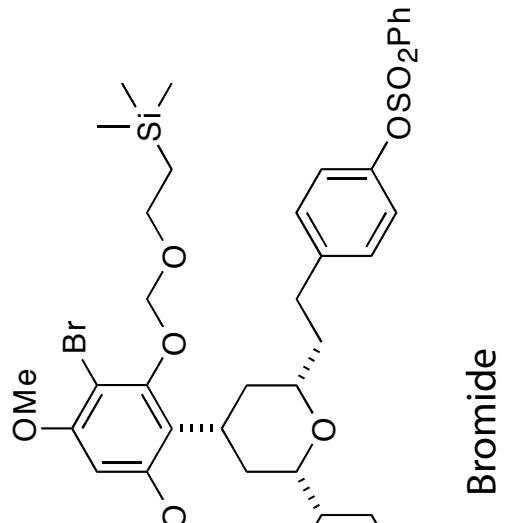

$\frac{\frac{d}{0}}{\frac{8}{0}}$

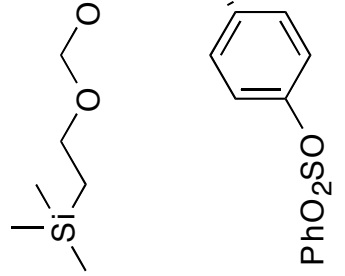

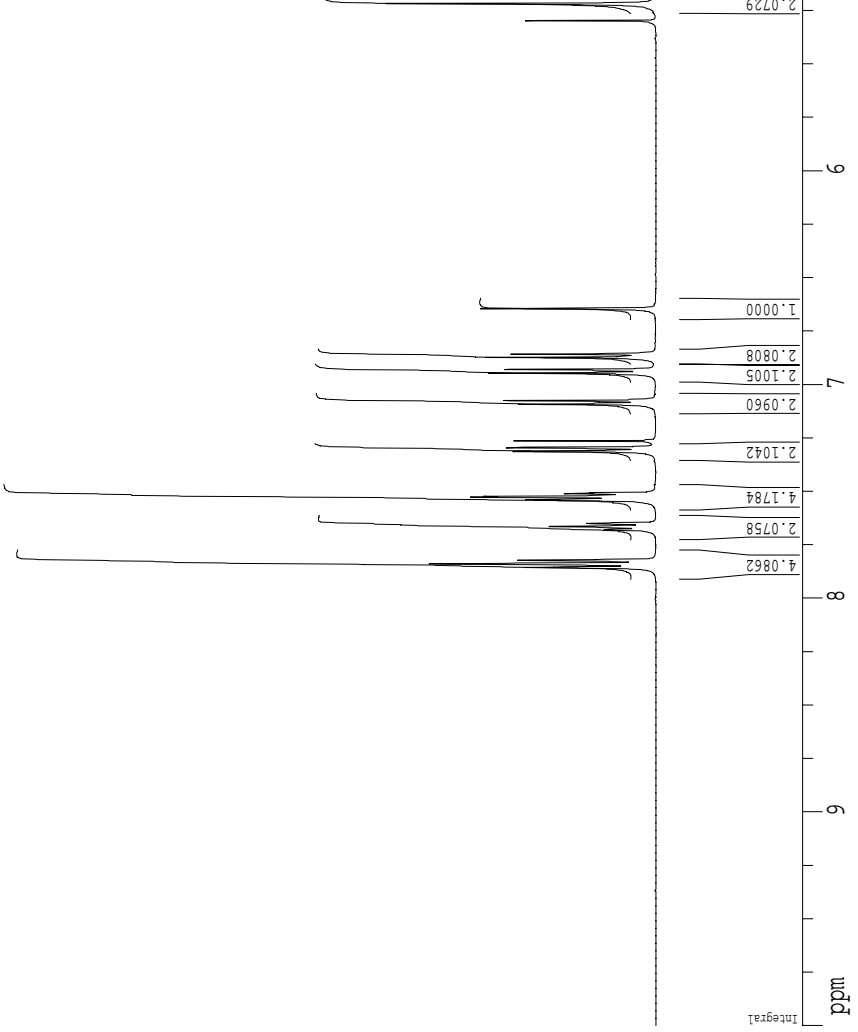


Tian, Jaber, and Rychnovsky

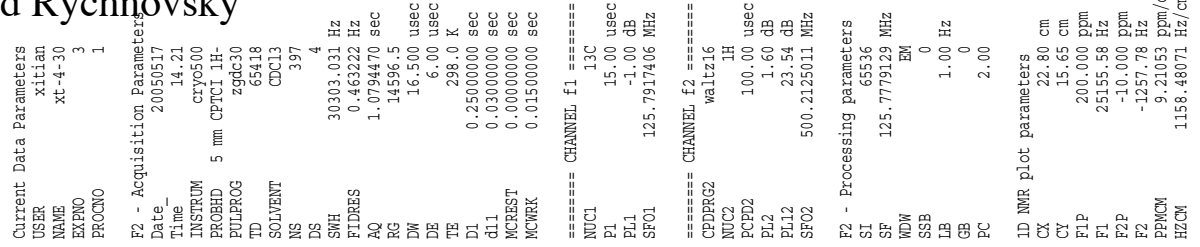

Page S-

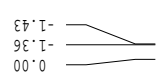

${ }_{0 \varepsilon^{\circ} 8 \tau} 8 \tau=$

$\tau \varepsilon \cdot \tau \varepsilon$
$\varepsilon \varepsilon^{\circ} \ni \varepsilon$
$L 6^{\circ} \ni \varepsilon$
$89^{\circ} 9 \varepsilon$
$88^{\circ} L \varepsilon$

$6 \varepsilon \cdot 99$

$\varepsilon \varsigma^{\circ} .99$
T0.89

$L L \cdot 9 L$
$\varepsilon 0^{\circ} L L$
$8 L^{\circ} L L L$
$\varepsilon 8^{\circ} 8 L$<smiles>CCOCCC(C)(C)OCCO[GeH3]</smiles><smiles>[2H]c1c([18OH])cc(O)c([C@@H]2C[C@H](CC)O[C@H](CC)C2)c1OC</smiles>

IL'26

$\varepsilon \varepsilon^{*} 96$

$97.66=$

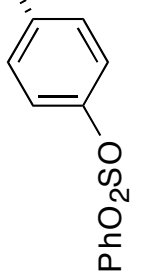

$\frac{\frac{0}{0}}{\frac{0}{0}}$

$99 \cdot 0 Z \mathrm{I}$

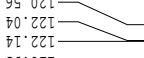

$86^{\circ} 92 \mathrm{~T}$

$80^{\circ} 8 \mathrm{ZT}-$
$80^{\circ} 6 \mathrm{~T}-$

$\varepsilon \tau^{\circ} 62 \tau \longrightarrow$

$8 \mathrm{I}^{\circ} \cdot 62 \mathrm{~T}$

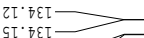

Gโ'

हร. ระโ-

It. $\tau \hbar \tau$

$69^{\circ} \angle \pi T-$
$09^{\circ} \cdot 875$

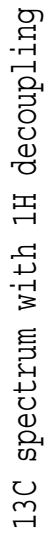

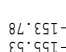

६ร. '.

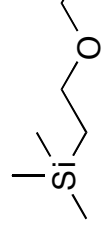

足

듬 


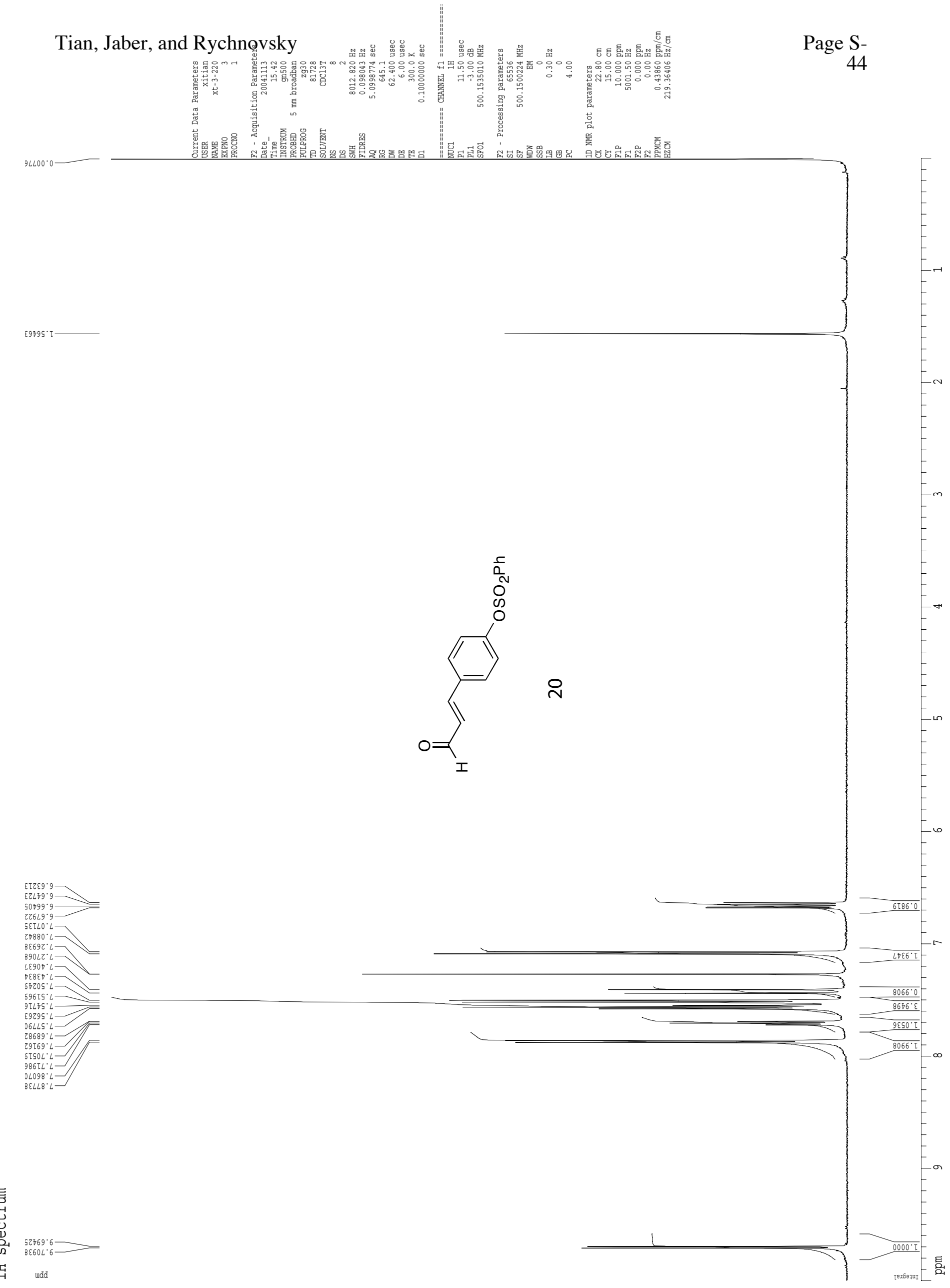




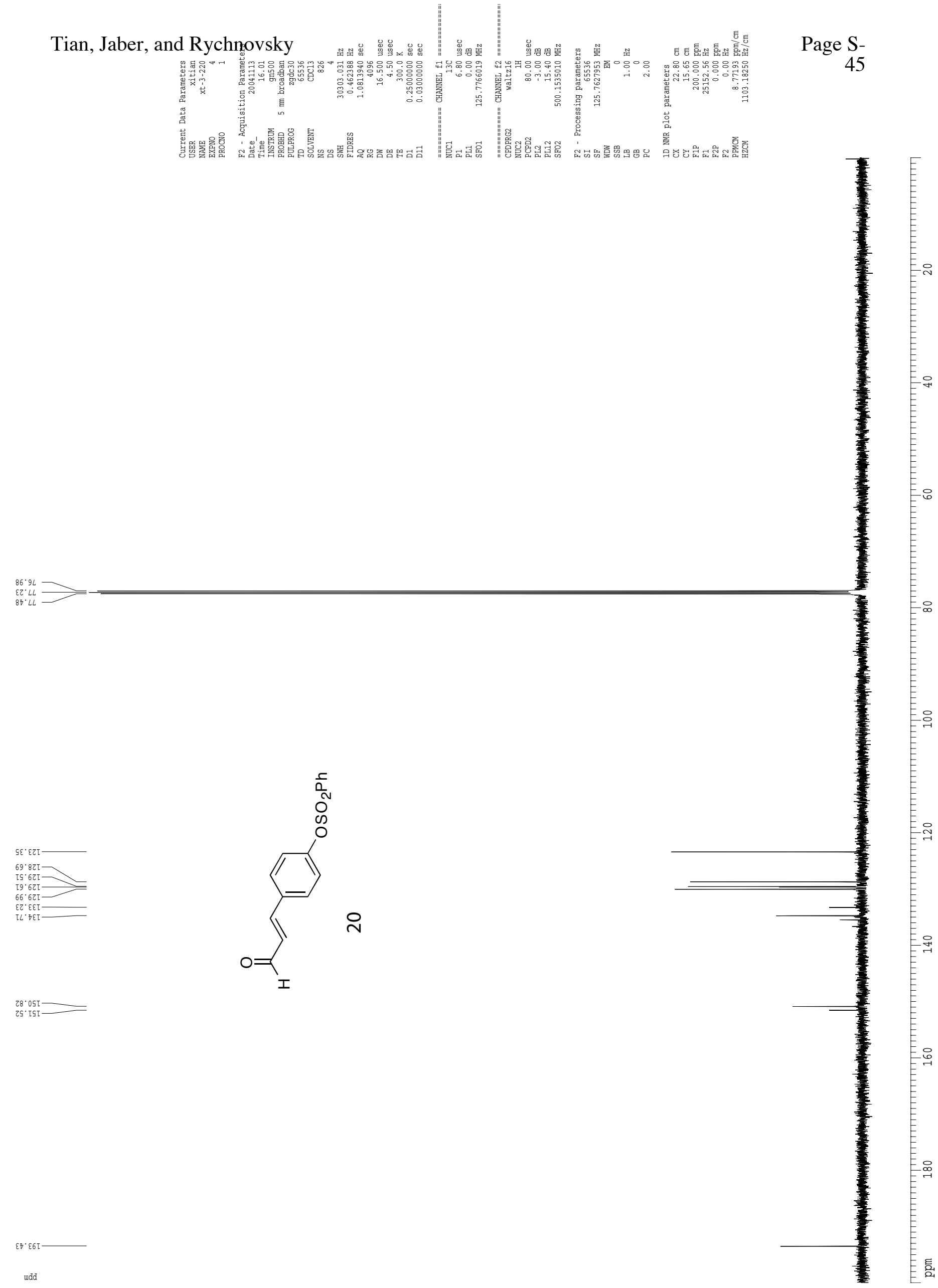


Tian, Jaber, and Rychnovsky

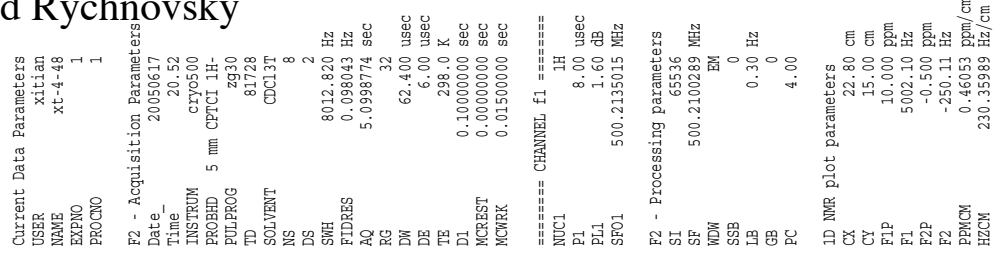

Page S-

46

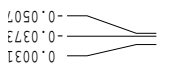

$3998^{\circ}$
$1288^{\circ}$

$3006^{\circ} 0$

$2926^{\circ} \circ$
$3 \mathrm{t} 76^{\circ} \circ$

$3 \mathrm{t} t 6^{\circ} 0$
$2696^{\circ} 0$

$\left[\angle 8 \varsigma^{\circ} \cdot \tau\right.$

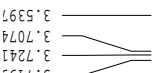

$[\succsim \tau L \cdot \varepsilon=$

$668 \varepsilon^{\circ}$

$\angle L 06^{\circ} \div$

โร9น.
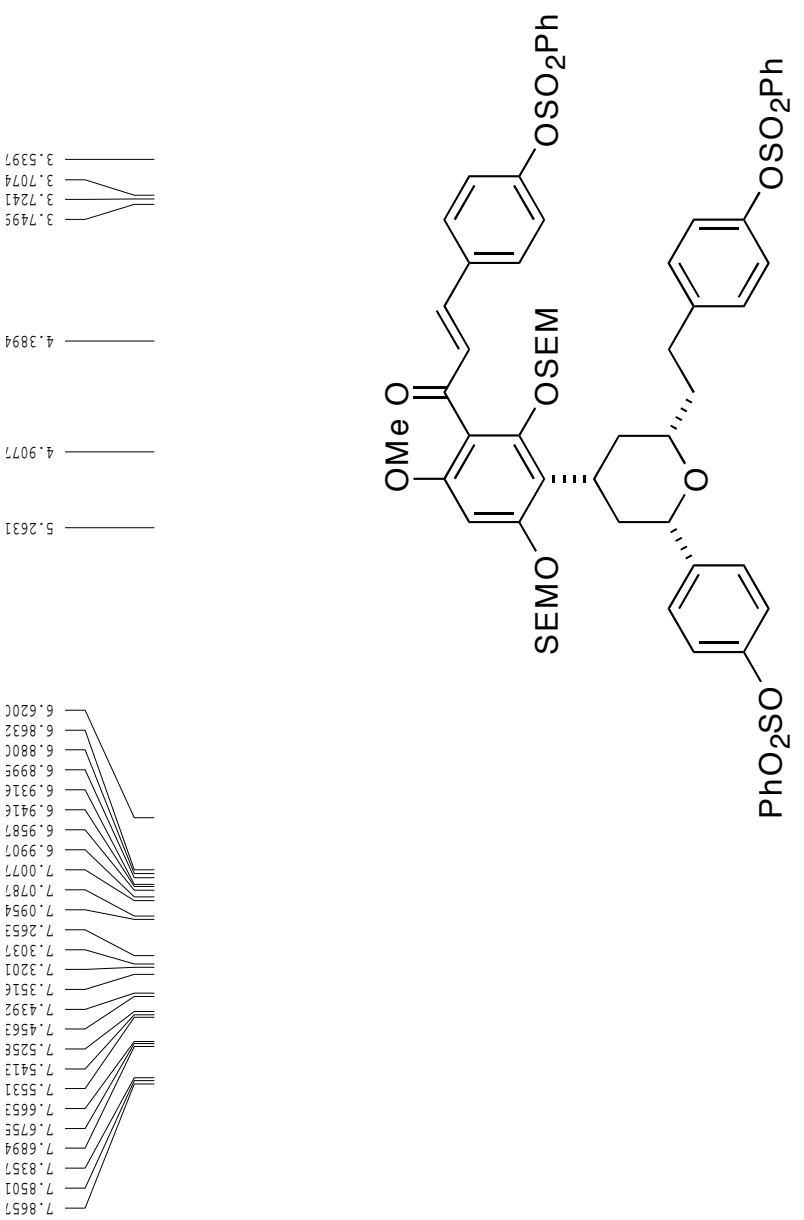

$\bar{\sim}$

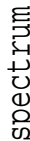

న్ 
Tian, Jaber, and Rychnovsky
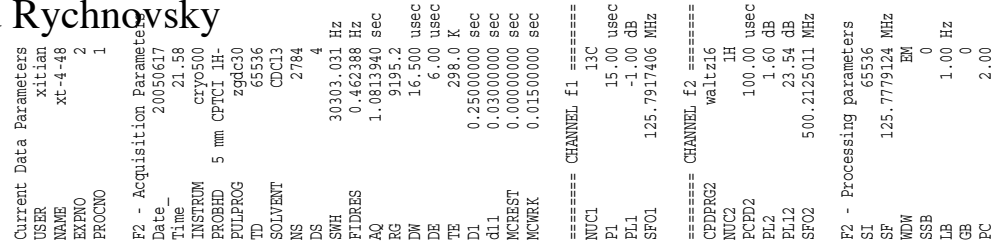

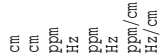

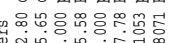
宸 救

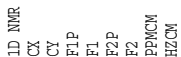

Page S-

47

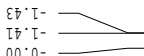

${ }_{91}^{00} 8 \mathrm{8T}=$

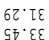

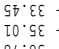

$8 L^{\circ} 9 \varepsilon=\square$
$\angle 8^{\circ} L \varepsilon=$

96.99

99.99
78.69

$L L \cdot 9 L \square$
$20 \cdot L L=$
$82 \cdot L L=$
$0 L \cdot L L=$
$\varepsilon 8 \cdot 8 L=$

$\mp 9^{\circ} 26$

วะ' 96

$\varepsilon 6.66$

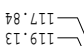

$90^{\circ} 28 \mathrm{TT}$

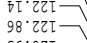

$\varepsilon \sigma^{\circ} \cdot 92 \mathrm{TT}$

$80^{\circ} \cdot 8 \mathrm{CIT}$
$\angle \mathrm{L} \cdot \mathrm{T}$

$60.62 \mathrm{~T}$

ร2. $62 \mathrm{I}$

$9 \varepsilon^{\circ} 62 \mathrm{I}$

$6 \sigma^{\circ} \cdot 6 \mathrm{ZT} \longrightarrow$

$\varepsilon 8 \cdot \varepsilon \varepsilon \tau-$

$\varepsilon \tau \cdot \ni \varepsilon T-$

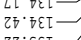

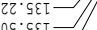

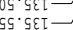

O๐. โIT-

ह0. $2 \nabla T-$

O) $65 . \angle E T-$

65. $\angle E T-$

ने

ठे

虫

虹

E

亮

这

户.

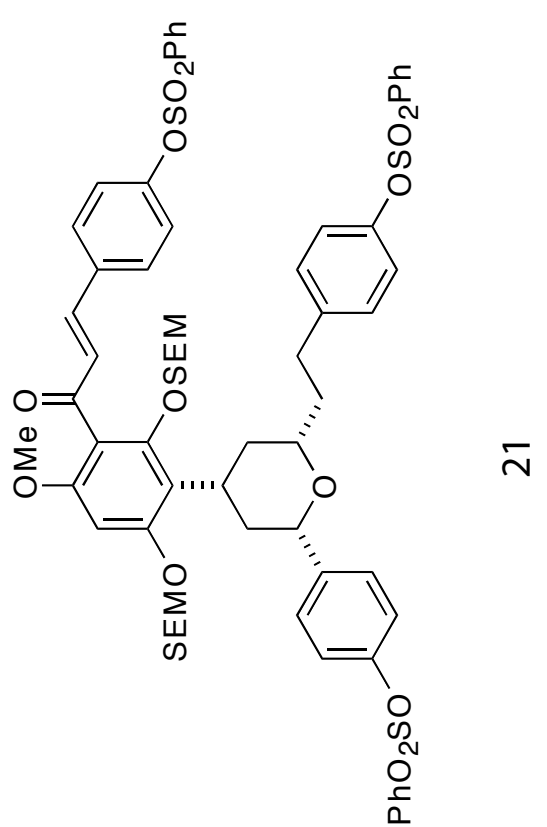


Tian, Jaber, and Rychnovsky

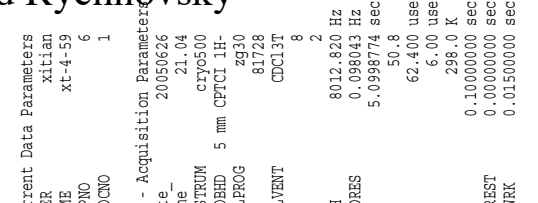

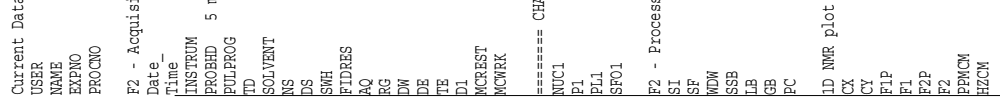

E9T92 โ

bZTSO $: 2$

$59667^{\circ} \varepsilon$

โ98โ8. $\varepsilon$

$\llcorner 5662 \cdot s$

$59 L 98^{\circ} 9$

$\exists z 29)^{\circ} L$

IETOS:

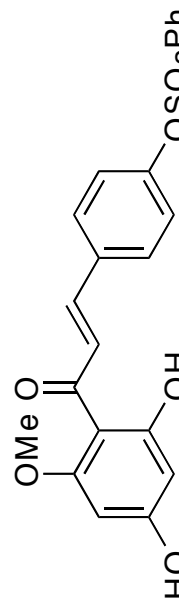

Page S-

48

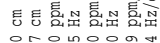

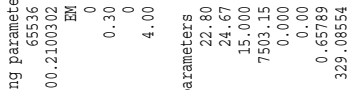


Tian, Jaber, and Rychnovsky
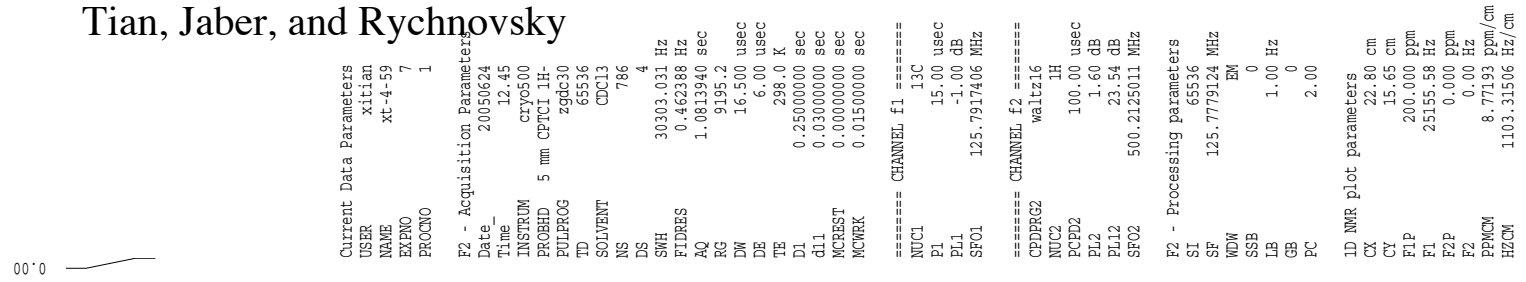

Page S-

$6 T^{\circ} \mathrm{t}$

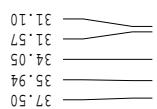

$9 L \cdot \varsigma \varsigma$

25.09

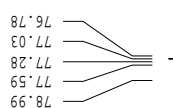

ธน' $\tau 6$
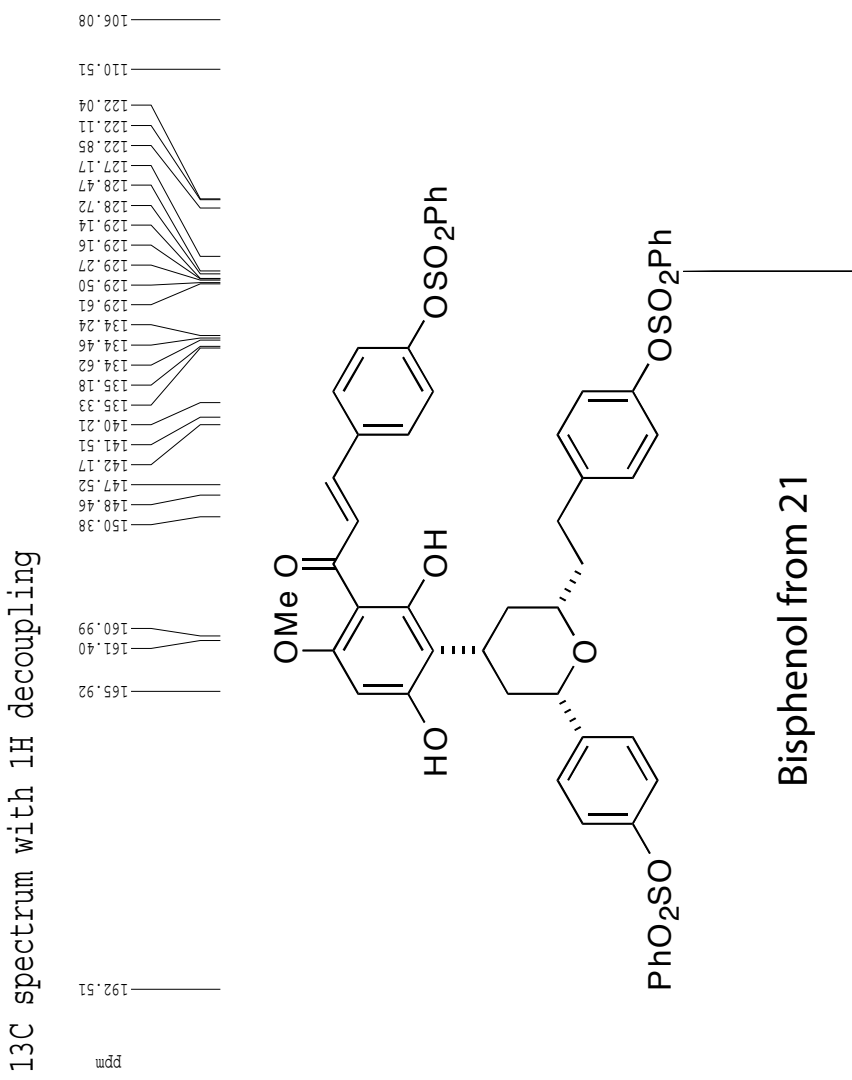
Tian, Jaber, and Rychnovsky

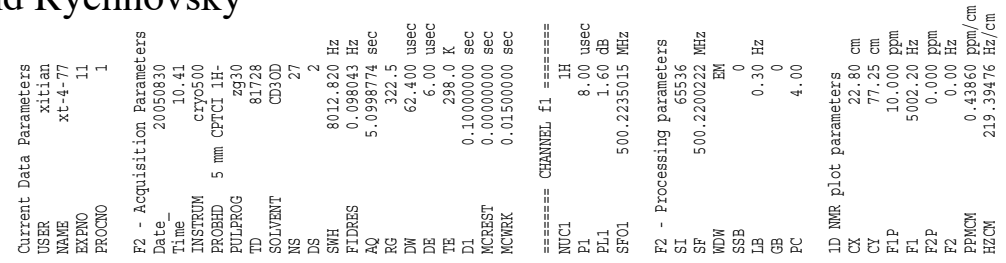

Page S-

50

$\angle L F 0 \varepsilon \cdot \varepsilon$

$\varepsilon 9 L 0 \varepsilon^{\cdot} \varepsilon-$

$92699^{\circ} \varepsilon$

$92668 \cdot \varepsilon$

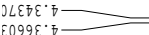

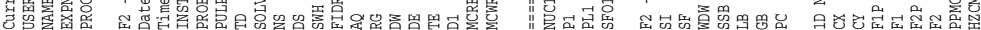

$56698^{\circ}+$

BยЕโ0.9-

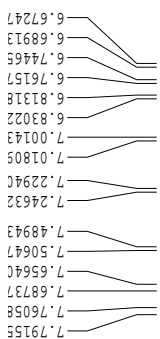

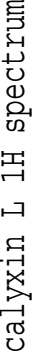

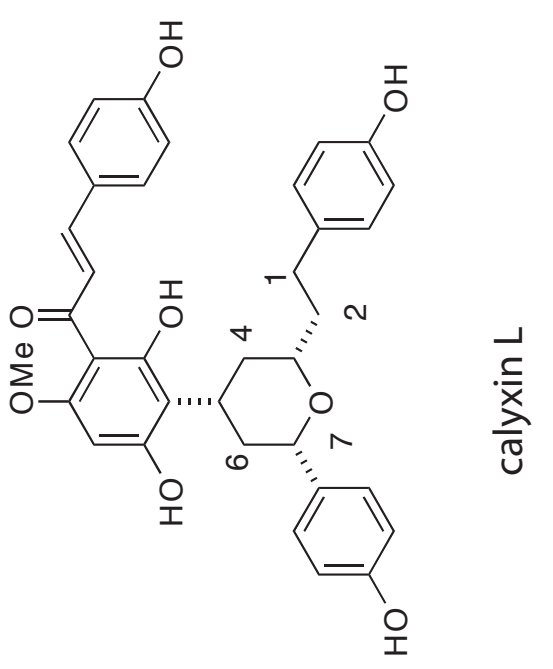

$\frac{\sqrt{c}}{\frac{1}{\sqrt{0}}}$
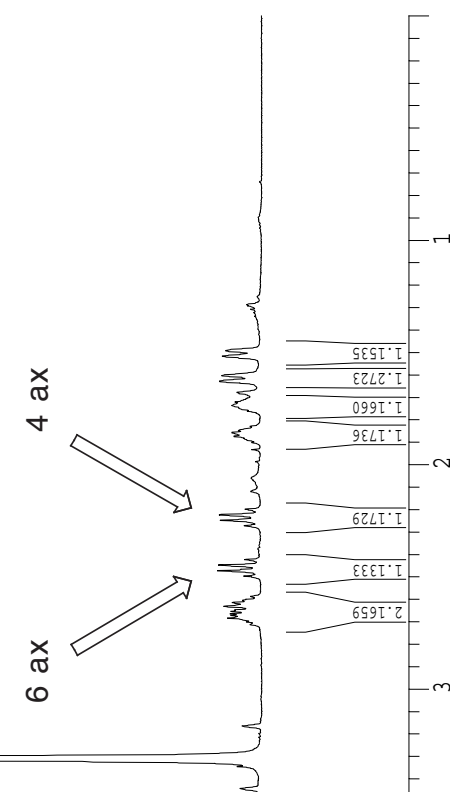

$6 Z \tau^{\prime} \tau$

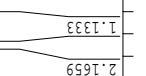

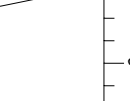

$96 \tau^{\circ} \tau_{-}^{-}$

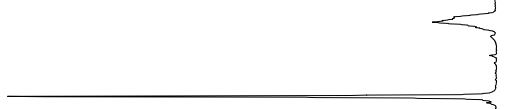

$8 \mathrm{I \varepsilon Z^{ \circ } \varepsilon}$

$N$
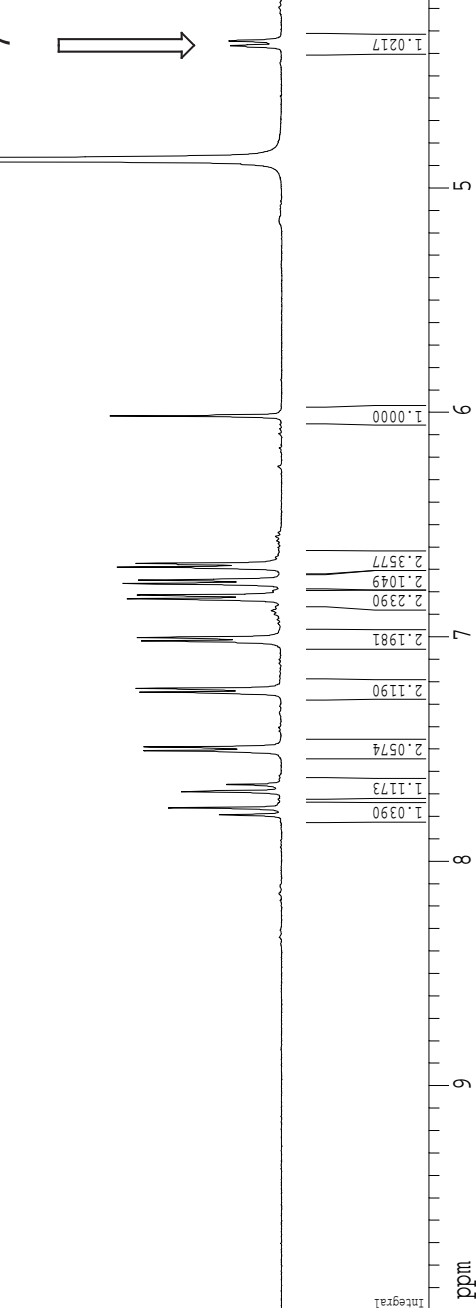
Tian, Jaber, and Rychnovsky

Page S-
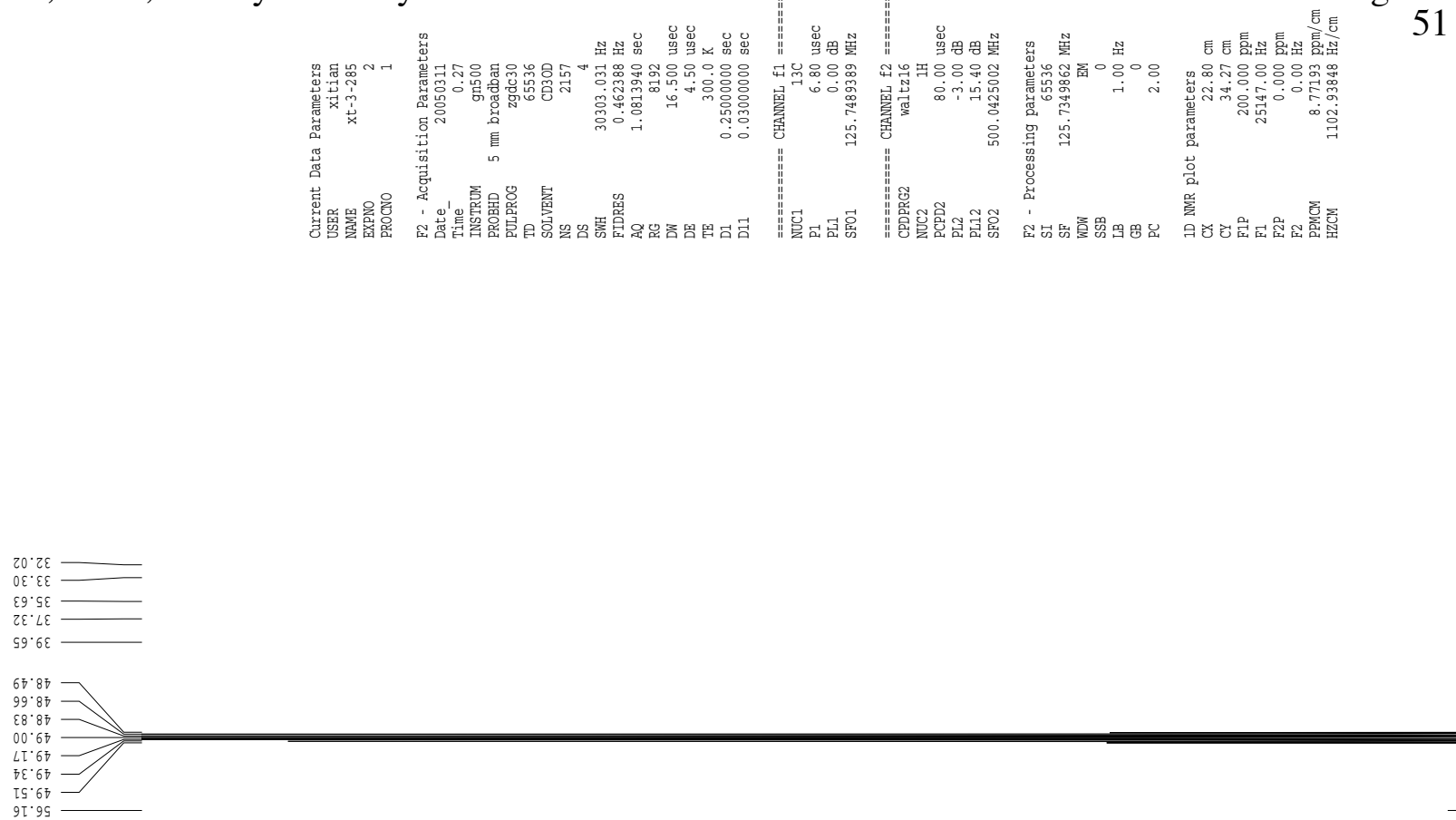

$\angle F^{\circ} \cdot 6 L$
$59 \cdot 18$

II' 2

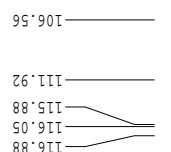

S0.9IT-
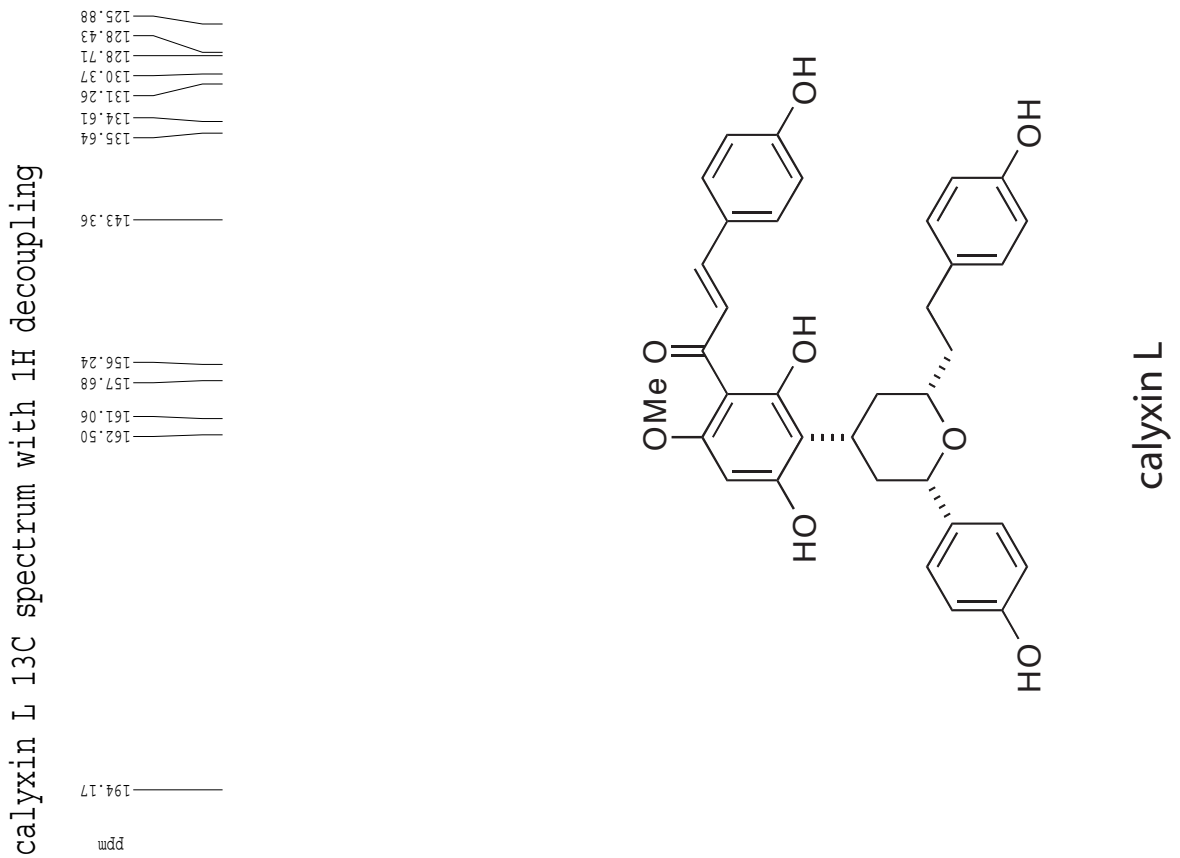
Tian, Jaber, and Rychnovsky

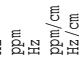

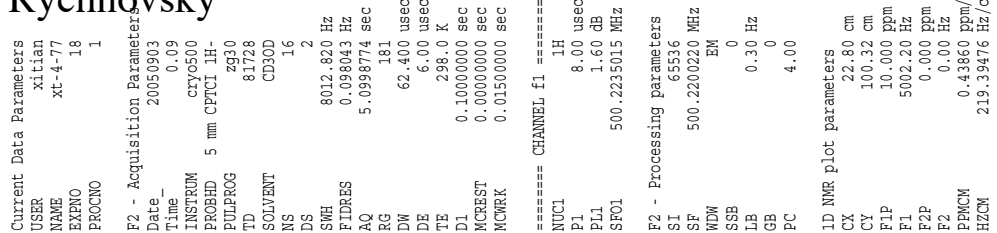

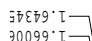

2T5L9.

$\left.30889^{\circ}\right]$

B8E0L.
ETgLL

LITEL โ

jहST8.

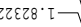

$16628^{\circ} \mathrm{\tau}$

э. $\angle 98^{\circ} \mathrm{T}$

$298 \angle 8^{\circ} \mathrm{I}-$

$39888^{\circ} \mathrm{T}-$

${ }^{3} \varepsilon 906^{\circ}$ โ

$\angle 01 \varepsilon 6^{\circ}$

$6 \varepsilon 076^{\circ} \mathrm{T}$
$\forall \angle 866^{\circ} \mathrm{T}-$

$\square L 866^{\circ} \mathrm{T}-$
$\mathrm{L} \angle 000^{\circ} \mathrm{Z}$

$29970^{\circ} z$

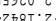

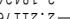

$79995 \cdot 7$

รTOLS: 2

59SLG'Z-

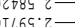

$5 \% 209 \cdot 2=$

$509 \tau 9 \cdot 2-$

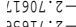

${ }^{3} 9 \mathrm{TSZL} L \cdot 2-$

$\operatorname{cosel} z$

โEZSL' 2

¿टध9L:

$16 \mathrm{TIT} \cdot \varepsilon-$

ว96 $6 \mathrm{Tt} \cdot \varepsilon-$

उहLZT' $\varepsilon-$

¿ $670 \varepsilon^{\circ} \varepsilon$

$\triangle L \varepsilon \mp \varepsilon \cdot \varepsilon-1$

$6858 \mathrm{~L} \cdot \varepsilon$

$38698^{\circ} \mathrm{F}$

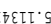

ต89ยt's

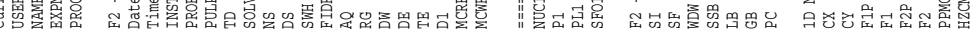

.
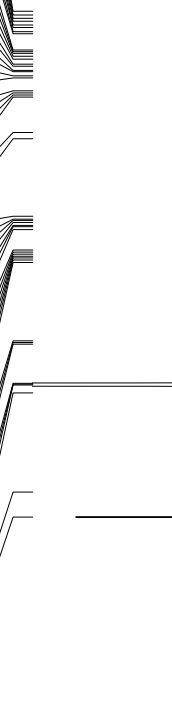

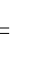

$36 \mp \varepsilon 0.9$
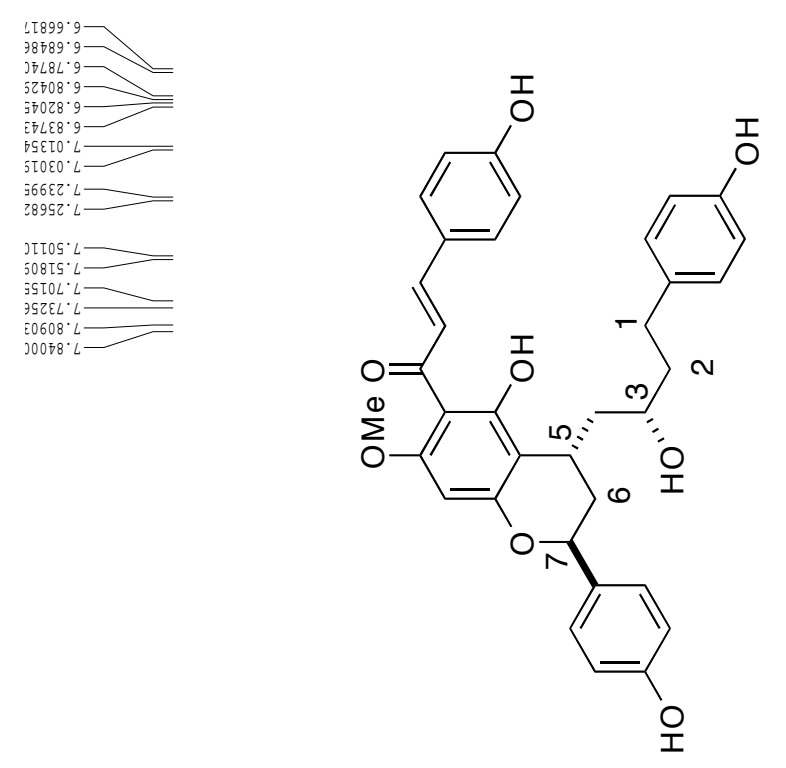

$\frac{\text { 衣 }}{\frac{\sqrt{x}}{\sqrt[0]{0}}}$
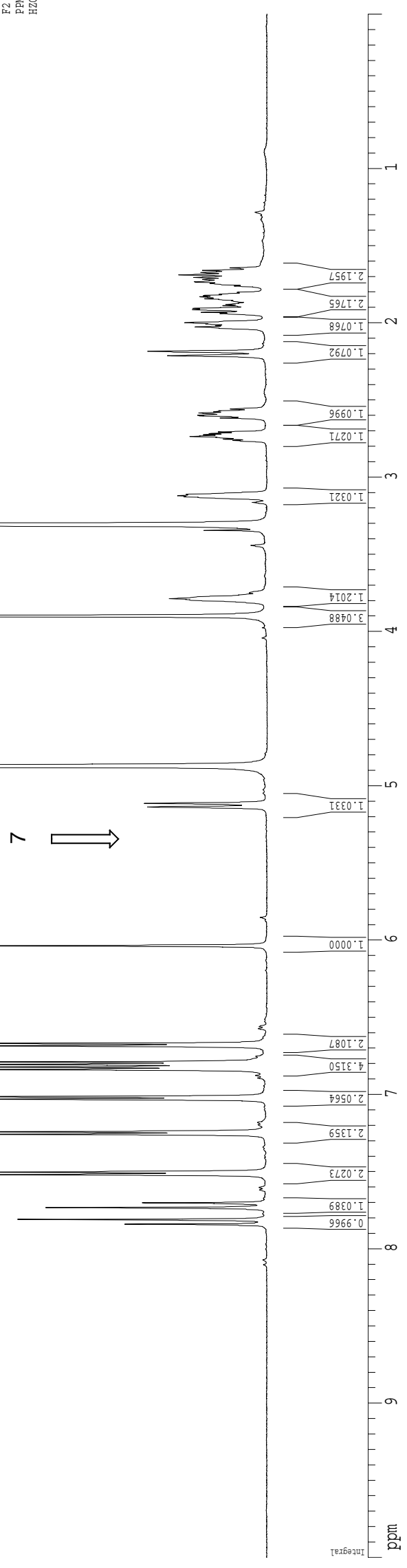
Tian, Jaber, and Rychnovsky

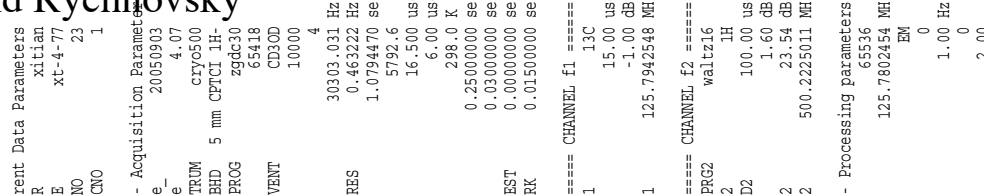

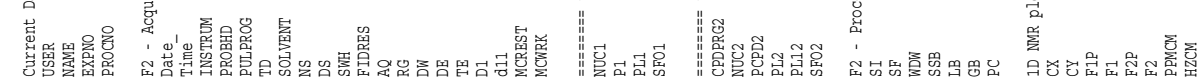

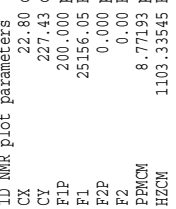

Page S-

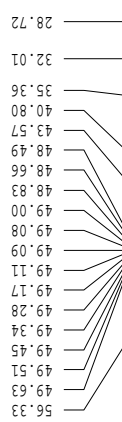

$8 \varepsilon^{\circ} \tau L$ $\angle 8 \cdot S L$

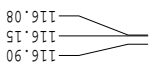

IS. SRT

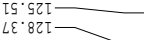

$0 L \cdot 8 Z \tau$

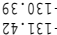

$\tau\rceil \cdot \varepsilon \varepsilon T$

و૬.

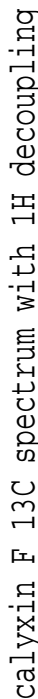

$66^{\circ} \varepsilon \mp \tau-$

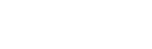

$67 \cdot 99 \tau$

S๐. 89 โ

ว2. 19 -

$\angle 8 \cdot 29 \tau-$
$28.29 \tau-$

$02 \cdot 99$ 工

巨్

.

,

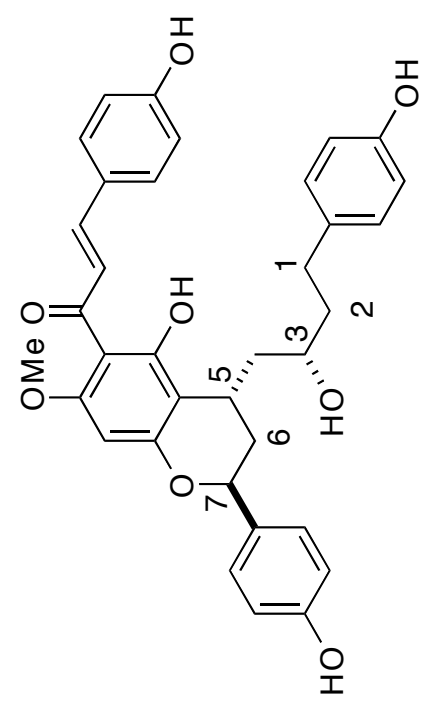

$\frac{\frac{1}{x}}{\frac{\pi}{\sqrt{U}}}$
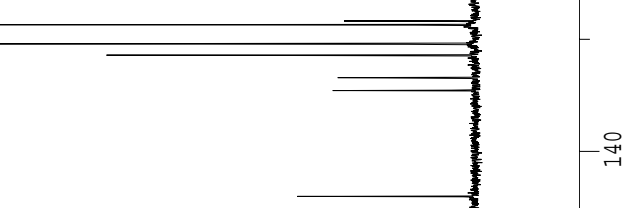

$$
\frac{\sqrt{x}}{\sqrt[0]{0}}
$$


Tian, Jaber, and Rychnovsky

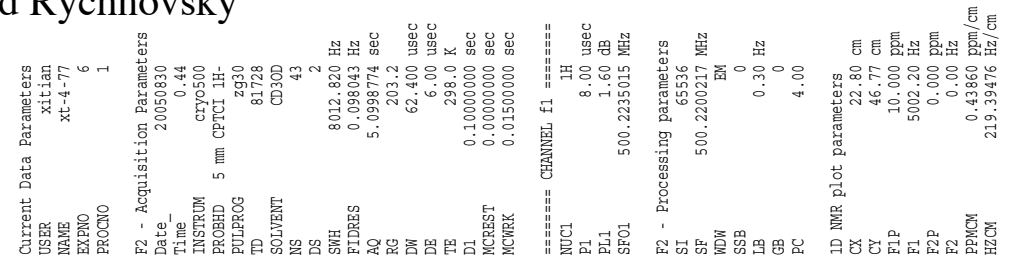

Page S-

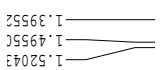

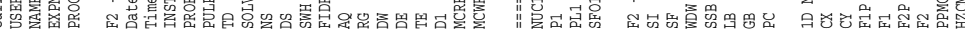

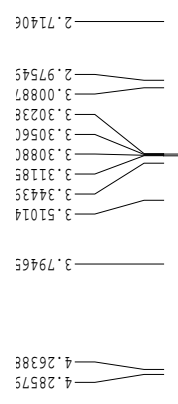

JTOL8. ถ

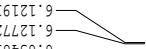

$32 L 2 T \cdot 9$
L $979 \cdot 9$

[7099.9

[ZLL 2 โ69.9]

โISZL. 9

j920L.9-

โ5858.9-

¡S $58.9-$

$3 £ 676.9-$

$666966^{\circ} \cdot 9-$

$\triangle L E \nabla 0^{\circ} \mathrm{L}-$

$39090^{\circ} \mathrm{L}-$

जTgET:L

至 $\left.\quad 3 \varepsilon 89 \varepsilon^{\circ} \circ\right]$

บ

$\sum_{-1}^{-1}$

임

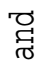

$\Sigma$

-

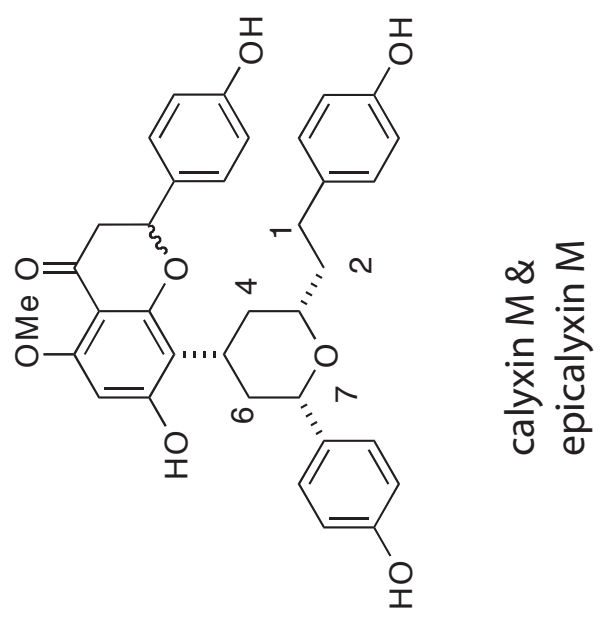


Tian, Jaber, and Rychnovsky

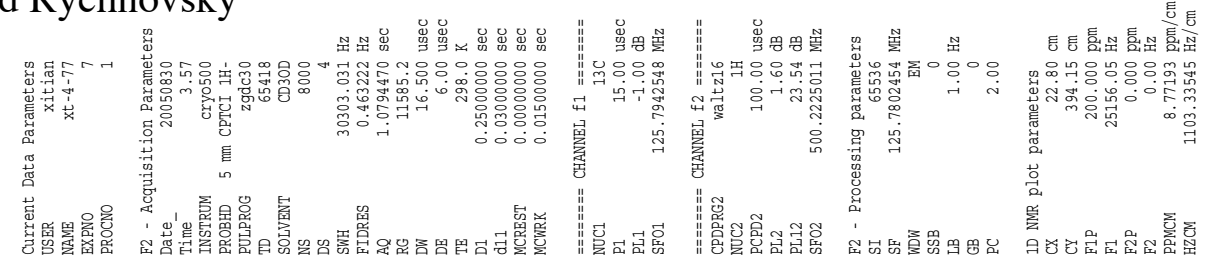

Page S-

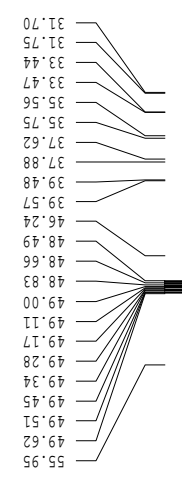

$2 \tau \cdot 6 L$

80.08
$0 \varepsilon^{\circ} .08$

${ }_{\varepsilon 6}^{78 \cdot \varepsilon 6} \longrightarrow$

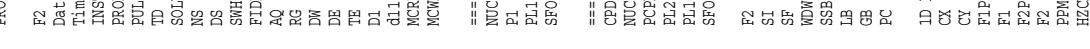
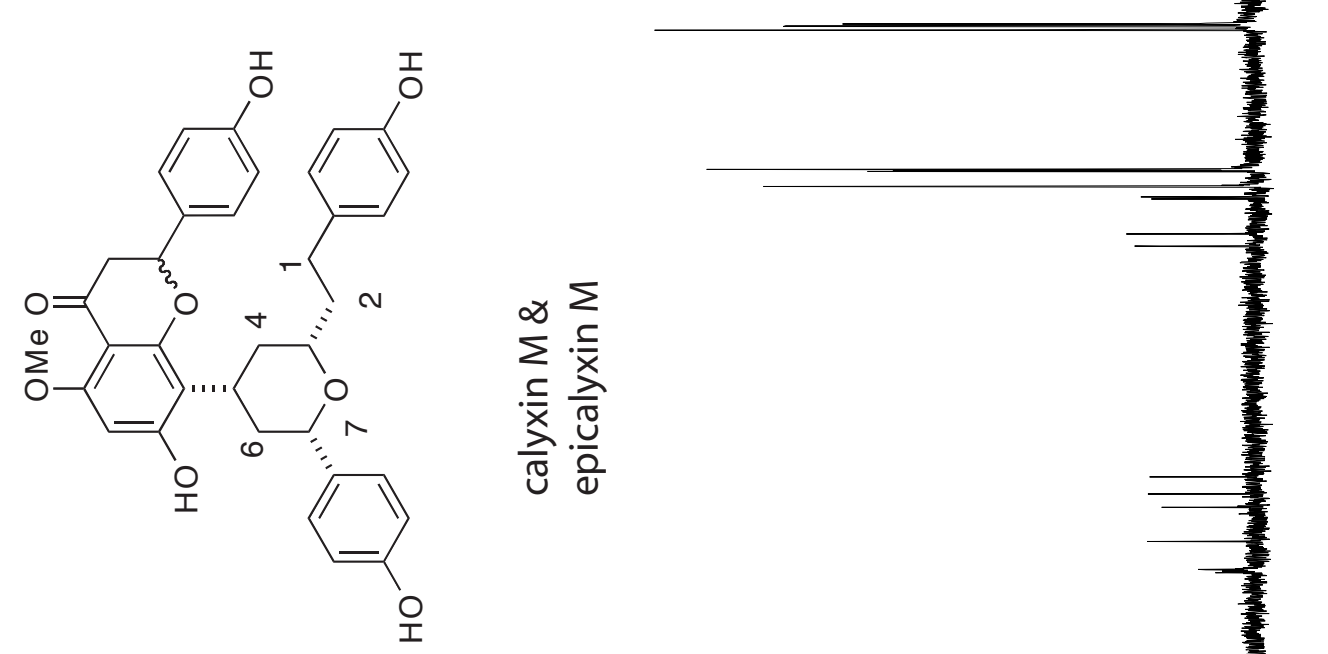
Tian, Jaber, and Rychnovsky

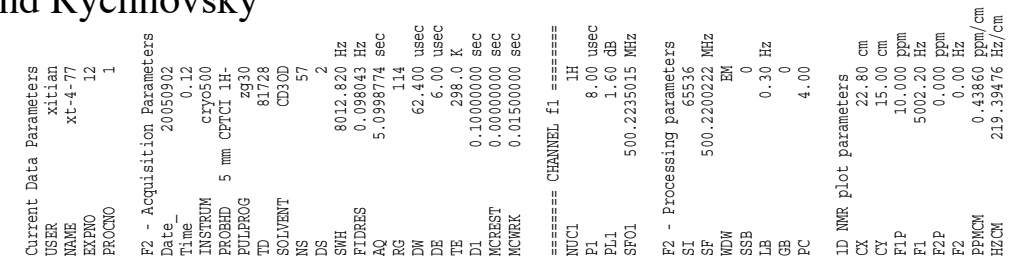

Page S-

56

E9T0\&: $\varepsilon$

$3970 \varepsilon^{\circ} \varepsilon-$
$\left[9 L O \varepsilon^{\prime} \varepsilon\right.$

${ }_{396 L L} \varepsilon-$

(n)

$600 \angle 8 \cdot 7$

$30<2 \tau \cdot 9$

$36779 \cdot 9$

$30 \varepsilon L L \cdot 9$
$5868 L$

$69508 \cdot 9$

¿L608.9

$39928 \cdot 9$

IL988.9-

26298.9

$39098.9-$

$365 \mathrm{TZ} \cdot$

ELgER L

Эड9वह:L-

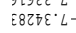

言

E8Z9\&:L-

,

ठ․

可

0

-

움

ఠ

C

点

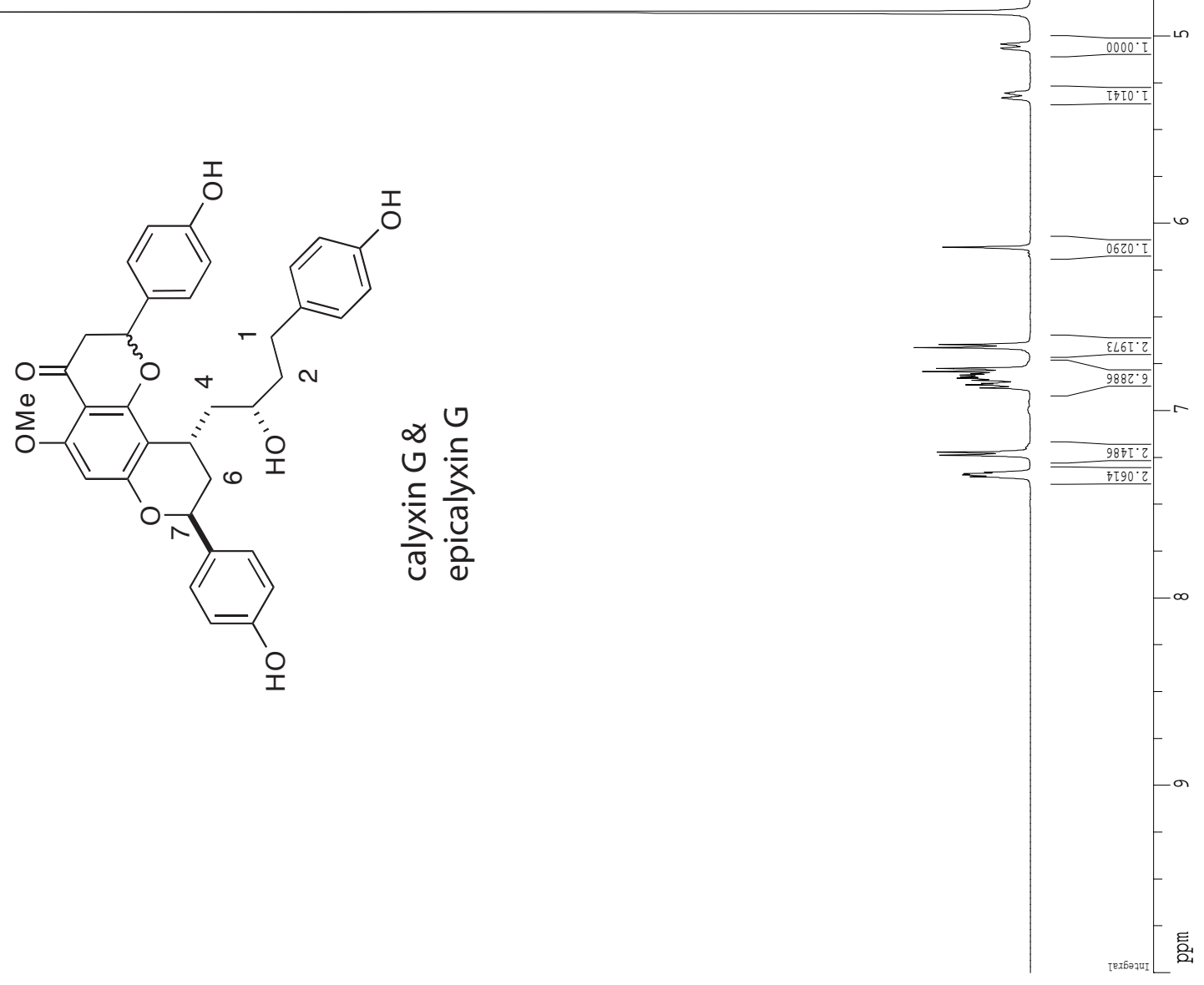


Tian, Jaber, and Rychnovsky

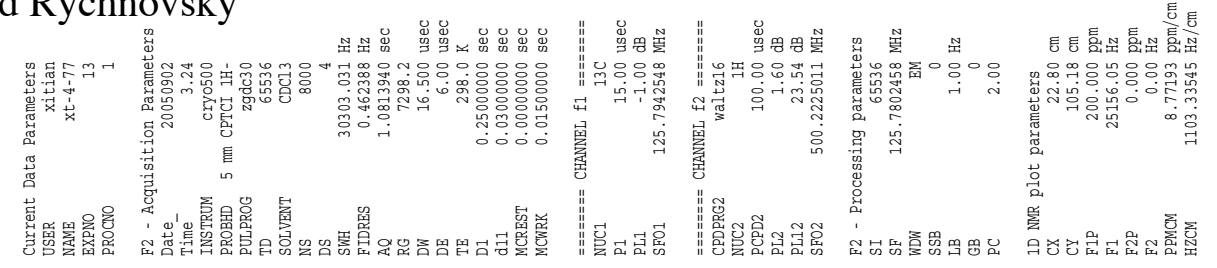

Page S-

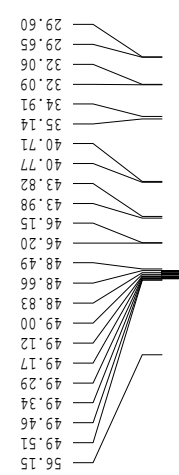

$6 L \cdot T L=$

$+8 \cdot 24$

I8. ${ }^{\circ}{ }^{\circ} \mathrm{GL} \longrightarrow$

TL.08
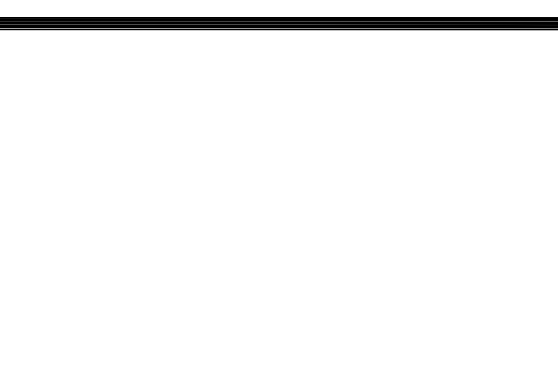

$\varepsilon 9^{*} \cdot 6$
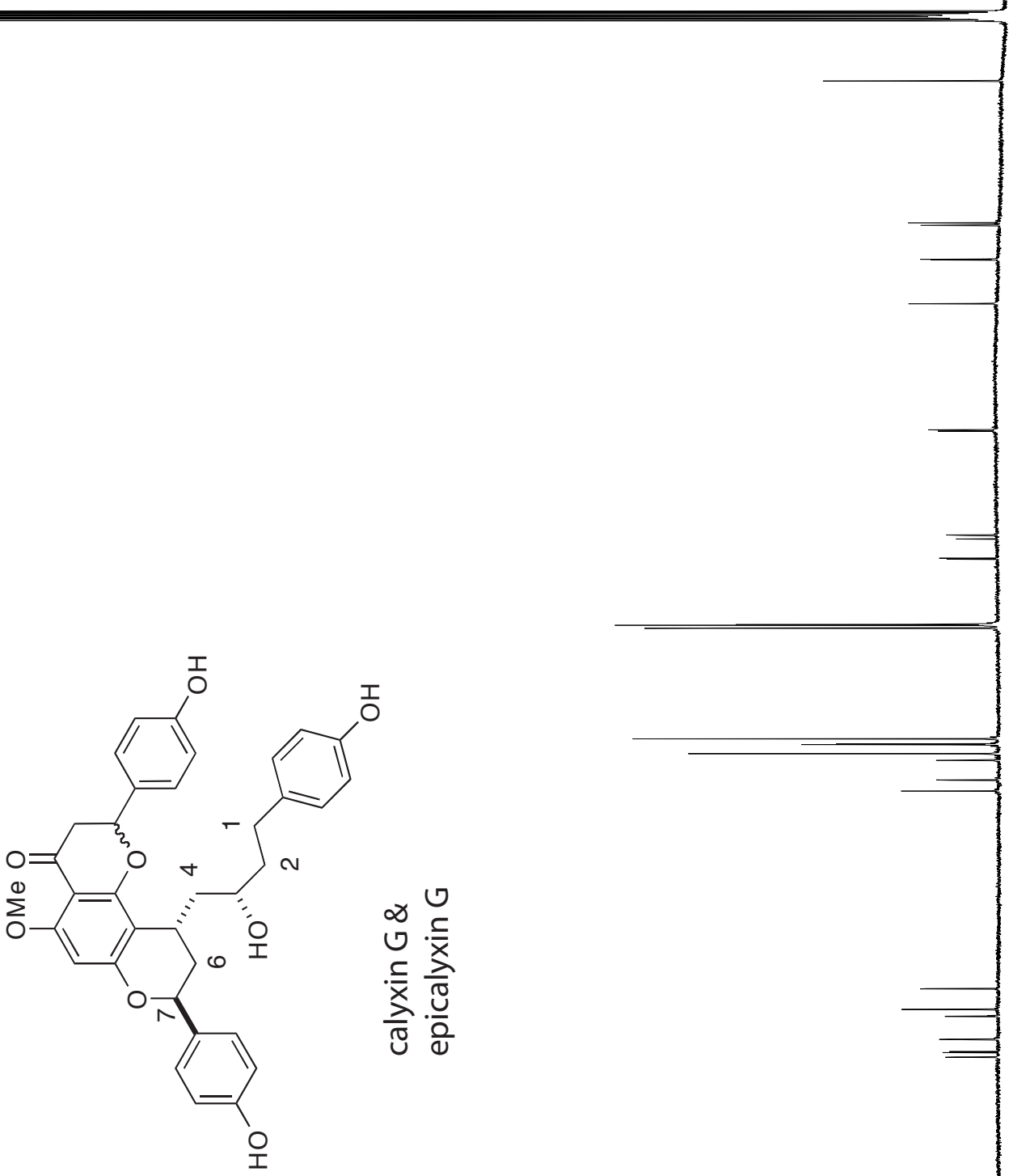

$\longrightarrow$

${ }_{72}^{27 \cdot 900-}=$ SL: 807
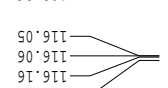

$$
\text { 9I. } 9 \tau \mathrm{9t}=
$$

$89 \cdot 8 Z \mathrm{~T}$
$Z Z \cdot 6 Z \mathrm{~L}$

$27 \cdot 62 \mathrm{I}$
$0 \varepsilon \cdot 62 \mathrm{I}$

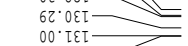

$90^{\circ} \tau \varepsilon I=$
$\mathrm{LI} \cdot \varepsilon \varepsilon \mathrm{Z} I=$

$\tau \tau^{\prime} \ni \varepsilon \tau$

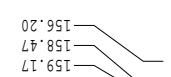

LI'6ST

7L.'T $\longrightarrow$

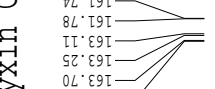

空

$8 L^{\circ} \varepsilon 9 \mathrm{~L}$

ช 
Tian, Jaber, and Rychnovsky

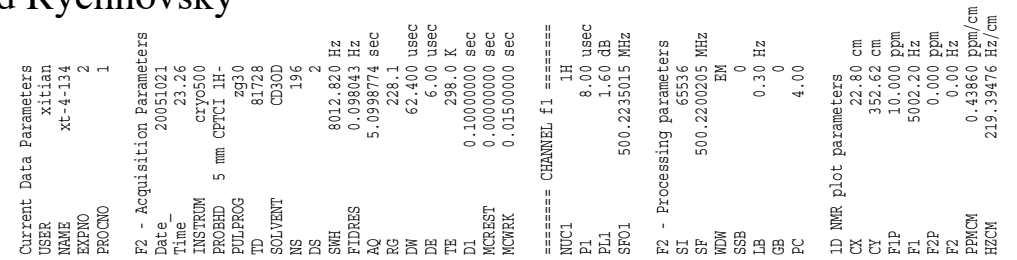

โ5062' $\mathrm{T}$

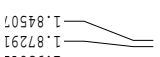

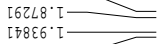

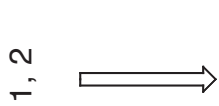

$27199 \cdot 2$
$10789 \cdot 2$

19969.5

э6รt8:

$366 \varepsilon 0^{\circ} \varepsilon$

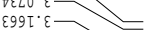

$969 \mathrm{I}^{\circ} \varepsilon \longrightarrow$

LZLT: $\varepsilon$

उซgor $\varepsilon$

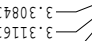

उLATE' $\varepsilon$

$9977 \cdot \varepsilon$

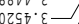

(1)

$66067 \cdot \varepsilon$

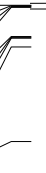

1)

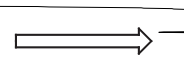

¿69 $\angle 8^{\circ} \sqcap-$

โ9TS6. $๐-$

โ2767 9

ะ8ร50.9

8 เ99.9-

$398 \angle 9 \cdot 9$

โ266L $30978 \cdot 9$

โ00E8.9

$28620^{\circ} \mathrm{L}$

${ }^{3} \operatorname{TEOZ}^{\circ} L$

ZL6T2:L

ETSTS $L=$

$\angle 8 T E S^{\circ} \mathrm{L}$

통

$687 L: L$

J9LI8: $\mathrm{C}$

บै

ชิ

嵒

III

-

究
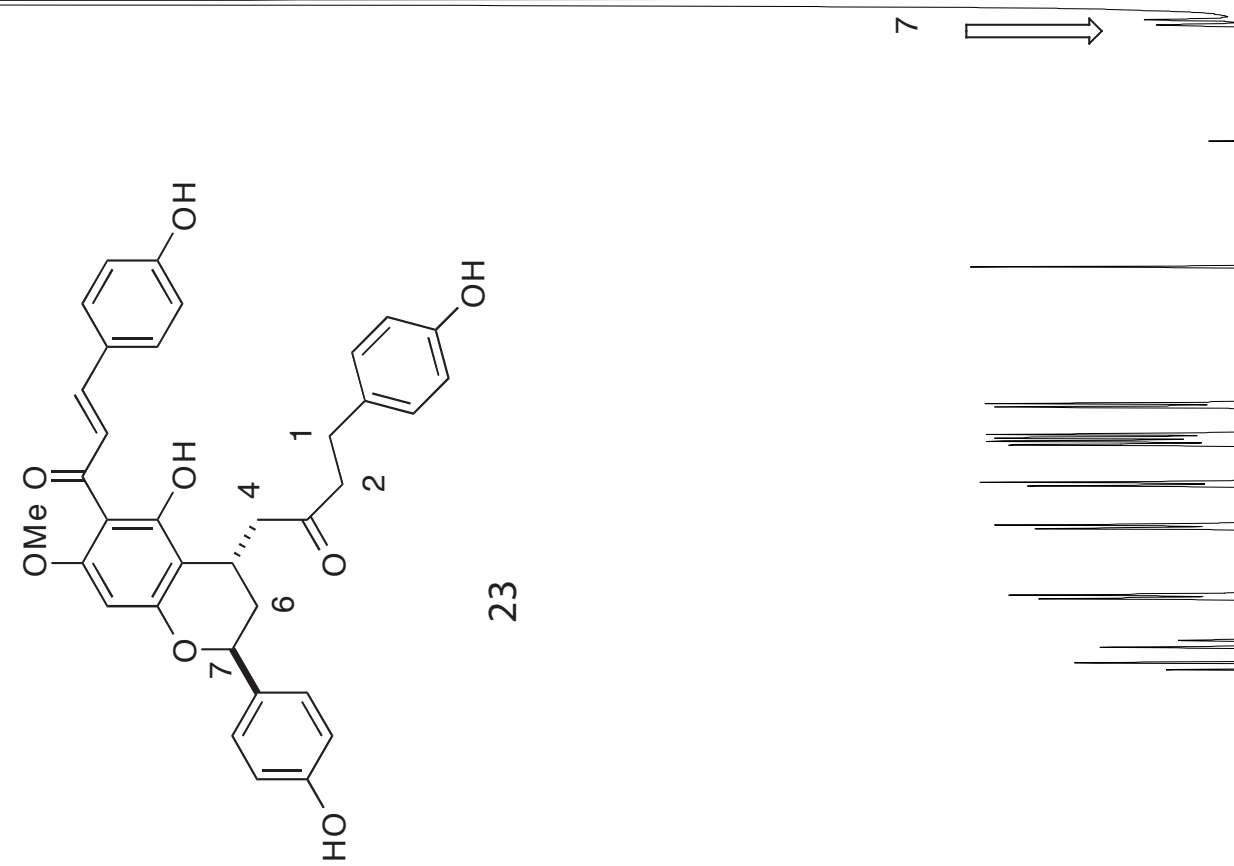
Tian, Jaber, and Rychnovsky

Page S-
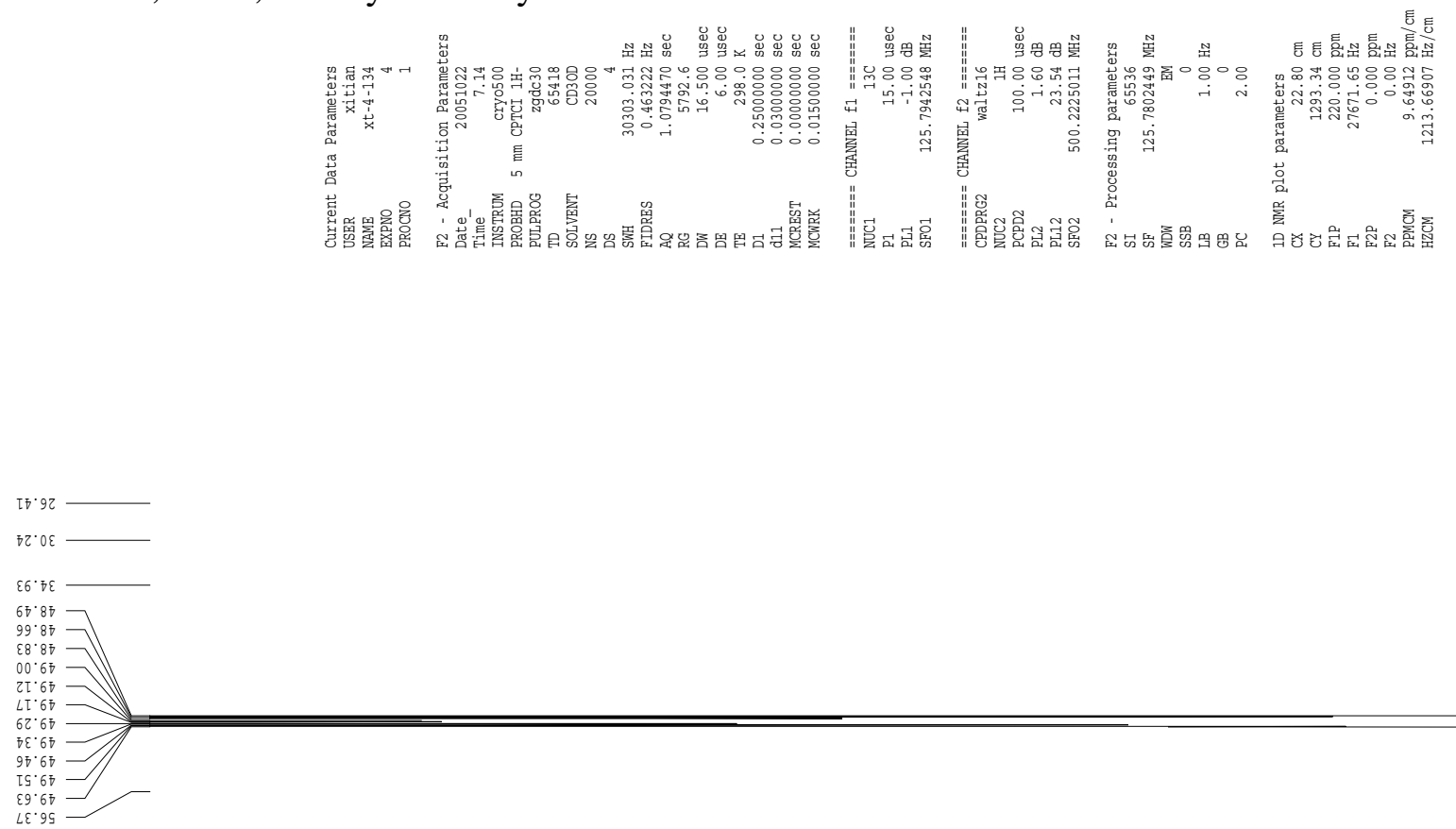

$09 \cdot 9 L$
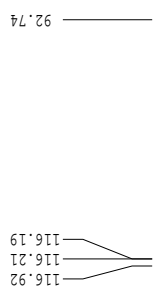

$\angle \varepsilon^{\circ} \varsigma Z T-$

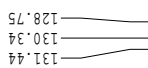

$D^{\prime} T^{\circ} \overline{D T}$

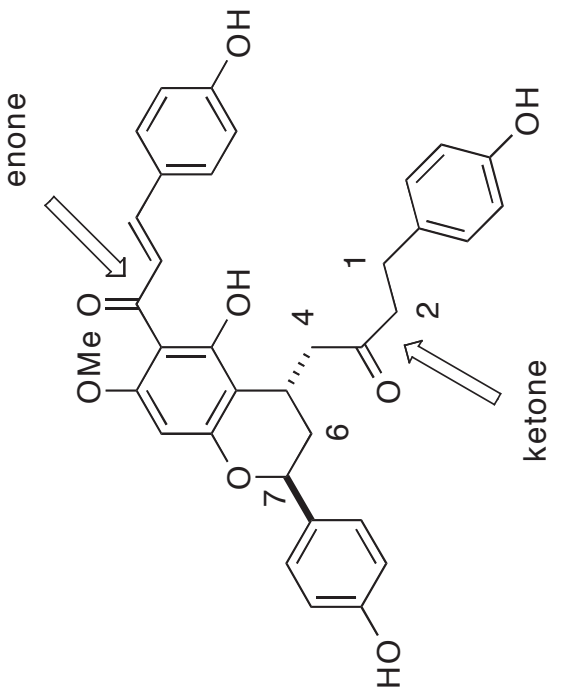

$\stackrel{m}{\sim}$
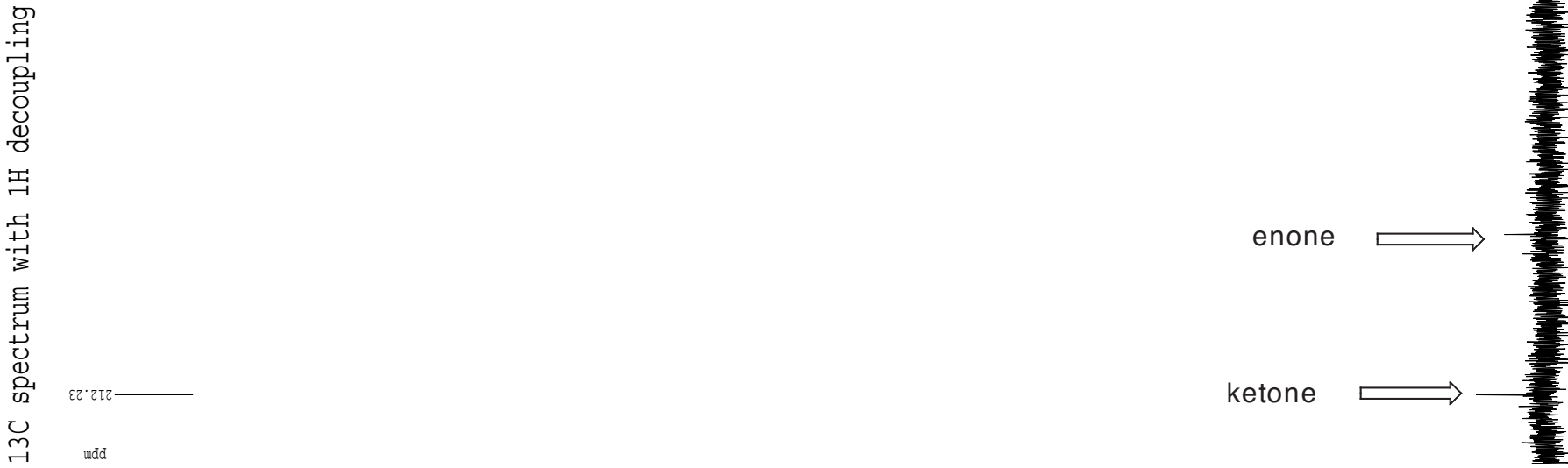
Tian, Jaber, and Rychnovsky

Page S-
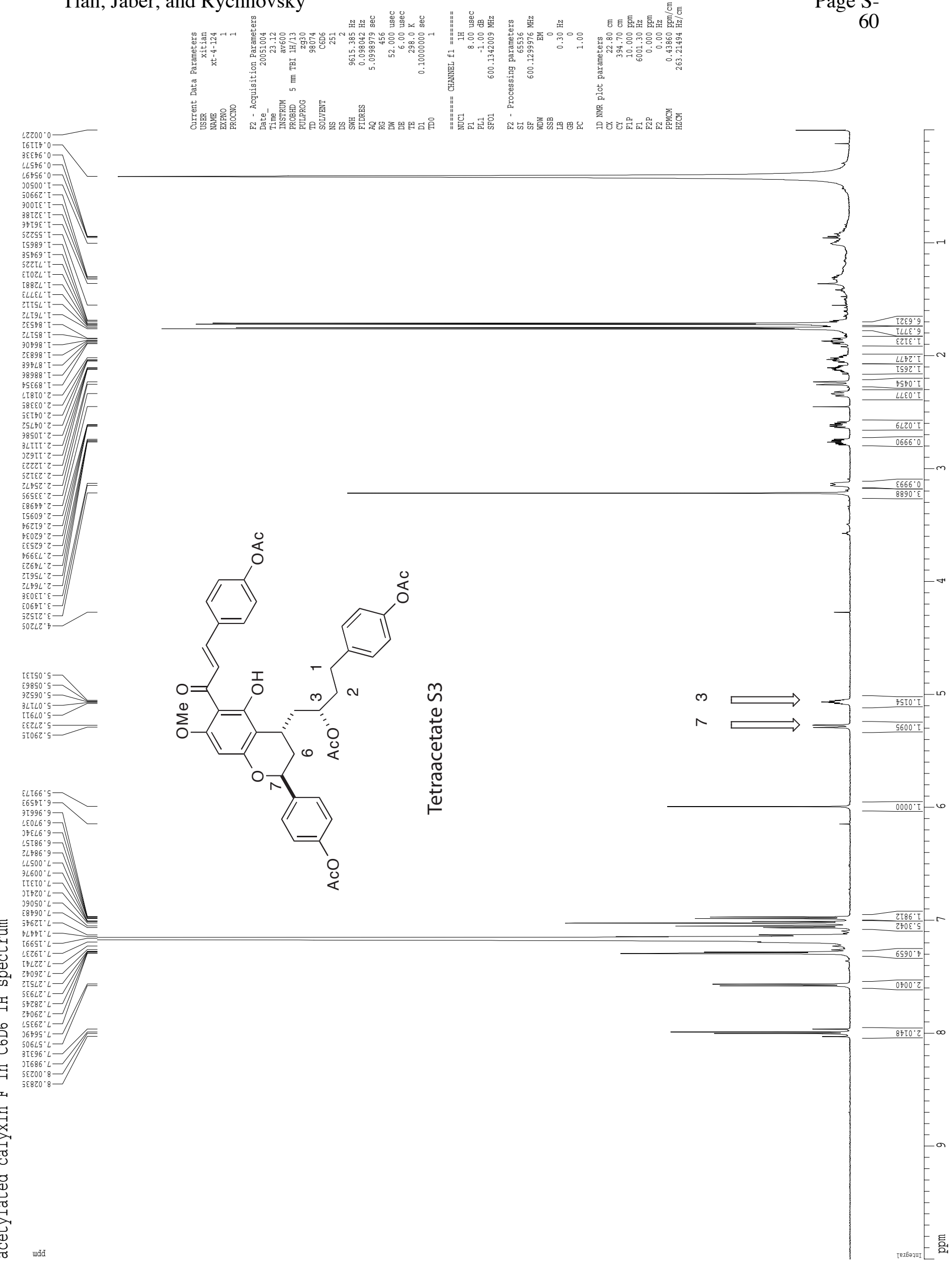
Tian, Jaber, and Rychnovsky
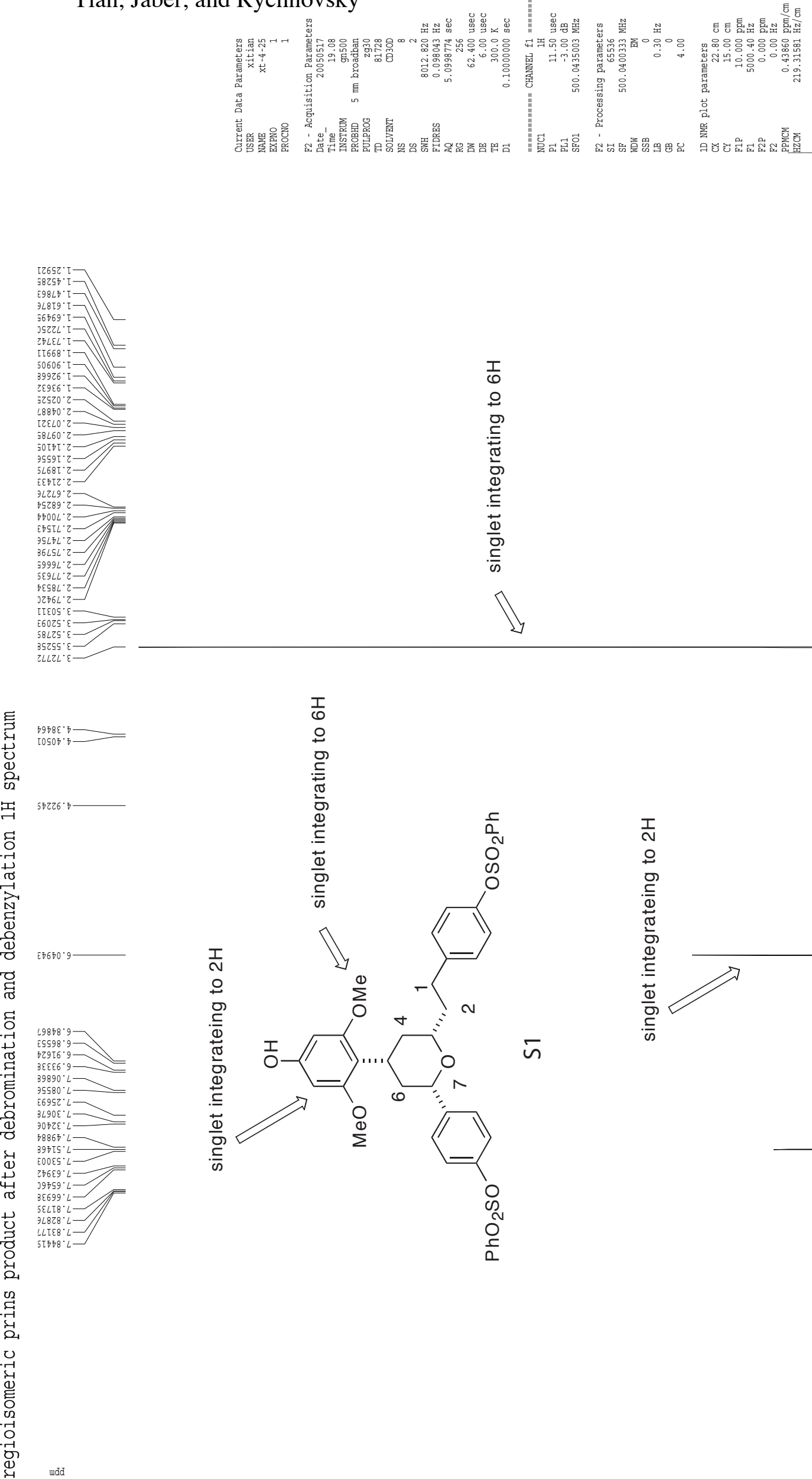

Page S-

61

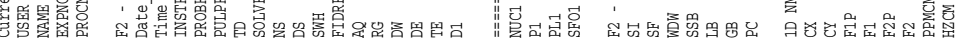


Tian, Jaber, and Rychnovsky

Page S-
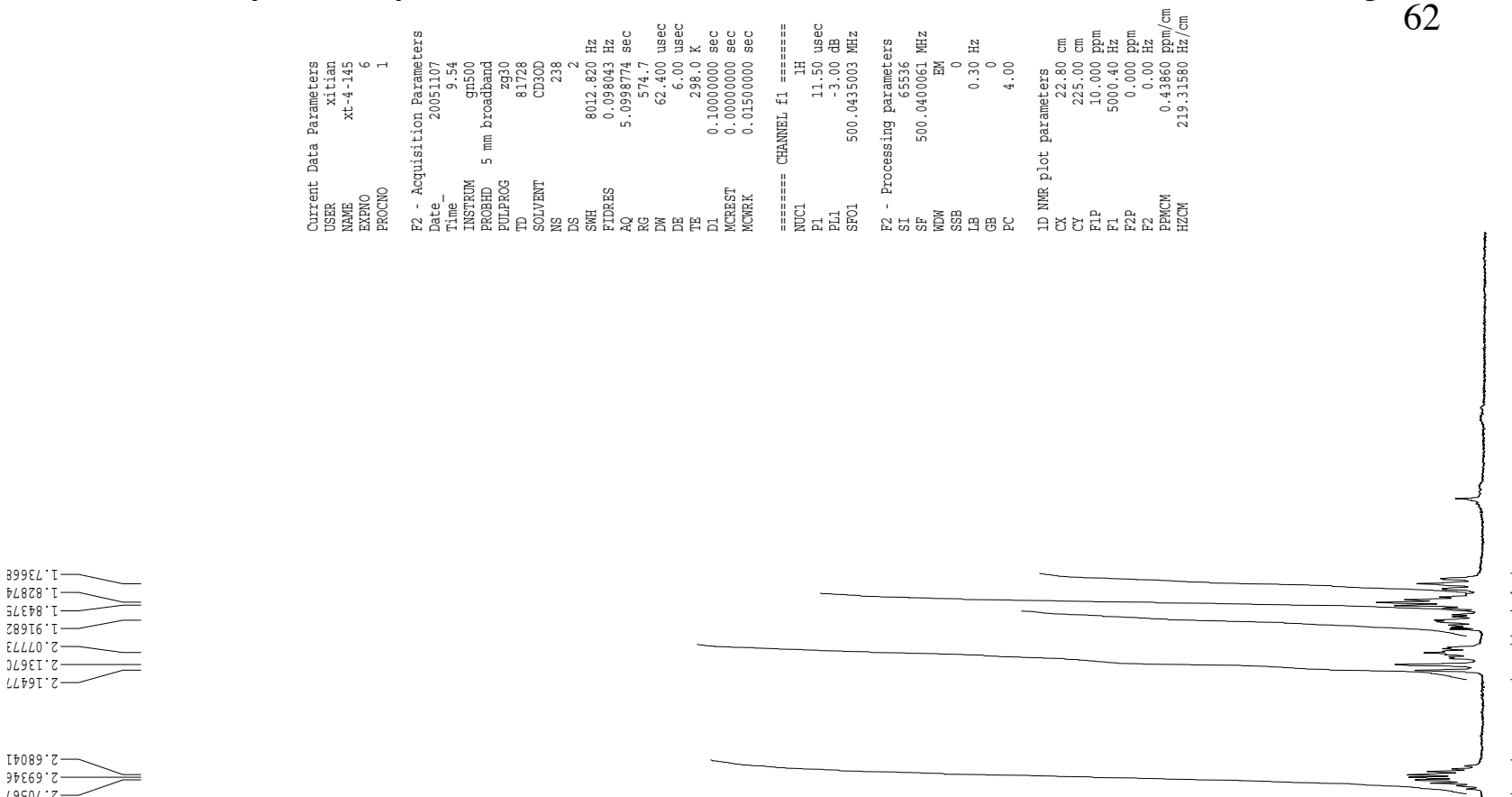

$\nabla \angle \sigma 6 \mathrm{~T} \cdot \varepsilon \longrightarrow$

$\angle 8 Z \varepsilon \tau^{\circ} \varepsilon \square$

$89 \varepsilon \varepsilon \cdot \varepsilon$

¿ $\angle 6 \varepsilon \varepsilon \cdot \varepsilon$

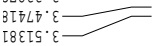

$\nabla \varepsilon 9 L L \cdot \varepsilon-$

ร86ร $\sigma^{\circ} \varepsilon$

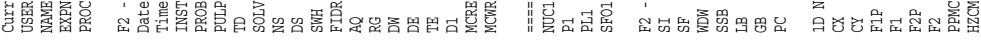

[8698.

$39850.9=$

$50 \angle L 0.9$

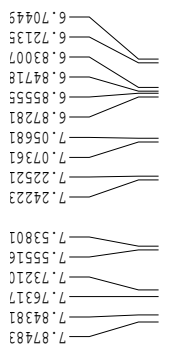

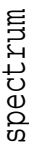
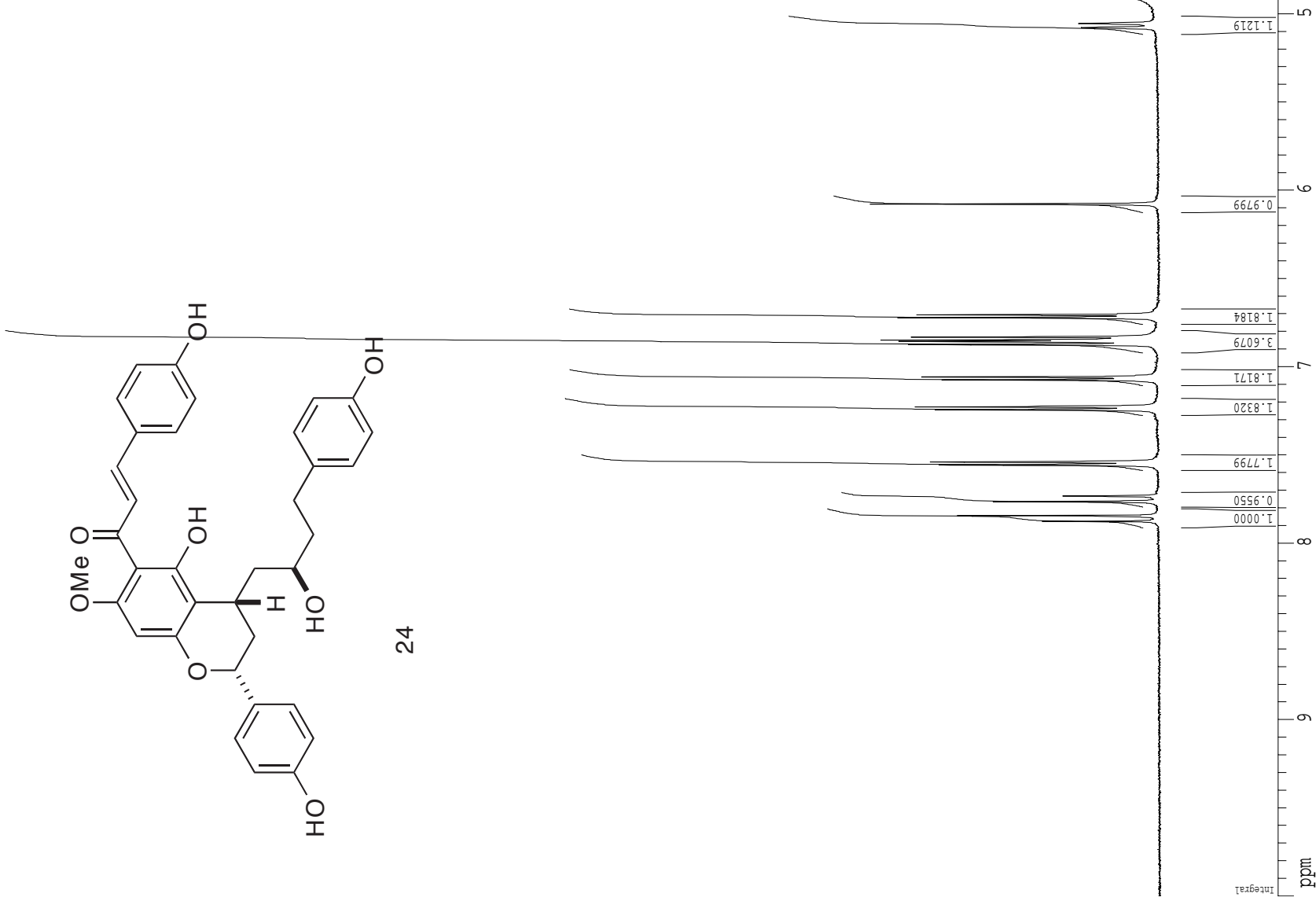
Tian, Jaber, and Rychnovsky

Page S-
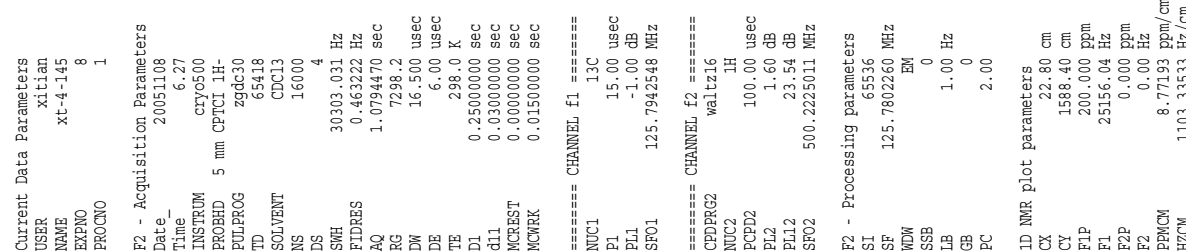

63

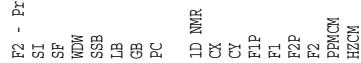

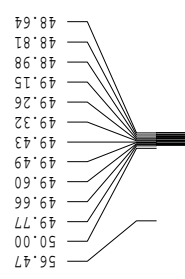

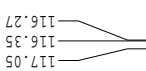

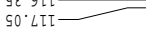

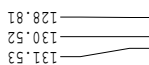

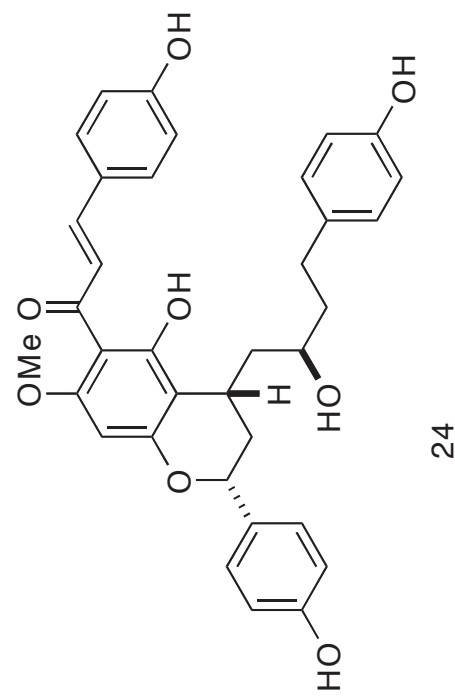

$\stackrel{+}{\sim}$

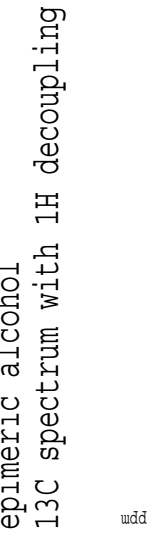

